\title{
Resonant Response in Bounded Nonlinear Wave Systems
}

\author{
by \\ Taqi Aldin Mohammed Shatnawi
}

Thesis Submitted to the Faculty of Graduate and Postdoctoral Studies

In partial fulfilment of the requirements for the degree of Doctor of Philosophy in Mathematics

School of Mathematics and Statistics

Faculty of Science

Carleton University

(C) Taqi Aldin Mohammed Shatnawi, Ottawa, Canada, 2017 


\section{Abstract}

In this thesis, we study the response of nonlinear wave systems in bounded domains at or near resonance. Such circumstances arise in numerous physical regimes. In particular, we examine the case of acoustic waves in various geometric configurations, the further understanding of which serves as a primary motivation for this work. There are typically two qualitatively distinct types of response that may be observed: continuous and shocked.

The main goal of this work is to understand the transition between these two regimes. To achieve this aim, we introduce a variety of nonlinear model problems at or near resonance and study the subsequent response. Then we explain how the features of this problem, such as the nonlinearity form and the nature of spectrum, play a fundamental role in the response.

We aim to demonstrate an essential condition for the existence of the unbounded solution and discuss the methodology for approximating the solution in that regime. Furthermore, we analyze the interaction between the spectrum and the nonlinear features of the model problem. There is a correlation found between a continuous response and incommensurate eigenvalues and between an unbounded response and commensurate eigenvalues. We carried out numerical simulations for each model problem with variation some features, such as the nonlinearity and boundary conditions, to study how this affects the outcome and the spectrum. The results of the numerical solutions are in a good quantitative agreement with the leading-order 
approximations.

Moreover, we present wave-model problems incorporating a damping effect to investigate how this affects the qualitative nature of the response. However, for some cases of the damped model problem, multiple modes must be included to improve the comparison between the analytical solution and the numerical results. We carry out a weakly nonlinear analysis of higher modes. In order to better understand the role of higher modes, we provide amplitude-frequency comparisons.

Our results provide a better understanding for the transition between the two responses through the simple model problems, and this in turn will contribute to providing new insights into more relevant problems in acoustic and other related regimes. 


\section{Acknowledgements}

First and foremost, I thank ALLAH (the God) for blessing me with many precious gifts.

It is my pleasure to express my deepest sincere gratitude to my supervisor, Professor David E. Amundsen for his guidance, help, advice and generous encouragement. I very much appreciate giving me the chance to work on this interesting research. His energy and enthusiasm have been a constant source of motivation for me. You will be my ideal model to follow in my coming academic life.

I cannot imagine how I express my thanks in words alone to my parents. I will start with my deepest pleasure to the man, who is the source of inspiration, without your infinite support and encouragement, this work cannot be done; to my father, you deserve this success more than me. To greatest woman in the world, to the sweet flower in the life, my mother, for her constant help and support during all my life, especially during my $\mathrm{PhD}$ studying.

My sincerest thanks go to my brothers and sisters, Eng. Ahmad, Eng. Raid, Taqwa, Raedh and Eng. Alaa for their love, support and infinite encouragement during my study.

I thank the staff, graduate students, and the faculty members at the School of Mathematics and Statistics for their friendship. I also thank the Hashemite University (Jordan) for providing me with financial assistance. 
Last, but not least, my warmest thanks to my wife Maysaloun for her support, encouragement and patient during the preparation of this thesis. Many special thanks go to my life sweet gift, my sons, Mohammed and Ibrahim, for the joy and happiness they provide to me during my studying. They have taught me the true meaning of life, both of whom I love so very dearly. 


\section{Dedication}

To my parents:

Mohammed Shatnawi \& Wasfieh Ramzi 


\section{List of Symbols}

- $\rho$ density

- P Pressure

- $\omega$ frequency

- M mach number

- $e$ condensation

- $r_{1}$ external boundary

- $r_{2}$ interior boundary

- A Arbitrary amplitude

- $\alpha(x, T)$ the nonlinear characteristic variable associated with outward waves

- $\chi$ small amplitude parameter

- $T(x, \alpha)$ the arrival time of the outward wave at location $x$

- $T(x, \beta)$ the arrival time of inward wave at location $x$

- $s_{1}$ the shock position

- $\alpha$ boundary condition variable 
- $\mu$ damping parameter

- $\phi(x)$ eigenfunction

- $\lambda_{n}$ higher mode of eigenvalue

- $A_{-} A[1]$ Analytic amplitude with using one mode

- $A_{-} A[2]$ Analytic amplitude with using two modes

- $A-\delta$ amplitude-frequency

- $\Re(\lambda)$ real part of eigenvalue $\lambda$

- $L$ spherical geometry parameter

- $k$ density variable parameter 


\section{List of Figures}

2.1 Differential volume element. . . . . . . . . . . . . . . . . 11

2.2 Spherical geometry. . . . . . . . . . . . . . . . . 24

2.3 Figure 1 from Amundsen [57] illustrating the comparison of numerical solution (solid) and geometric acoustic approximation (dashed) for gas in spherical shell $L=30, M=0.005$ for $u(0.5, \theta)$. . . . . . . . . . .

3.1 Plot of $u(x, \theta)$ as a function of $\theta$ where $\alpha=0$ at $x=0.5$. . . . . .

3.2 Comparison of numerical solution (solid line) to leading-order approximation (dashed line) $m=0.0001$ with $\alpha=2$, and the solution of (3.29)

3.3 Plot of $u(x, \theta)$ as a function of $\theta$ for Case 3 at $x=0.5 . \quad \ldots . . . \quad 48$

3.4 Plot of $u(x, \theta)$ as a function of $\theta$ for case 1 at $x=0.5 \ldots . . . . \quad 52$

3.5 Comparison of numerical solution (solid line) to leading-order approx-

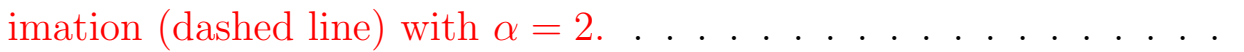

3.6 Plots solution of model problem $(3.5), u(x, \theta)$ as a function of $\theta$ with different form of nonlinearity with $\alpha=0$ at $x=0.5$. In (a) it is shown the result for $f(u)=u^{4} u_{x}$ while in (b) it is shown those obtained for $f(u)=u^{5} u_{x} \ldots \ldots \ldots \ldots \ldots \ldots \ldots \ldots$

3.7 Plot of $u(x, \theta)$ as a function of $\theta$ for Case 1 and the continuous response

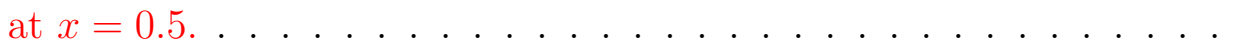

3.8 Comparison of numerical solution (solid line) to leading-order approximation (dashed line) with $\alpha=2$, and the solution of (3.42). . . . . 
3.9 Plot of $u(x, \theta)$ as a function of $\theta$ for Case 3 showing a continuous response at $x=0.5 \ldots \ldots \ldots \ldots$

3.10 Plots of the solution of model problem $(3.1), u(x, \theta)$ as a function of $\theta$ with nonlinear derivatives at $x=0.5$. In (a) it is shown the result for $f(u)=u_{x}^{2}$ while in (b) it is shown those obtained for $f(u)=u_{x}^{3}$. . .

3.11 Plots of the solution of model problem $(3.1), u(x, \theta)$ as a function of $\theta$ with trigonometric nonlinearity at $x=0.5$. In (a) it is shown the result for $f(u)=\sin (u)$ while in (b) it is shown those obtained for $f(u)=\cos (u) \ldots \ldots \ldots \ldots \ldots$

3.12 Plots of the solution of model problem $(3.1), u(x, \theta)$ as a function of $\theta$ with trigonometric and derivative nonlinearity terms at $x=0.5$. In (a) it is shown the result for $f(u)=\cos (u) u_{x}$ while in (b) it is shown those obtained for $f(u)=\sin (u) u_{x}$. . . . . . . . . . . . .

3.13 Effect of nonlinearity parameter $\beta$ on the transition between unbounded and continuous solutions for the model problem (3.3), the result for $f(u)=(1-\beta) u^{2}+\beta u u_{x}$, and $\beta=0.4,0.6,0.8(\mathrm{a}-\mathrm{c}) \ldots . . .$.

4.1 Comparison of numerical solution (solid line) to leading-order approximation (dashed line) with $\alpha=0.5 \ldots \ldots . . . \ldots$

4.2 Comparison of numerical solution (solid line) to single-mode leadingorder approximation (dashed line) with $\alpha=0.95 . \quad \ldots . . . . .977$

4.3 Comparison of numerical solution (solid line) to leading-order approximation (dashed line) with $\alpha=1 \ldots \ldots \ldots$

4.4 Comparison of the two-mode leading-order approximation solution (dashed line) and numerical solution (solid line) and single-mode (dotted line) with $\alpha=0.95 \ldots \ldots \ldots$

4.5 Comparison of numerical solution (solid line) to two-modes leadingorder approximation (dashed line) and one-mode (dotted line) with $\alpha=1 \ldots \ldots \ldots \ldots \ldots \ldots \ldots$ 
4.6 Comparison of numerical solution (solid line) to leading-order approximation with one mode (dashed line) with $\alpha=0.9999 . \quad$. . . . . . . 91

4.7 Comparison of numerical solution (solid line) to leading-order approximation with two-modes (dashed line) with $\alpha=0.9999 . . . . . . .991$

4.8 Comparison of numerical solution (solid line) to leading-order approximation with one mode (dashed line) with $\alpha=0.6$. . . . . . . . . . 92

4.9 Comparison of numerical solution (solid line) to leading-order approximation with two-modes (dashed line) with $\alpha=0.6 . . . . .$.

4.10 Comparison of amplitude curves from numerical solution (solid line) and the leading-order approximation using two modes (dashed line) and using single mode (dotted line). . . . . . . . . . . . . . .

4.11 The amplitude-frequency curves from numerical solution (solid line) and the leading-order approximation with two modes (dashed line) and single mode (dotted line) when $\alpha=1 . \quad$. . . . . . . . . . . 95

4.12 Comparison of one-mode approximation solution (dashed line) with $\delta=0$ to numerical solution (solid line) at resonance. . . . . . . . . . . 107

4.13 Truncation $N(\alpha)$ based on truncation parameter $c=1 . \quad$. . . . . 109

4.14 Comparison of numerical solution (solid line) to leading-order approximation (dashed line) $v 1(x, \theta)$ with $\alpha=0.95 \ldots . . . . . . . . .115$ 


\section{List of Tables}

3.1 Performance summary of the nonlinear types of model problem (3.5). $\quad 55$

3.2 Performances summary of the nonlinear types of model problem (3.1). $\quad 60$

3.3 Performance summary of the nonlinear types of model problem (3.1) with $f(u)=(1-\beta) u^{2}+\beta u u_{x} \ldots \ldots \ldots 65$

4.1 Summary of real eigenvalues of the Jacobian matrix in (4.71) . . . . . 102 


\section{Contents}

Abstract

Acknowledgements

Dedication vi vi vis

List of Symbols vii

List of Figures $\quad$ ix

List of Tables xii

1 Introduction and Literature Review 1

2 Mathematical Background $\quad 7$

2.1 Equations of motion . . . . . . . . . . . . . . . . . . 7

2.1.1 Descriptions of Fluid Motion . . . . . . . . . . . 8

2.1.2 Conservation of mass . . . . . . . . . . . . . . . 10

2.1.3 Conservation of momentum . . . . . . . . . . . . . . . 12

2.2 Perturbation Methods . . . . . . . . . . . . . . 15

2.2.1 Expansion Method ................ 16

2.2.2 Degenerate Perturbation Cases . . . . . . . . . . . . 17

2.3 Continuous Wave Motion . . . . . . . . . . . . . . . . . . . 23 
2.3.1 Resonant oscillations of an inhomogeneous gas between concen-

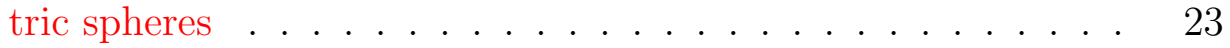

2.4 Shocked Wave Motion . . . . . . . . . . . . . . . . . . 31

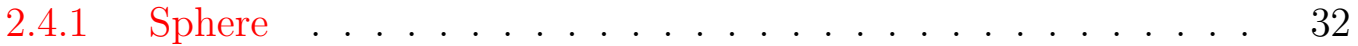

3 The Transition between Continuous and Unbounded Regimes 38

3.1 Formulation of Nonlinear model problems . . . . . . . . . . . . . 39

3.1.1 Nonlinear form $f(u)=u^{n} u_{x}$. . . . . . . . . . . . . . 40

3.1.2 The generalized result for $f(u)=u^{n} u_{x} \ldots \ldots . . . . . .53$

3.2 Nonlinearity Form $f(u)=u^{2} \ldots \ldots$. . . . . . . . . 56

3.3 Other Nonlinear Forms . . . . . . . . . . . . . . . 59

3.4 Summary ............................. 64

4 Nonlinear Wave Resonance with Damping $\quad 67$

4.1 Formulation of the Model Problem . . . . . . . . . . . 68

4.2 Analytic Approximation Using a Single Mode . . . . . . . . . . . . 68

4.3 Numerical Results and Comparison with Single Mode . . . . . . . . 74

4.4 Weakly Nonlinear Analysis with Higher Modes . . . . . . . . . . . . 79

4.5 Numerical Simulation and Comparison for Two Modes . . . . . . . . 86

4.6 Comparison of Amplitude . . . . . . . . . . . . . . . . . 93

4.7 Stability Analysis and the Effect of Damping . . . . . . . . . 96

4.8 The Case $\alpha=0$. . . . . . . . . . . . . . . . . . . . . . . . . . . . . 103

4.8.1 Formulation and linear theory . . . . . . . . . . 103

4.8.2 Nonlinear Theory: Amplitude-frequency Relation . . . . . . 105

4.8.3 Numerical solutions and comparison results . . . . . . . . . 106

4.9 Truncation of Modes $N(\alpha) \ldots \ldots \ldots$. . . . . . . . . 107

4.10 New Damped Model Problem . . . . . . . . . . . . . . . . . . 109

4.10 .1 The Model Formulation . . . . . . . . . . . . . . . 110

4.10.2 Analytic Approximation . . . . . . . . . . . . . . . 110 
5 Conclusions and Future Work 116

5.1 Conclusions . . . . . . . . . . . . . . . . 117

5.2 Future Directions . . . . . . . . . . . . . . . . . . . 118

$\begin{array}{ll}\text { A The Fredholm Alternative Theorem } & 121\end{array}$

$\begin{array}{lr}\text { Bibliography } & 124\end{array}$ 


\section{Chapter 1}

\section{Introduction and Literature Review}

The description of waves in many fields like acoustics and fluid dynamics may be captured by variants of so-called nonlinear wave equations. In particular, the study of acoustic resonant oscillations of an inhomogeneous gas contained in various geometries has been widely discussed both experimentally and theoretically. In 1939, Lettau [36] studied a closed straight cylindrical tube. His experiments were focused on the forced nonlinear, resonant response of gas in a container. He showed that, for small piston velocities, shock waves traverse the tube. On the other hand, Betchov [2] followed by Chu and Ying[5], Gorkov [22] and Chester[6, 7] introduced a theoretical explanation of the phenomenon, which in turn helped, for example, to provide a better understanding of noise excitation in jets and reciprocating engines.

Chester [8] studied the resonant oscillations of gas, contained in a vibrating spherical shell where the waves have spherical symmetry. He showed that the shock waves do not feature in the solution, and the technique that he used is called a dominant first harmonic approximation. In another study, Ellermeier [18] investigated the effect of cylindrical geometry on the resonant motion of a homogeneous gas and pointed out 
that it could result in shockless oscillations. For more details see [32, 33, 45]

Lawrenson et al. [35] observed a new phenomenon during some of their experiments. They showed that very high shockless pressures could be generated by resonant acoustic oscillations in specially shaped containers like a cone, horn or bulb. They called this Resonant Macrosonic Synthesis (RMS).

RMS was investigated numerically by Ilinskii et al. in 1998 [24], and they reproduced the amplitude-frequency curve of the experiments of Lawrenson qualitatively. Further numerical study was carried out by Chun and Kim in 2000 [11]. They obtained another numerical solution of the one-dimensional nonlinear equations for resonant oscillations when a viscosity term is included. They found that a bulb shape induced higher compression ratios than other shapes. In 2004, Mortell and Seymour [44] explained the phenomenon of RMS analytically by using the dominant first harmonic technique to obtain the solution, and they found a good qualitative agreement with experimental results.

In 1997, Ellermeier et al. [19] studied the resonant nonlinear oscillations of a gas in a cylindrical or spherical shell, using a similar potential formulation as Chester (1991) [8]. They showed that the difference between behaviour with respect to the planewave system is related to the linear eigenvalue spectrum. For the system supporting plane waves, the eigenvalue spectrum is commensurate; where higher eigenvalues correspond to integer multiples of the fundamental frequency, and this is not so for the cylindrical or spherical case.

Subsequently, Mortell et al. in 2009 [43] theoretically investigated the resonant macrosonic waves in cones and bulbs, where the resonance frequency is incommensurate, and found that the responses are shockless. They used a similar analysis scheme as in Mortell and Seymour (2004) [44], which involves introducing a new dependent variable. They determined that the theoretical solutions agree with the experimental results in Lawrenson et al. [35]. 
Seymour et al. [56] studied the resonant oscillation of an inhomogeneous gas between two concentric spheres when the response is continuous by using a dominant firstharmonic approximation. They explained how the effect of the geometrical parameter and the Mach number could play a significant role in the qualitative behavior of the outcome. Seymour et al. found that the continuous responses could be changed to a shocked response.

Subsequently, Seymour et al. [57] conducted research to describe shocked resonant oscillations between concentric spheres and coaxial cylinders when the dominant firstharmonic approximation is not valid. They explained how a nonlinear geometric acoustics approximation could predict shocked flows. A primary feature of this work was to understand how the existence of shocked solutions was shape dependent.

The previous results showed that acoustic wave problems may result in the formation of shocks, but many parameters may affect the behaviour of the outcome, and transitions to continuous response exist. It can be noted that the effects of the geometry, nonlinearity and stratification parameters are crucial to the qualitative nature of the resonant oscillation.

Recently Amundsen et al. [15] investigated the effect of the density parameter in the resonant oscillation of a gas contained in the frustum of a cone. In order to study the continuous resonant solution, they constructed a variable density profile depending on an arbitrary parameter to approximate a desired density, then used it to reflect the eigenvalue equation corresponding to the linear theory. For shocked response, they used Whitham technique that depends on approximating the motion by oppositely travelling simple waves.

There are two main goals of this thesis. First, we provide a better understanding of the transition between the continuous and unbounded resonant response regimes. In doing so, various nonlinear model problems will be studied. This will provide a simple setting in which to explain how features such as geometry, eigenvalue spectrum and 
nonlinearity play a significant role in the nature of outcomes. We will also investigate the detailed nature of the transition between the two regimes. Finally, we will explain the correlation between the qualitative nature of the response and the commensurate or incommensurate nature of the underlying linear spectrum.

Second, we will study how the inclusion of damping into our nonlinear model problem will affect the transition between the continuous and unbounded responses. To achieve this goal, we obtain an analytic approximation for various model problems with damping included, and we also compare with the numerical solutions. In this part of the thesis, we will explain how the higher modes in the analytical approach must be included to provide a valid comparison between the numerical solution and analytical approximation. Finally, we provide amplitude - frequency comparisons to obtain a more complete understanding of the effect of inclusion of higher modes.

An overview of the work in this thesis is as follows. Chapter 2 will briefly present the equations of motion, conservation laws of mass and momentum, which form the fundamental equations of fluid dynamics. It will also describe the main mathematical tools utilized to help understand acoustic oscillations and unbounded solutions at or near resonance. We then introduce the two resonant response regimes: continuous and shocked. As an example, we describe the resonant oscillation of an inhomogeneous gas between two concentric spheres. This part investigates the effect of the geometry and how the motion can change from continuous to shocked. The relation between the eigenvalues and response will be explained through investigating some asymptotic forms of the eigenvalue equation. At the end of this part, we discuss how to recover some previous results for simpler geometries such as a full sphere and a straight tube. Finally, we explore when the first harmonic approximation breaks down and we need to use another method, like nonlinear geometric acoustic analysis, to approximate the shocked motion.

In chapter 3 , the transition between the continuous and unbounded resonant response 
in an undamped model nonlinear wave system is discussed. To explain the transition, some features that affect the existence of the shock solution, like the form of the nonlinearity in the model problem, will be discussed. In this part of the thesis, we will discuss how the form of the nonlinear term can affect the amplitude condition in the leading-order solution and where the resonance could occur. We will explain this by introducing nonlinear model problems and study a variety cases of the nonlinear term. We will then also investigate a particular class of nonlinear model problems in which the velocity $u$ has the form $u^{n} u_{x}$. The results are summarized in tables 3.2 and 3.3 for this type of model problem.

Chapter 4 is dedicated to a damped nonlinear model problem where a weakly nonlinear analytic solution is studied to determine the behaviour of the approximate solution for the damped model problem under a variation of the boundary condition. The solvability conditions for a variety of configurations will be obtained to provide comparisons between analytical approximations and numerical solutions. We notice that the comparison between the approximate solution using a single mode in the leading-order with the numerical solution does not agree well in some cases. The analytic solution will be augmented to include multiple modes in the leading-order solution. That will in turn help to improve the comparison of the results. Then the numerical simulation will be carried out for the damped nonlinear model problem. However, to clarify the nature of the transition between a single mode in the linear theory of the analytical approximation solution (i.e., the leading-order $u_{1}(x, \theta)$ ) and inclusion of multiple modes, we present two additional comparisons: one for amplitudes and another for amplitude - frequency curves. Also, we discuss how the relation between the boundary conditions and forcing of the nonlinear model problem will play a main role in rescaling the perturbation expansion of the analytic solution. Next, the stability for some cases will be studied by using multiple scale methods, as well as the eigenvalues of the Jacobian matrix.

Finally, in Chapter 5, we summarize and conclude with some unanswered questions 
that arise in this study and that are left for future research. Then we finish the thesis with some appendices that contain some basic concepts and background knowledge needed in various parts. 


\section{Chapter 2}

\section{Mathematical Background}

This chapter aims to briefly introduce the equations of motion and conservation laws, and it will describe the main mathematical tools we use in this thesis to help understand acoustic oscillations near resonance. See, for example [17, 38, 52, 54, 64]. In Section 2.1, we discuss the conservation laws of mass and momentum, which lead to the equations of fluid motion known as the Euler equations. It also includes some ways to describe fluid motion. Section 2.2 describes the key ideas of perturbation analysis, which underlie the analytic techniques used in later chapters. For more complete description of these concepts refer to [ [47]-[50], [1]]. Sections 2.3 and 2.4 will explain the two generic types of response that usually arise in resonant acoustic oscillations. The transition between these regimes is a key focus and will be studied later in this thesis.

\subsection{Equations of motion}

Some basic knowledge about the main equations of fluid dynamics is needed to study acoustic waves. These equations are presented in this section, including conservation of mass and momentum, which together comprise the Euler equations that appear in 
the last part.

\subsubsection{Descriptions of Fluid Motion}

To describe the fluid motion, one needs to know the variation of physical quantities, such as density, velocity and pressure, as functions of time. There are two ways to describe fluid motion. One is called Lagrangian, where one follows all fluid particles and describes the variations around each fluid particle along its trajectory. This method resolves contact discontinuities, since they coincide with the Lagrangian coordinates. The other way is Eulerian, where the variations are described at all fixed points as a function of time and where different particles pass the same point at different times. The Eulerian description is relatively simple and is more amenable to numerical computation and analysis.

\section{Lagrangian description}

Suppose a particle is identified by its initial position at time $t_{0}$,

$$
\vec{x}_{0}=\left(x_{0}, y_{0}, z_{0}\right)
$$

Let the position of the same particle at the later time $t$ be

$$
\vec{x}=\vec{x}\left(\vec{x}_{0}, t\right)
$$

Consider the initial position $\vec{x}_{0}$ to be uniquely represented by quantities $a, b, c$ to identify the particle:

$$
\vec{x}_{0}=\vec{x}_{0}(\vec{a}) \quad \vec{a} \equiv(a, b, c)
$$

Thus the path of a particle identified by $\vec{a}$ is given by : $\vec{x}=\vec{x}(\vec{a}, t)$. 
Now the particle velocity is

$$
\vec{u}_{L}=\left.\frac{\partial \vec{x}}{\partial t}\right|_{\vec{a}},
$$

and the particle acceleration is

$$
\left.\frac{\partial \vec{u}_{L}}{\partial t}\right|_{\vec{a}}=\left.\frac{\partial^{2} \vec{x}}{\partial t^{2}}\right|_{\vec{a}} .
$$

Other physical quantities, such as density $\rho$ and pressure $P$, can also be expressed in terms of $\vec{a}$ and $t$; for example, the density $\rho=\rho(\vec{a}, t)$ and the pressure $P=P(\vec{a}, t)$.

\section{Eulerian description}

In this method, all variables are taken to be functions of time and local position, instead of initial position, where the local position is given by the position vector $\vec{x}$. Suppose first that the Lagrangian information of the velocity of all particles is known:

$$
\vec{u}_{L}=\vec{u}(\vec{a}, t) .
$$

Then, by the definition of velocity, we obtain

$$
\frac{\partial \vec{x}}{\partial t}=\vec{u}_{L}(\vec{a}, t) .
$$

After integrating, we get the position of a particle,

$$
\vec{x}=\int \vec{u}_{L} d t=\vec{x}(\vec{a}, t) .
$$

This result can be inverted in principle to get

$$
\vec{a}=\vec{a}(\vec{x}, t) .
$$


Once the position of a particle at time $t$ is known, any physical property of a particle can be associated to the property at a certain position in space. For example, from the Lagrangian pressure $P(\vec{a}, t)$, we get the Eulerian pressure $P(\vec{a}(\vec{x}, t), t)=P(\vec{x}, t)$.

Next suppose that the Eulerian velocity field is known everywhere: $\vec{u}=\vec{u}(\vec{x}, t)$. Since at time $t$ the particle starting at $\vec{a}$ is at $\vec{x}(\vec{a}, t)$, then the Eulerian and Lagrangian velocities of a particle $\vec{a}$ are the same. Therefore

$$
\vec{u}_{E}(\vec{x}, t)=\frac{\partial \vec{x}}{\partial t}
$$

If we solve for $\vec{x}=\vec{x}(\vec{a}, t)$, subject to the initial condition $\vec{x}=\vec{x}_{0}(\vec{a})$ and $t=t_{0}$, we then have:

$$
\frac{\partial \vec{x}}{\partial t}=\vec{u}_{E}[\vec{x}(\vec{a}, t), t] \equiv \vec{u}_{L}(\vec{a}, t)
$$

Conservation of mass has a Lagrangian form. However, only the Eulerian description is amenable when the fluid particles can exchange a quantity like a momentum as we will discuss in the coming subsections.

\subsubsection{Conservation of mass}

The conservation of mass law states that mass may neither be created nor destroyed. Therefore, it remains constant over time for a closed fluid system. This type of conservation can be expressed by an equation called the continuity equation, which plays an important role in many fields, especially in science and engineering. To derive this equation in Cartesian coordinates, consider the stationary differential volume of length $\Delta x$, width $\Delta y$ and height $\Delta z$ as illustrated in Figure 2.1, which shows a rectangular cube with three sides defined by three directed distances, $\Delta x, \Delta y$ and $\Delta z$. 


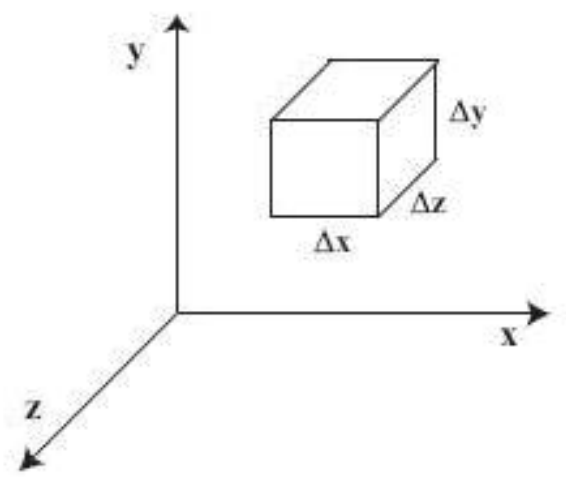

Figure 2.1: Differential volume element.

As the rate of change of the mass for this volume element $(\Delta V=\Delta x \Delta y \Delta z)$ is the difference between the rate of mass convected into and out of $\Delta V$. By assuming the fluid density within $\Delta V, \rho$ is continuous and differentiable, we have

$$
\begin{array}{r}
\Delta x \Delta y \Delta z \frac{\partial \rho}{\partial t}=\Delta y \Delta z\left[\left.\left(\rho v_{x}\right)\right|_{x}-\left.\left(\rho v_{x}\right)\right|_{x+\Delta x}\right]+ \\
\Delta x \Delta z\left[\left.\left(\rho v_{y}\right)\right|_{y}-\left.\left(\rho v_{y}\right)\right|_{y+\Delta y}\right]+\Delta x \Delta y\left[\left.\left(\rho v_{z}\right)\right|_{z}-\left.\left(\rho v_{z}\right)\right|_{z+\Delta z}\right]
\end{array}
$$

where $v=\left(v_{x}, v_{y}, v_{z}\right)$ is the velocity. By dividing both sides of equation (2.12) by $\Delta V$ and taking the limit as $\Delta V \rightarrow 0$, we obtain

$$
\frac{\partial \rho}{\partial t}=-\left[\frac{\partial}{\partial x}\left(\rho v_{x}\right)+\frac{\partial}{\partial y}\left(\rho v_{y}\right)+\frac{\partial}{\partial z}\left(\rho v_{z}\right)\right]
$$

This can be expressed more concisely as

$$
\frac{\partial \rho}{\partial t}=-\nabla \cdot(\rho \vec{v})
$$

This equation describes the differential form of conservation of mass and is called the continuity equation. By using the substantial derivative $\frac{D}{D t}$ (i.e., moving with the fluid particles the Lagrangian expression of the kinematics and dynamics of the flow 
field), equation (2.14) can be written as

$$
\frac{D \rho}{D t}=-\rho(\nabla \cdot \vec{v})
$$

where $\frac{D}{D t}=\frac{\partial}{\partial t}+\vec{v} \cdot \nabla$. Note that if the fluid density is constant, equation (2.15) reduces to

$$
\nabla \cdot \vec{v}=0
$$

\subsubsection{Conservation of momentum}

If a system does not interact with its environment in any way, certain properties of this system cannot change. Such quantities are referred to as conserved, and this leads to associated conservation laws. There are many examples of conserved quantities, such as mass (as discussed above), momentum and energy. The focus of discussion here will be on conservation of momentum.

Momentum $(\vec{p})$ is the product of the mass and the velocity of an object

$$
\vec{p}=m \vec{v}
$$

Using this quantity, we can rewrite Newton's second law as

$$
\vec{F}=m \vec{a}=m \frac{d \vec{v}}{d t}=\frac{d \vec{p}}{d t}
$$

assuming mass is constant.

Now we explain how the equation of motion for fluid flow is derived by using conservation of momentum and conservation of mass.

Suppose there is a fluid with local density $\rho(t, x, y, z)$ and local velocity $\vec{v}(t, x, y, z)$. Consider a control volume $V$ that has boundary $S$. Then the total momentum in the 
control volume without external forcing is

$$
\prod_{i}=\int_{V} \rho v_{i} d V
$$

where the index $i$ runs over the three components of the momentum. Therefore the rate of change of momentum is

$$
\frac{d \prod_{i}}{d t}=\int_{V} \frac{d\left(\rho v_{i}\right)}{d t} d V
$$

We assume there are no applied forces and that pressure and viscous forces are negligible. Then, the only way a momentum change can occur is by momentum flowing across the boundary:

$$
\frac{d \prod_{i}}{d t}=\int_{S}\left(\rho v_{i}\right) \vec{v} \cdot d S=\int_{S}\left(\rho v_{i}\right) v_{j} d_{j} S
$$

The surface integral can be changed into a volume integral using Green's theorem, to obtain

$$
\frac{d \prod_{i}}{d t}=-\int_{V} \nabla_{j}\left(\rho v_{i} v_{j}\right) d V
$$

By comparing equation (2.20) with equation (2.22), we can see that they are equal, no matter what volume $V$ is chosen, so the integrands must be pointwise equal. This gives an expression for the local conservation of momentum:

$$
-\nabla_{j}\left(\rho v_{i} v_{j}\right)=\frac{\partial\left(\rho v_{i}\right)}{\partial t} \cdot(2.23)
$$

Therefore, by using vector notation, the above equation can written as:

$$
\rho\left(\frac{\partial}{\partial t}+\vec{v} \cdot \nabla\right) \vec{v}=0
$$

Consider now the effect of pressure $P$. It contributes a force on the particles in the 
control volume, namely,

$$
F_{i}=\int P d_{i} S=-\int \nabla_{i} P d V
$$

A uniform gravitational field defined by a vector $\vec{g}$ contributes another force, namely,

$$
F_{i}=\int \rho g_{i} d V
$$

These forces contribute to changing the momentum, by Newton's second law:

$$
\frac{d \prod_{i}^{\prime}}{d t}=F_{i}
$$

where $\frac{d \prod_{i}^{\prime}}{d t}$ is the time rate change of momentum for each particle. Notice that we write $\frac{d}{d t}$ rather than $\frac{\partial}{\partial t}$ and $\prod_{i}^{\prime}$ rather than $\prod$ because Newton's laws apply to particles, not to the control volume. The rate of change of $\prod$, the momentum in the control volume, balances with the Newtonian contributions in equations (2.25) and (2.26) plus the flow contributions (2.23).

As explained in equation (2.15), by combining all the contributions, the equation of motion can be obtained:

$$
\frac{\partial\left(\rho v_{i}\right)}{\partial t}+\nabla_{j}\left(\rho v_{i} v_{j}\right)=-\nabla_{i} P+\rho g_{i}
$$

If we expand the left-hand side, we get:

$$
\rho \frac{\partial\left(v_{i}\right)}{\partial t}+v_{i} \frac{\partial(\rho)}{\partial t}+v_{i} \nabla_{j}\left(\rho v_{j}\right)+\rho v_{j} \nabla_{j}\left(v_{i}\right)=-\nabla_{i} P+\rho g_{i}
$$

where the second and third terms cancel by conservation of mass. Then we obtain:

$$
\rho \frac{\partial\left(v_{i}\right)}{\partial t}+\rho v_{j} \nabla_{j}\left(v_{i}\right)=-\nabla_{i} P+\rho g_{i}
$$


where $g_{i}$ is the ith component of the vector $\vec{g}=(0,0, g)$, and $g$ is the acceleration of gravity.

If we convert from component notation to vector notation, we get the common version

$$
\rho\left(\frac{\partial}{\partial t}+\vec{v} \cdot \nabla\right) \vec{v}=-\nabla P+\rho g .
$$

If viscous effects are negligible, then, by combining the momentum equation (2.31) with the conservation of mass equation (2.15), we obtain the Euler equations, which can be written as

$$
\begin{aligned}
\rho \frac{D \vec{v}}{D t} & =-\nabla P+\rho g \\
\frac{D \rho}{D t} & =-\rho(\nabla \cdot \vec{v}) .
\end{aligned}
$$

Finding the exact solution of these equations of fluid mechanics is not possible in all settings. However, some techniques for approximate computation of solutions can be used. In the next sections, we will explain an important class of these techniques: perturbation methods.

\section{$2.2 \quad$ Perturbation Methods}

In general for fluid and wave problems, the equations that model the system can be determined, but finding an exact solution (or an analytical solution) is very difficult. However, the solution can be often sufficiently approximated for our needs and within a certain error.

One set of common techniques for approximating solutions of differential equations is perturbation methods, which try to exploit small parameters in the system. If a small parameter can be identified, then perturbation methods may be applicable. Also, 
this technique can apply to many other classes of problems. Perturbation methods involve the expansion of the solution in terms of the small parameter. The terms in this expansion are then computed sequentially via the expansion method that will be explained briefly and exemplified below. We refer the reader to $[23,31]$ for more details.

\subsubsection{Expansion Method}

The main idea of this method is to expand the solution about one of the unperturbed roots in terms of unknown parameters and then substitute this expansion into the model equation and formally equate the powers of $\epsilon$ on both sides. Finally, this expansion is truncated after some number of terms to get an approximation.

Example : Consider the following quadratic equation for $x$ which involves the small parameter $\epsilon$ :

$$
x^{2}+\epsilon x-1=0,
$$

where $0<\epsilon \ll 1$.

Let

$$
x(\epsilon)=x_{0}+\epsilon x_{1}+\epsilon^{2} x_{2}+\epsilon^{3} x_{3}+\cdots,
$$

with $x_{0}, x_{1}, x_{2}, x_{3}, \cdots$ to be determined.

Substituting this expansion into equation (2.33), then equate the powers of $\epsilon$ on both sides of the equation to get:

$\epsilon^{0}: x_{0}^{2}-1=0 \Rightarrow x_{0}= \pm 1$, but for simplicity we only consider $x_{0}=1$.

$\epsilon^{1}: 2 x_{1}+1=0 \Rightarrow x_{1}=-\frac{1}{2}$.

$\epsilon^{2}: x_{1}^{2}+2 x_{2}+x_{1}=0 \Rightarrow x_{2}=\frac{1}{8}$. 
$\epsilon^{3}: 2 x_{3}+2 x_{1} x_{2}+x_{2}=0 \Rightarrow x_{3}=0$.

Therefore we obtain an approximate solution given by:

$$
x(\epsilon)=1-\frac{1}{2} \epsilon+\frac{1}{8} \epsilon^{2}+O\left(\epsilon^{4}\right),
$$

which is truncated after the third term. Similarly, if we let $x_{0}=-1$ we get an expansion for the other root. Notice that if we compare the expansion result with the exact solution of the quadratic equation (2.33) namely $x=-\frac{1}{2} \epsilon \pm \sqrt{1+\frac{1}{4} \epsilon^{2}}$, and when we expand the square root term up to $O\left(\epsilon^{4}\right)$, we get $x=-\frac{1}{2} \epsilon \pm\left(1+\frac{1}{8} \epsilon^{2}+O\left(\epsilon^{4}\right)\right)$ we see that the approximate solution agrees with the expansion of the exact solution. Notice that, to use the expansion method, it is necessary to assume that the result can be expanded in powers of $\epsilon$ and that the formal substitution and associated manipulations are permitted.

In some cases, the regular perturbation method illustrated above fails. In the following examples, we will illustrate some representative cases.

\subsubsection{Degenerate Perturbation Cases}

In this subsection, we present three examples to show how the regular perturbation may not be valid. The first example introduces a quadratic equation when the leading-order approximation provides only one root; this type of problem is called a singular perturbation problem. The second example explains that we need to capture the correct perturbation expansion, while the last example introduces Duffing's equation. 


\section{Example:}

Consider the quadratic equation

$$
\epsilon x^{2}+x-1=0
$$

and start with perturbation expansion:

$$
x=x_{0}+\epsilon x_{1}+\epsilon^{2} x_{2}+\epsilon^{3} x_{3}+\cdots .
$$

After substituting into equation (2.36) and grouping terms of $O(1), O(\epsilon)$, and $O\left(\epsilon^{2}\right)$ we obtain the solution

$$
x=1-\epsilon+2 \epsilon^{2}+O\left(\epsilon^{3}\right) .
$$

We obtain only one solution for the quadratic equation, as the second root is going to infinity as $\epsilon \rightarrow 0$. However, we can determine this root by looking for a two-term dominant balance in equation (2.36). Then, we get the consistent balance $x=O\left(\epsilon^{-1}\right)$. Therefore, we introduce a re-scaled variable $y$, where

$$
y=\epsilon x,
$$

where $y=O(1)$ as $\epsilon \rightarrow 0$.

We should notice that this re-scaling enables us to change the problem from a singular perturbation, in terms of $x$, into a regular perturbation, in terms of $y$.

After using a similar perturbation procedure as above in terms of $y$, the corresponding solutions for $x$ are

$$
x=\frac{-1}{\epsilon}-1+\epsilon .
$$


The perturbation expansion method is used in many problems to find approximate solutions, as shown in the previous example, but care needs to be taken to ensure the correct form of the expansion, as we will see in the next example.

\section{Example:}

Let us consider the solution of the equation

$$
x^{2}+(2+\epsilon) x+1=0 \text {. }
$$

Let

$$
x=x_{0}+\epsilon x_{1}+\epsilon^{2} x_{2}+\epsilon^{3} x_{3}+\cdots .
$$

Using the same technique, after collecting coefficients of like powers of $\epsilon$, we have leading-order terms

$$
x_{0}^{2}+2 x_{0}+1=0 \Rightarrow x_{0}=-1 \text {, }
$$

and the first correction is given by

$$
2 x_{0} x_{1}+2 x_{1}+x_{0}=0 \Rightarrow-1=0 \text {. }
$$

Therefore, we do not have a consistent perturbation expansion. Notice that equation (2.41) has the exact solution

$$
x=-1 \pm \sqrt{\epsilon}-\frac{\epsilon}{2} \pm \cdots
$$

Hence, we in fact should consider the perturbation expansion in powers of $\sqrt{\epsilon}$

$$
x=x_{0}+\sqrt{\epsilon} x_{1}+\epsilon x_{2}+\cdots,
$$

which will yield the correct solution. 
The next example introduces the Duffing's equation and illustrates the case where a resonant term arises at $O\left(\epsilon^{2}\right)$.

Example: (Duffing's equation)

Let us consider an undamped nonlinear oscillator described by Duffing's equation

$$
u_{t t}+u+\epsilon u^{3}=0
$$

with $u(0)=1$ and $u_{t}(0)=0$.

In order to obtain a solution to the equation (2.47), we use the regular perturbation theory, where solution written as series in power of $\epsilon$ :

$$
u=u_{0}+\epsilon u_{1}+\epsilon^{2} u_{2}+\epsilon^{3} u_{3}+\cdots
$$

Substituting this expansion into equation (2.47), we get

$$
\left(u_{0 t t}+\epsilon u_{1 t t}+\epsilon^{2} u_{2 t t}+\cdots\right)+\left(u_{0}+\epsilon u_{1}+\epsilon^{2} u_{2}+\cdots\right)+\epsilon\left(u_{0}+\epsilon u_{1}+\epsilon^{2} u_{2}+\epsilon^{3} u_{3}+\cdots\right)^{3}=0
$$

Equating coefficients of the same power of $\epsilon$ leads to the following problem for $u_{0}$

$$
u_{0 t t}+u_{0}=0, u_{0}(0)=1 \text { and } u_{0 t}(0)=0
$$

Then, the solution for $u_{0}$ is

$$
u_{0}(t)=\cos (t)
$$

The problem for $u_{1}$ is given by

$$
u_{1 t t}+u_{1}+u_{0}^{3}=0, u_{1}(0)=0 \text { and } u_{1 t}(0)=0 .
$$


Substituting $u_{0}(t)$ from equation (2.51) and using the trigonometric identity for $\cos ^{3}(t)$ yields

$$
u_{1 t t}+u_{1}=-\frac{1}{4}(3 \cos (t)+\cos (3 t)) .
$$

This is an inhomogeneous second order differential equation and can be solved by using the variation of parameters method to obtain:

$$
u_{1}(t)=-\frac{3}{8} t \sin (t)-\frac{1}{32} \cos (t)+\frac{1}{32} \cos (3 t)
$$

Notice that the solution at order $O(\epsilon)$ contains a term that grows linearly in t; i.e., the solution is unbounded. This term is called a secular term. Therefore the expansion is not uniformly valid in $t$ and breaks down when $t=O\left(\epsilon^{-1}\right)$, since then $u_{0} \approx \epsilon u_{1}$.

To eliminate the secular term by using multiple scale analysis (see, for example Nayfeh [47]), we introduce a new time scale $\tau=\epsilon t$ and find solutions as a function of both $t$ and $\tau$ by considering them as independent variables.

Then, suppose the solution to be of the form

$$
u(t)=U_{0}(t, \tau)+\epsilon U_{1}(t, \tau)+\epsilon^{2} U_{2}(t, \tau)+O\left(\epsilon^{3}\right)
$$

Use the chain rule to obtain the derivatives of $u(t, \tau)$; e.g., the second derivative is

$$
\frac{d^{2} U_{i}}{d t^{2}}=\frac{\partial^{2} U_{i}}{\partial t^{2}}+2 \epsilon \frac{\partial U_{i}}{\partial \tau \partial t}+\epsilon^{2} \frac{\partial^{2} U_{i}}{\partial^{2} \tau}
$$

Substituting these derivatives into equation (2.47) and group terms of $O(1)$ and $O(\epsilon)$, we obtain

$$
\frac{\partial^{2} U_{0}}{\partial t^{2}}+U_{0}=0
$$


and

$$
\frac{\partial^{2} U_{1}}{\partial t^{2}}+U_{1}=-U_{0}^{3}-2 \frac{\partial^{2} U_{0}}{\partial t \partial \tau}
$$

The general solution of $(2.57)$ is

$$
U_{0}(t, \tau)=A(\tau) e^{i t}+A^{*}(\tau) e^{-i t}
$$

where $A(\tau)$ is an arbitrary complex function of $\tau$.

We substitute $U_{0}(t, \tau)$ into equation (2.58), but as $e^{i t}$ and $e^{-i t}$ are solutions for the corresponding homogeneous equation, in order to avoid the appearance of secularity, then $A(\tau)$ must satisfy

$$
-3 A^{2} A^{*}-2 i \frac{d A}{d \tau}=0, \quad-3 A\left(A^{*}\right)^{2}+2 i \frac{d A^{*}}{d \tau}=0
$$

In order to solve the first equation for $A(\tau)$, we consider $A(\tau)=R(\tau) e^{i \theta(\tau)}$, where $R$ and $\theta$ are real. By substituting this form into the first equation in (2.60) and equating the real and imaginary parts, we obtain

$$
\frac{d R}{d \tau}=0, \quad \frac{d \theta}{d \tau}=\frac{3}{2} R^{2}
$$

Therefore the solution of $A(\tau)$ is

$$
A(\tau)=R(0) e^{i\left[\theta(0)+\frac{3}{2} R^{2}(0) \tau\right]}
$$

Now, the leading-order $U_{0}$ in equation (2.59) becomes

$$
U_{0}(t, \tau)=2 R(0) \cos \left[\theta(0)+\frac{3}{2} R^{2}(0) \tau+t\right]
$$


By using the initial conditions, we can determine $R(0)$ and $\theta(0)$. Since $\tau=\epsilon t$, the solution of (2.47) is given by

$$
u(t)=\cos \left[t\left(1+\frac{3}{8} \epsilon\right)\right]+O(\epsilon)
$$

Wave motion in a medium is the movement of disturbances relative to the medium. The response of oscillations in acoustic systems near resonance can be continuous or shocked. The next section starts with a study of the continuous regime and discusses how some features connect to the nature of the response.

\subsection{Continuous Wave Motion}

In this section, we will discuss how resonant forcing of a gas contained between two concentric spheres can lead to a continuous motion. We are now going to present examples to illustrate the motion of a gas inside certain symmetric geometries, such as between concentric spheres.

\subsubsection{Resonant oscillations of an inhomogeneous gas between concentric spheres}

As explained in Chapter 1, the study of resonant oscillations of a gas in different bounded containers was generally discussed experimentally and theoretically. For more details on the different container of a gas, see for example $[9,10,12,14,16,25$, $53,59]$. In this section, we consider the example of nonlinear resonant oscillations of an inhomogeneous gas contained in a spherical shell, which is chosen for the sake of container.

Consider a homogeneous gas contained between two concentric spheres, with radii 
$0<r_{2} \leq r \leq r_{1}$, where $r_{1}$ is the external boundary and $r_{2}$ is the interior boundary that oscillates periodically at or near the fundamental frequency of the linear free vibration. The motion of the gas is assumed to be radially symmetric. However, the conversation of energy is not included here as the gas is isentropic. The undamped

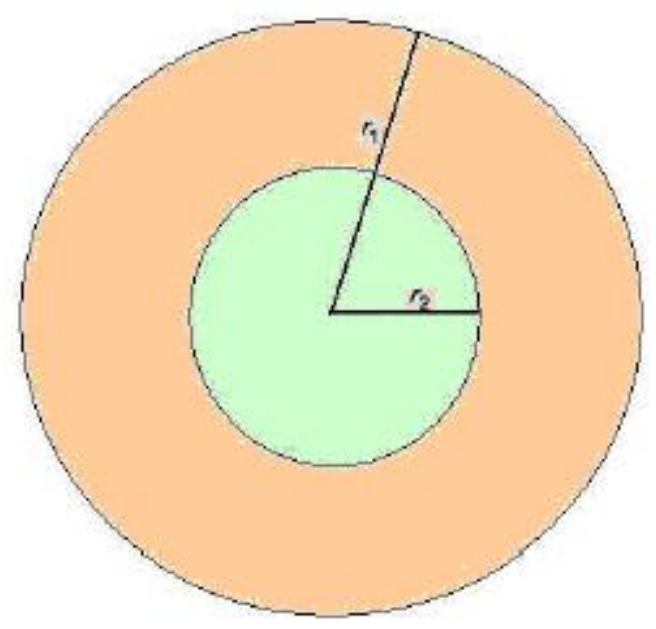

Figure 2.2: Spherical geometry.

governing equations in dimensional variables are given by

$$
\rho\left[\frac{\partial u}{\partial t}+u \frac{\partial u}{\partial r}\right]+\frac{\partial P}{\partial r}=0, \quad \frac{\partial \rho}{\partial t}+\frac{\partial u \rho}{\partial r}+\frac{2 u \rho}{r}=0
$$

The equation of state for the isentropic flow of an ideal gas is

$\frac{P}{P_{s}}=(1+e)^{\gamma}=1+\gamma e+\frac{\gamma(\gamma-1)}{2} e^{2}+\ldots$

Pressure and density are measured from their values in a reference state $\left(P_{s}, \rho_{s}\right)$, where the condensation $e(r, t)=\rho-1, \gamma$ is a specific heat ratio and $P_{s}$ is a constant. The velocity $u$ is nondimensionalized with respect to the sound speed $a_{s}(r)=\sqrt{\frac{\gamma P_{s}}{\rho_{s}}}$. The boundary conditions are

$$
u\left(r_{2}, t\right)=0, u\left(r_{1}, t\right)=\omega l \sin (\omega t)
$$

where $l$ is the maximum boundary displacement with frequency $\omega$. 
Assume the shell thickness, $r_{1}-r_{2}$, is fixed and $0 \leq x \leq 1$ is defined by $x=\frac{r}{r_{1}-r_{2}}-L$, where $L=\frac{r_{2}}{r_{1}-r_{2}}$. The dimensionless velocity, pressure and density are considered as functions of dimensionless length $x$ and time $\frac{t a_{0}}{r_{1}-r_{2}}$ respectively.

Now, the dimensionless form for the governing equations (2.65) becomes

$$
\begin{gathered}
\omega u_{\theta}+u u_{x}+(1+e)^{\gamma-2} e_{x}=0, \\
\omega e_{\theta}+[(1+e) u]_{x}+\frac{2(1+e) u}{L+x}=0,
\end{gathered}
$$

and equation (2.66) now is $u(0, \theta)=0, u(1, \theta)=M \sin \theta$, where $\theta=\omega t$ and the Mach number of the applied input velocity is $M=\frac{\omega l}{a_{0}} \ll 1$. It should be noted that the equations for a straight tube can be recovered by taking the limit as $L \rightarrow \infty$.

For convenience, a new dependent variable $w(x, \theta)$ is defined by

$$
w(x, \theta)=s(x) u(x, \theta)
$$

with

$$
s(x)=(L+x)^{2} .
$$

This yields the canonical form of the governing equations (2.67) and (2.68), which provide the basis for the perturbation scheme for continuous motion

$$
\begin{gathered}
\omega w_{\theta}+w\left(\frac{w}{s}\right)_{x}+s(1+e)^{\gamma-2} e_{x}=0 \\
\omega e_{\theta}+\left(\frac{1}{s}\right)[(1+e) w]_{x}=0 \\
w(0, \theta)=0, w(1, \theta)=\epsilon^{3} \sin \theta
\end{gathered}
$$

where $\epsilon^{3}=M(L+1)^{2}<<1$. We can approximately solve this nonlinear problem for a continuous output by using a perturbation expansion of the condensation, frequency, 
and new dependent variable as follows:

$$
\begin{gathered}
w(x, \theta)=\epsilon w_{1}(x, \theta)+\epsilon^{2} w_{2}(x, \theta)+\cdots, \\
e(x, \theta)=\epsilon e_{1}(x, \theta)+\epsilon^{2} e_{2}(x, \theta)+\cdots, \\
\omega(\epsilon)=\lambda+\epsilon^{2} \delta+\cdots,
\end{gathered}
$$

where $\left|e_{i}\right|,\left|w_{i}\right|=O(1)$, for $i \geq 1, \lambda$ is the fundamental frequency and $\epsilon^{2} \delta$ is a detuning.

Notice that, for the linear case, the output is $O(\epsilon)$ while the input is $O\left(\epsilon^{3}\right)$, and we suppose that the motion is dominated by the first harmonic. Hence, the linear problem at $O(\epsilon)$ can be obtained as follows:

$$
\begin{gathered}
\lambda \frac{\partial \omega_{1}}{\partial \theta}+s \frac{\partial e_{1}}{\partial x}=0, \\
\lambda \frac{\partial e_{1}}{\partial \theta}+\frac{1}{s} \frac{\partial \omega_{1}}{\partial x}=0, \\
w_{1}(0, \theta)=0, w_{1}(1, \theta)=0 .
\end{gathered}
$$

Eliminating $e_{1}$ from equation (2.77) yields the Webster horn equation for $w_{1}(x, \theta)$ :

$$
\lambda^{2} \frac{\partial^{2} w_{1}}{\partial \theta^{2}}-s \frac{\partial}{\partial x}\left(\frac{1}{s} \frac{\partial w_{1}}{\partial x}\right)=0
$$

To solve it exactly, let

$$
w_{1}(x, \theta)=A \varphi(x) \sin \theta \text { and } e_{1}(x, \theta)=\frac{A}{\lambda s(x)} \varphi^{\prime}(x) \cos \theta,
$$

where $A$ is at this point an arbitrary constant amplitude $\varphi(x)$ satisfies the eigenvalue problem:

$$
\frac{\lambda^{2}}{s(x)} \varphi+\frac{d}{d x}\left(\frac{1}{s(x)} \frac{d \varphi}{d x}\right)=0
$$


with $\varphi(0)=\varphi(1)=0$.

To simplify the calculation, we choose a particular case of $s(x)$

$$
s(x)=L^{2}(1+k x)^{2},
$$

where $L$ is spherical geometry parameter and $k$ is the density variable parameter.

Mortell and Seymour [44] give the exact solution for (2.81) with $s(x)$ given in (2.82), and it has the form

$$
\varphi(x)=(1+k x) F^{\prime}(x)-k F(x),
$$

where $F(x)$ satisfies:

$$
F^{\prime \prime}+\lambda^{2} F=0 .
$$

Then, by the boundary conditions $\varphi(0)=\varphi(1)=0$, the eigenvalue equation is given by

$$
\tan (\lambda)=\frac{\lambda}{1+\left(\frac{\lambda}{k}\right)^{2}(1+k)} .
$$

In general, the eigenvalues are incommensurate; i.e. the higher modes are not integer multiples of the fundamental $\left(\lambda_{n} \neq j \lambda_{1}, j \geq 2\right)$. Notice that, however, when $k=0$, the eigenvalues are commensurate. Strictly speaking, for $k \neq 0$, we have incommensurate eigenvalues.

Hence, the solution of the linear problem (2.77)-(2.78) is given by (2.80) where $\varphi(x)$ is given by equations (2.83)-(2.85). This implies that, to reach the complete solution, it is still necessary to determine the amplitude $A$. As will be seen, this requires consideration of the terms up to $O\left(\epsilon^{3}\right)$.

The second-order term in the perturbation expansion of equations (2.67) and (2.68) 
is given by:

$$
\begin{aligned}
\frac{\partial \omega_{2}}{\partial \theta}+s \frac{\partial e_{2}}{\partial x} & =-\omega_{1} \frac{\partial}{\partial x}\left(\frac{\omega_{1}}{s}\right)-(\gamma-2) s e_{1} \frac{\partial e_{1}}{\partial x} \\
\lambda \frac{\partial e_{2}}{\partial \theta}+\frac{1}{s} \frac{\partial \omega_{2}}{\partial x} & =-\frac{1}{s} \frac{\partial\left(e_{1} \omega_{1}\right)}{\partial x} \\
w_{2}(0, \theta) & =0, \quad w_{2}(1, \theta)=0 .
\end{aligned}
$$

By using the linear expansion in $(2.82)$, we can eliminate $e_{2}(x, \theta)$, and this implies that the second term should be in the form $w_{2}(x, \theta)=A^{2} B(x) \sin (2 \theta)$, where $B(x)$ satisfies:

$$
\frac{(2 \lambda)^{2}}{s} B+\frac{d}{d x}\left(\frac{1}{s} \frac{d B}{d x}\right)=\frac{\lambda \varphi}{s^{2}}\left[(\gamma+1) \varphi^{\prime}-\left(2 \frac{s^{\prime}}{s}+\frac{c^{\prime}}{s}\right) \varphi\right]
$$

with $B(0)=0, B(1)=0$, and $\varphi$ is the eigenfunction given by equations (2.83) and (2.84).

A natural question arises as to whether there are any restrictions on the existence of the function $B(x)$. The answer is $B(x)$ exists with no restriction on the amplitude $A$, provided $2 \lambda$ is not an eigenvalue of the operator on the left-hand side of (2.89). See the boundary theorem in Waltman [65].

Now the solution up to $O\left(\epsilon^{2}\right)$ is

$$
w(x, \theta)=\epsilon A \varphi(x) \sin \theta+\epsilon^{2} A^{2} B(x) \sin 2 \theta .
$$

Thus, the velocity $u(x, \theta)$ can be found uniquely by using equation (2.69).

The equation at $O\left(\epsilon^{3}\right)$ to determine $w_{3}(x, \theta)$ is given by

$$
\lambda^{2} \frac{\partial^{2} w_{3}}{\partial \theta^{2}}-s \frac{\partial}{\partial x}\left(\frac{1}{s} \frac{\partial w_{3}}{\partial x}\right)=\left[-2 \lambda \delta A \varphi+A^{3} C_{1}(x)\right] \sin \theta+A^{3} C_{2}(x) \sin 3 \theta
$$

with $w_{3}(0, \theta)=0$ and $w_{3}(1, \theta)=\sin \theta$, where $C_{1}(x)$ and $C_{2}(x)$ depend on $s(x), w_{1}$, and $w_{2}$. 
Assume the solution is in the form $w_{3}(x, \theta)=P(x) \sin \theta+Q(x) \sin 3 \theta$. After substituting this in equation (2.91) and equating the coefficients of trigonometric functions on both sides of the equation, we get the following non-homogeneous ordinary differential equation for $Q(x)$ :

$$
(3 \lambda)^{2} Q+s \frac{d}{d x}\left(\frac{1}{s} \frac{d Q}{d x}\right)=-A^{3} C_{2}(x) \sin 3 \theta
$$

with $Q(0)=0$, and $Q(1)=0$.

Provided $3 \lambda$ is not an eigenvalue of the operator in $(2.92)$, then the solution for $Q(x)$ exists with no restriction on $A$. However, the resulting equation for $P(x)$ is given by:

$$
\frac{\lambda^{2}}{s} P+\frac{d}{d x}\left(\frac{1}{s} \frac{d P}{d x}\right)=-2 \lambda \delta A \varphi s^{-1}+A^{3} C_{4}(x)
$$

where $C_{4}(x)=\frac{C_{1}(x)}{-s}$, with $P(0)=0$ and $P(1)=1$.

The solution for $P(x)$ exists only if the amplitude $A$ satisfies the following solvability condition as a consequence of the Fredholm alternative:

$$
N_{1} A^{3}-2 \lambda \delta A=N_{2}
$$

where

$$
N_{1}=\int_{0}^{1}\left[Q_{1}(x)+Q_{2}(x) B(x)\right] d x, \text { and } N_{2}=\int_{0}^{1} \frac{\varphi}{s}\left[\lambda^{2} x-\frac{s^{\prime}}{s}-\frac{c^{\prime}}{c}\right] d x
$$

where $Q_{1}(x)$ and $Q_{2}(x)$ are functions of $s^{\prime}, s, c^{\prime}, c, \varphi$ and $\varphi^{\prime}$.

The solution for $w_{1}(x, \theta)$ is complete and valid provided $|\epsilon A B|<<1$. Hence, the motion is dominated by the first harmonic. However, as we have seen, it is crucial that the linear eigenvalues be incommensurate. 
Before we consider the case where the eigenvalues are commensurate, we note some different limits of the eigenvalue equation to explain how they correspond to some previous results for simpler geometries such as a full sphere, a cone and a straight tube.

\section{Asymptotic forms of the eigenvalue equation}

The resulting eigenvalue equation in (2.85) that determines the various resonant modes is crucial for understanding the shock formation. Chester in 1991 [8] studied the case of a homogeneous gas for full sphere and the plane-wave case for a shell of internal radius $L$ and fixed thickness as we will discuss in this subsection.

\section{Homogeneous gas}

In the case where $k L=1$, the gas is homogeneous. This implies the eigenvalue equation (2.85) contains only the effect of the spherical geometry and becomes:

$$
\tan (\lambda)=\frac{\lambda}{1+(\lambda L)^{2}\left(1+L^{-1}\right)}
$$

This eigenvalue equation explains how the effect of the geometry plays a major role in the nature of the spectrum regarding the commensurate property. Now, we will discuss the following two examples to clarify more this relation.

\section{Full sphere}

Consider the case where the limit $L \rightarrow 0$. Then this corresponds to a full sphere containing a homogeneous gas. Since the thickness is fixed, the eigenvalue equation (2.96) becomes $\tan (\lambda)=\lambda$. This result coincides with the equation given by Ellermeier (see [19]) and yields incommensurate eigenvalues. 
Plane wave

As an example that helps us to understand the effect of geometry, we also consider the limit $L \rightarrow \infty$. When fixed shell thickness becomes small compared with the internal radius of the shell, and the radial lines become parallel within the shell, then the eigenvalue equation (2.96) reduces to $\tan (\lambda)=0$ with the solution $\lambda=\lambda_{n}=n \pi$, where $n \geq 1$. This means the eigenvalues are commensurate. It is also well known that in this case shocks form.

Next, it will be explained why in the shocked regime there is a need to use a different approach called the nonlinear geometrical acoustic approximation to obtain the analytic solution.

\subsection{Shocked Wave Motion}

Continuous nonlinear resonant oscillations in shaped resonators are described by using a dominant first-harmonic approximation as seen in the previous section. In the following part, the focus is on nonlinear resonant oscillation of an inhomogeneous gas contained between two concentric spheres when the dominant first-harmonic approximation is not valid and the flow contains shocks. A number of studies have been discussed the existence of shock, see [27, 42]. One of the most important questions is what happens if the dominant first harmonic approximation is not valid. Recently, Seymour et al. [57] have shown that a nonlinear geometric acoustics approximation predicts shocked flows between concentric spheres and coaxial cylinders. They compared the results with full numerical solutions and found good agreement.

In the case of concentric spheres or coaxial cylinders, the effect of geometry may be investigated through a dimensionless parameter $L$, which is the ratio of the inner radius to the distance between the spheres or cylinders. Chester [8] showed that, when $L \rightarrow 0$ and the Mach number is small, the flow is continuous. But whenever the 
nonlinearity is dominant due to the increasing in the Mach number, shocks appear in the flow. However, if $L \gg 1$ and the flow is radially symmetric, then the motion of the gas between the radial lines can be considered as approximating the flow in a closed tube. Hence, for large $L$, the gas flow is similar to that in a closed-ended straight tube, and it will contain shocks.

We explain now how using the nonlinear geometrical acoustic approximation is useful to describe the analytic solution for a gas contained between two concentric spheres when a shock is present. For more details see, for instance, [63, 60].

\subsubsection{Sphere}

Consider a gas contained between two concentric spheres, similar to the continuous case in Section 2.3 where the constant reference pressure and density are $P_{s}$ and $\rho_{s}$, respectively. We are focused on the limit as $L \rightarrow \infty$. The governing equations in Eulerian coordinates are given by (2.67) and (2.68) with the boundary conditions $u(0, \theta)=0, u(1, \theta)=M^{2} \sin \theta$, where $\theta=\omega t$ but now the Mach number of the applied input velocity is $M^{2}=\frac{\omega l}{a_{0}} \ll 1$.

After using the same procedure in the previous section and substituting a new dependent variable $w(x, \theta)$ from equation (2.69) into the governing equations (2.67) and (2.68), the same canonical form equations (2.71) and (2.72) are obtained. The boundary conditions become

$$
w(0, \theta)=0, w(1, \theta)=A^{2} \sin \theta
$$

where $A^{2}=M^{2}(L+1)^{2} \ll 1$. Notice that the difference between this case and the continuous case in Section 2.3 is that these ordering parameters are slightly different. In fact, for the shocked case, we choose the scale $A$; i.e., in anticipation of the response being $O(A)$. 


\section{Nonlinear Geometrical Acoustic Approximation (Sphere)}

The effect of the geometry in the governing equations (2.67) and (2.68) is contained in the term $s(x)=(L+x)^{2}$. It is convenient to change the variables from $(x, \theta)$ to $(x, \alpha)$ to get a more simplified system when studying the propagation of waves between the spheres, where $\alpha(x, T)=$ constant is the nonlinear characteristic variable associated with outward waves and $T(x, \alpha)$ is the arrival time of the outward wave at location $x$. Let $T(x, \alpha)=x+\alpha$, where $\alpha=T$ on $x=0$, and if we suppose that $w(x, \theta)=W(x, \alpha)$, then $w_{x}=W_{x}-\alpha_{T} T_{x} W_{\alpha}$.

Then, equations (2.71) and (2.72) become

$$
\begin{aligned}
& \left(1-T_{x} \frac{W}{s}\right) W_{\alpha}-s T_{x}(1+E)^{\gamma-2} E_{\alpha}=-\frac{s}{\alpha_{T}} E_{x}+\text { nonlinearterms } \\
& \left(1-T_{x} \frac{W}{s}\right) E_{\alpha}-\frac{T_{x}}{s}(1+E) W_{\alpha}=-\frac{1}{s \alpha_{T}} W_{x}+\text { nonlinearterms }
\end{aligned}
$$

This yields the characteristic condition

$$
T_{x}= \pm\left[(1+E)^{\frac{\gamma-1}{2}} \mp \frac{W}{s}\right]^{-1}= \pm\left[1-\frac{\gamma-1}{2} E \mp \frac{W}{s}+O\left(E^{2}\right)\right]
$$

Consider the outgoing wave for the nonlinear geometrical acoustics expansion [57]:

$$
\begin{aligned}
W & =\sum_{n=0}^{n} \chi^{n} W_{n}(x) f_{n}(\alpha), E=\sum_{n=0}^{n} \chi^{n} E_{n}(x) f_{n}(\alpha), \\
T & =\alpha+x+\chi \sum_{n=0} \chi^{n} T_{n}(x, \alpha)
\end{aligned}
$$

where $\chi$ is a small amplitude parameter. Then, to order $\chi$ for a wave travelling 
outwards:

$$
W_{1}(x)=s(x) E_{1}(x), f_{2}^{\prime}(\alpha)=f_{1}(\alpha), W_{1}^{\prime}(x)=-s(x) E_{1}^{\prime}(x)
$$

Hence

$$
W(x)=\sqrt{s(x)} f(\alpha), E=\frac{f(\alpha)}{\sqrt{s(x)}},
$$

where $f_{1}(\alpha)=f(\alpha)$. Then, from equation (2.100) and using $s(x)$ in equation (2.70), the corresponding nonlinear right-travelling characteristic is:

$$
T=\alpha+x-\chi \frac{\gamma+1}{2} f(\alpha) \ln \left(\frac{L+x}{L}\right)
$$

After we linearize equations (2.71) and (2.72) and eliminate $w$, the equation for $e(x, t)$ is given by the following spherical wave equation

$$
\frac{\partial^{2}}{\partial t^{2}}[\sqrt{s} e]-\frac{\partial^{2}}{\partial x^{2}}[\sqrt{s} e]=0
$$

Therefore, for an outgoing wave, the exact solution of equation (2.105) with $s(x)$ in equation $(2.70)$ is

$$
e(x, t)=\frac{1}{\sqrt{s(x)}} \frac{\partial F}{\partial x}, \text { with } w=\sqrt{s(x)} \frac{\partial F}{\partial x}+F
$$

where $F(t, x)$ is an arbitrary function of the linear characteristic $t-x$. Thus, the condensation $e(x, t)$ propagates as a nonlinear spherical wave with the radius $\sqrt{s(x)}$. Now for a wave travelling inwards, let $T(x, \beta)$ be the arrival time of inward wave at location $x$ corresponding to constant $\beta$, where the condition is $\beta=T$ at $x=1$. Using a similar procedure to the outward wave, we have:

$$
W(x)=\sqrt{s(x)} g(\beta), E=-\frac{g(\beta)}{\sqrt{s(x)}} .
$$


Then the characteristic condition (2.100) becomes:

$$
T_{x}=-1-\chi \frac{\gamma+1}{2} \frac{1}{\sqrt{s}} g(\beta)
$$

and

$$
T=\beta-(x-1)-\chi \frac{\gamma+1}{2} g(\beta) \ln \left(\frac{L+x}{L+1}\right) .
$$

Since the motion of the gas between the spheres can be approximated by two modulated simple waves, see [61]. We obtain, from equations (2.103) and (2.107),

$$
W(\alpha, \beta, x)=\sqrt{s(x)}[f(\alpha)+g(\beta)], E(\alpha, \beta, x)=\frac{1}{\sqrt{s(x)}}[f(\alpha)-g(\beta)],
$$

where the two functions $f$ and $g$ can be determined from the boundary conditions at $x=0$ and $x=1$ :

$$
W(0)=0, W(1)=\chi \sin \theta
$$

Notice that, the amplitude of the forcing function is $\chi=A^{2}$; i.e., $A^{2}=\frac{\omega l}{a_{0}}(L+1)^{2}$. Then $g(\eta)$ satisfies the difference equation :

$$
g(\eta)-g(s)=\frac{A^{2}}{L+1} \sin (\eta), \eta=s+2 \omega+g(\eta)(\gamma+1) \omega \ln \left(\frac{L+1}{L}\right)
$$

where $2 \omega=2 \pi+\Delta, \Delta$ is the detuning and $\omega=\pi$ is the fundamental frequency.

Defining

$$
G(\eta)=g(\eta)(\gamma+1) \omega \ln \left(\frac{L+1}{L}\right)+\Delta
$$

$G(\eta)$ satisfies the conditions:

$$
G(\eta)-G(s)=B \sin (\eta), \eta=s+G(s)
$$


with the similarity parameter:

$$
B=\frac{(\gamma+1) A^{2} \omega}{L+1} \ln \left(\frac{L+1}{L}\right)
$$

The form of the function $G$ in (2.113) satisfies the conditions

$$
G(\eta)=G(\eta+2 \pi), \int_{0}^{2 \pi} G(\eta) d \eta=2 \pi \Delta .
$$

Equation (2.114) can be approximated to obtain:

$$
G(s)= \begin{cases}2 \sqrt{B} \sin \left(\frac{s}{2}\right) & \text { if } 0 \leq s<s_{1} \\ -2 \sqrt{B} \sin \left(\frac{s}{2}\right) & \text { if } s_{1}<s \leq 2 \pi\end{cases}
$$

where $s_{1}$ is the shock position which is determined by the mean condition in (2.116) in terms of the detuning and $B$ is given by equation (2.115). It is clear from $G(s)$ that the shock strength in $G$ is $4 \sqrt{B} \sin \left(\frac{s_{1}}{2}\right)$, and it should be noted that $B$ is of order $O(A)$, where $A$ is the amplitude of forcing given in equation (2.97). Figure 2.3 from Amundsen et al. [57] shows the comparison between the numerical solution for velocity $u(\theta)$ at $x=0.5$ with the geometrical acoustics approximation when $L=30$ and the output Mach number is $M=0.005$. 


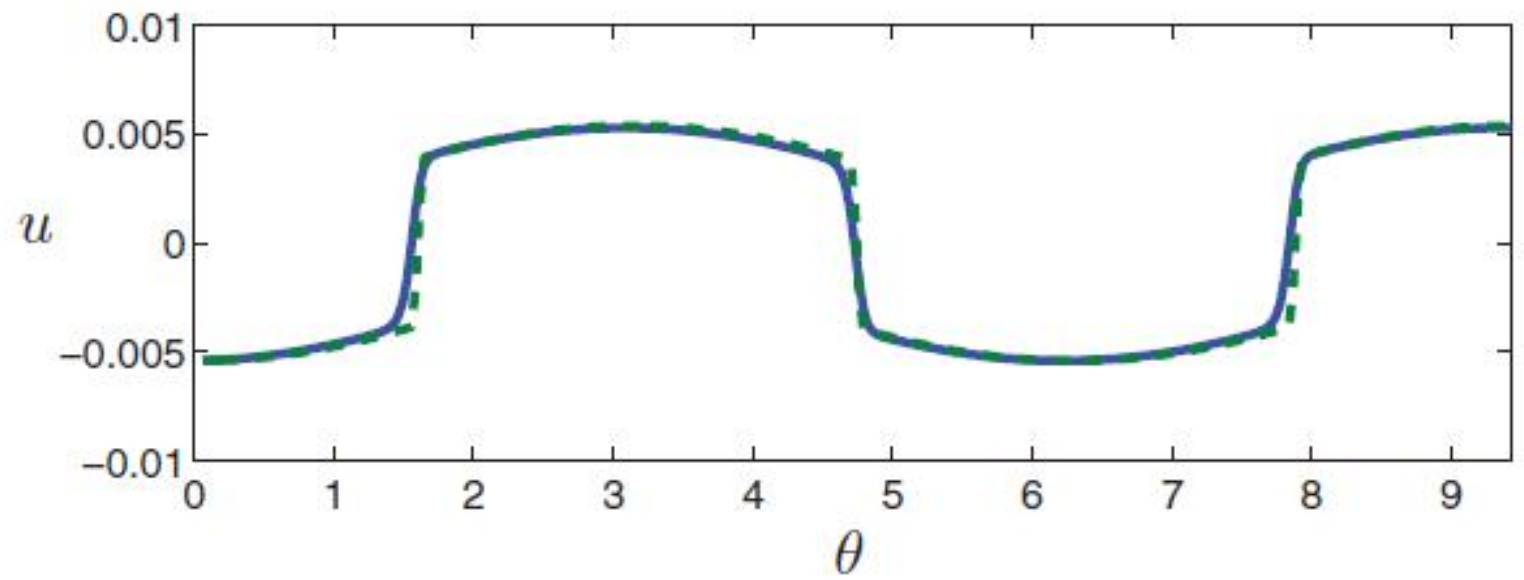

Figure 2.3: Figure 1 from Amundsen [57] illustrating the comparison of numerical solution (solid) and geometric acoustic approximation (dashed) for gas in spherical shell $L=30, M=0.005$ for $u(0.5, \theta)$. 


\section{Chapter 3}

\section{The Transition between Continuous and Unbounded Regimes}

In the previous chapter, we discussed two different regimes for resonant response in acoustic-wave systems, the first being the continuous response, and the second corresponding to an unbounded response. We additionally explained the correlation between the continuous solutions and incommensurate eigenvalues and between unbounded and commensurate eigenvalues. However, this correlation is not strict, and the transition between these two regimes is not well understood. In particular, the specific form of the nonlinearity can serve to overcome some degree of incommensurate nature in the eigenvalues, or not.

Before studying the transition between these two regimes, let us briefly define what the meaning of a shock is. Physically it corresponds to a sudden and violent change in the state of motion in parts of the medium. However, mathematically a shock 
solution is the term used to describe the type of weak solution where the assumption of regularity of the solution breaks down. So, typically, a shock is represented by a discontinuity where locally the solution undergoes a change in value.

Through this chapter, we will present a variety of model problems that illustrate this transition, and which will form a basis for continued study.

\subsection{Formulation of Nonlinear model problems}

In this section, we will introduce model problems that help us to understand the transition between unbounded and continuous response. Our goal is to consider the nature of the eigenvalue spectrum (i.e., is it commensurate or incommensurate), as well as using a numerical approximation to study the relation between the eigenvalues and the response.

Consider the following model problem

$$
u_{t t}-u_{x x}+f(u)=0
$$

where $f(u)$ is a nonlinear function of $u$, over the domain $x \in[0,1]$ and $t \in 0, \infty)$

The boundary conditions are

$$
u(0, t)=m \sin (\lambda t), \quad(\alpha-1) u(1, t)-\alpha u_{x}(1, t)=0,
$$

where $m$ is the forcing amplitude with frequency $\lambda$, and $\alpha$ is an arbitrary parameter.

For the case $\alpha=1$, we have $u_{x}(1, \theta)=0$, which in the context of a gas in a tube corresponds to the end being open. However, when $\alpha=0$, we obtain $u(1, \theta)=0$, and this corresponds to the case where the boundary is closed. 
For convenience, we suppose that $\theta=\lambda t$. Then equations (3.1) and (3.2) become

$$
\begin{gathered}
\lambda^{2} u_{\theta \theta}-u_{x x}+f(u)=0 \\
u(0, \theta)=m \sin (\theta),(\alpha-1) u(1, \theta)-\alpha u_{x}(1, \theta)=0 .
\end{gathered}
$$

The dimensionless form of the governing equations of gas dynamics given by (2.67) -(2.68) can be written in the model problem in the equation (3.3) by considering $f(u)=u_{x} u_{x x}$.

We will study some cases of the nonlinear terms $f(u)$ and explain how this form plays a significant role in the outcome response relative to the linear eigenvalue spectrum.

\subsubsection{Nonlinear form $f(u)=u^{n} u_{x}$}

This nonlinear form provides a simple basis to study the impact of nonlinear effects and is one that arises naturally in various settings. With this nonlinear form, equation (3.3) is now

$$
\lambda^{2} u_{\theta \theta}-u_{x x}+u^{n} u_{x}=0 .
$$

In the following example, we start by considering a case when $n=1$.

Example 1: $n=1$

The nonlinear model problem in this case is given by

$$
\lambda^{2} u_{\theta \theta}-u_{x x}+u u_{x}=0
$$

where the boundary conditions still given by equation (3.4). 


\section{Linear Theory}

The equations (3.4) and (3.6) provide the basis of a perturbation scheme, where $m$ is the perturbation parameter. In order to solve this nonlinear problem, we introduce the following perturbation expansions:

$$
\begin{aligned}
u(x, \theta) & =m^{\frac{1}{3}} u_{1}(x, \theta)+m^{\frac{2}{3}} u_{2}(x, \theta)+m u_{3}(x, \theta)+\cdots, \\
\lambda & =\omega_{0}+m^{\frac{1}{3}} \omega_{1}+m^{\frac{2}{3}} \omega_{2}+m \omega_{3}+\cdots,
\end{aligned}
$$

where $\left|u_{i}\right|=O(1), i=1,2,3, \ldots, \omega_{0}$ is the fundamental frequency. Notice that the assumption here is that the output is $O\left(m^{\frac{1}{3}}\right)$ while the input is $O(m)$, which is similar to that used in Section 3.2. Therefore, at this point, we need to assume that $m \ll 1$ to ensure the validity of this analysis.

The linear problem at $O(\epsilon)$, where $\epsilon=m^{\frac{1}{3}}$, is given by:

$$
\omega_{0}^{2} u_{1 \theta \theta}-u_{1 x x}=0
$$

and

$$
u_{1}(0, \theta)=0,(\alpha-1) u_{1}(1, \theta)-\alpha u_{1 x}(1, \theta)=0
$$

Now let the solution be $u_{1}(x, \theta)=A \phi(x) \sin (\theta)$, where $A$ is the amplitude. Substituting the second derivatives of $u_{1}$ with respect to $x$ and $\theta$ in equation (3.9) implies $\phi(x)$ satisfies the eigenvalue problem

$$
\omega_{0}^{2} \phi+\phi^{\prime \prime}=0, \phi(0)=0 \text {, and }(\alpha-1) \phi(1)=\alpha \phi^{\prime}(1) .
$$


Thus the eigenfunction takes the form

$$
\phi(x)=B \cos \left(\omega_{0} x\right)+C \sin \left(\omega_{0} x\right)
$$

and, upon applying the boundary condition at (3.11), we get that $\omega_{0}$ is determined by the eigenvalue equation

$$
\tan \left(\omega_{0}\right)=\frac{\alpha}{(\alpha-1)} \omega_{0}
$$

In general, the eigenvalues of this equation are incommensurate. However, the nature of the spectrum depends on the arbitrary value of $\alpha$; e.g., when $\alpha=0$ and $\alpha=1$, we obtain commensurate eigenvalues, but for $0<\alpha<1$, we obtain incommensurate eigenvalues.

Therefore the solution is given by

$$
u_{1}(x, \theta)=A \sin \left(\omega_{0} x\right) \sin (\theta)
$$

where $A$ is the amplitude.

From this equation for the linear solution, it is clear that the only remaining aspect to determine is the amplitude A. For this, as will be seen, we need to consider the nonlinear terms up to $O\left(\epsilon^{3}\right)$.

\section{Calculation of Amplitude A}

The first correction $u_{2}$ can be obtained by considering the terms of $O\left(\epsilon^{2}\right)$ in the perturbation solution to equation (3.6). We find that

$$
\begin{aligned}
\omega_{0}^{2} u_{2 \theta \theta}-u_{2 x x} & =-2 \omega_{0} \omega_{1} u_{1 \theta \theta}-u_{1} u_{1 x} \\
u_{2}(0, \theta) & =0,(\alpha-1) \quad u_{2}(1, \theta)-\alpha u_{2 x}(1, \theta)=0 .
\end{aligned}
$$


The solution of $u_{2}(x, \theta)$ can be obtained by using an appropriate orthogonality condition. We seek periodic solutions that have the same period as the boundary forcing. After multiplying equation (3.15) by $\sin \left(\lambda_{0} x\right) \sin (\theta)$ and integrating both sides over $0 \leq x \leq 1,0 \leq \theta \leq 2 \pi$, we obtain

$$
\omega_{1} \frac{\pi}{2}\left(2 \omega_{0}-\sin \left(2 \omega_{0}\right)\right)=0
$$

Hence the value of $\omega_{1}$ may be taken to be zero. Then the perturbation expansion for $\lambda$ becomes

$$
\lambda=\omega_{0}+\epsilon^{2} \delta+\cdots,
$$

where $\delta=\omega_{2}$. The balance at $O\left(\epsilon^{2}\right)$ can be written now as

$$
\omega_{0}^{2} u_{2 \theta \theta}-u_{2 x x}=-u_{1} u_{1 x}
$$

Substituting $u_{1}(x, \theta)$ at (3.14) into equation (3.19), we obtain

$$
\omega_{0}^{2} u_{2 \theta \theta}-u_{2 x x}=-\frac{A^{2}}{2} \phi(x) \phi^{\prime}(x)+\frac{A^{2}}{2} \phi(x) \phi^{\prime}(x) \cos (2 \theta) .
$$

It is clear that the form of $u_{2}(x, \theta)$ must be $u_{2}(x, \theta)=C(x)+B(x) \cos (2 \theta)$. Substituting this form of $u_{2}(x, \theta)$ into equation (3.19), then equating the coefficients of $\cos (2 \theta)$, we find $B(x)$ is given by the following equation:

$$
B^{\prime \prime}(x)+\left(2 \omega_{0}\right)^{2} B(x)=-\frac{A^{2}}{2} \phi(x) \phi^{\prime}(x),
$$

with $B(0)=0, B(1)=\frac{\alpha}{\alpha-1} B^{\prime}(1)$. 
We should note here that $\phi(x)$ is the eigenfunction given by the equation (3.12). Now $2 \omega_{0}$ is not an eigenvalue, as we assumed the eigenvalues are incommensurate. After solving this equation for $B(x)$, we get

$$
\begin{aligned}
B(x)= & \frac{A^{2}}{32 \sin \left(2 \omega_{0}\right)(1-\alpha)+64 \cos \left(2 \omega_{0}\right) \alpha \omega_{0}}\left[(x \alpha-1-x) \sin \left(2 \omega_{0}(x-1)\right)\right. \\
& +2 \omega_{0} \alpha(x+1) \cos \left(2 \omega_{0}(x-1)\right)+(x-1-x \alpha) \sin \left(2 \omega_{0}(x+1)\right) \\
& \left.+2 \omega_{0} \alpha(1-x) \cos \left(2 \omega_{0}(x+1)\right)\right] .
\end{aligned}
$$

However, when equating the coefficients of non-trigonometric terms, we find that $C(x)$ is determined by

$$
C^{\prime \prime}(x)=\frac{A^{2}}{2} \phi(x) \phi^{\prime}(x)
$$

with $C(0)=0$ and $C(1)=\frac{\alpha}{\alpha-1} C^{\prime}(1)$, and where $(.)^{\prime}$ means the derivative with respect to $x$.

Using $\phi(x)=\sin \left(\omega_{0} x\right)$, we obtain the following solution for $C(x)$

$$
C(x)=\frac{A^{2}}{16 \omega_{0}}\left[x\left(2 \omega_{0} \alpha \cos \left(2 \omega_{0}\right)+(1-\alpha) \sin \left(2 \omega_{0}\right)\right)-\sin \left(2 \omega_{0} x\right)\right] .
$$

Now the solution of $u_{2}(x, \theta)=C(x)+B(x) \cos (2 \theta)$ is complete, where $C(x)$ and $B(x)$ are given by equations (3.22) and (3.24) respectively.

The balance at $O\left(\epsilon^{3}\right)$ is of the form

$$
\omega_{0}^{2} u_{3 \theta \theta}-u_{3 x x}+u_{1} u_{2 x}+u_{2} u_{1 x}+2 \omega_{0} \delta u_{1 \theta \theta}=0
$$

and

$$
u_{3}(0, \theta)=\sin (\theta), \quad(\alpha-1) u_{3}(1, \theta)-\alpha u_{3 x}(1, \theta)=0
$$

After multiplying equation (3.25) by $u_{1}(x, \theta)$ and integrating both sides for $0 \leq x \leq 1$, 
$0 \leq \theta \leq 2 \pi$, then using the boundary condition in equation (3.26), we obtain the solvability condition, which puts the following restriction on $A$ :

$$
N_{1}\left(\alpha, \omega_{0}\right) A^{3}+N_{2}\left(\alpha, \omega_{0}\right) A \delta-\omega_{0} \pi=0
$$

where $N_{1}$ and $N_{2}$ are given by

$$
\begin{aligned}
N_{1}\left(\alpha, \omega_{0}\right) & =\frac{1}{4096 \omega_{0}^{2} \alpha^{3}(\alpha-1)\left(1+\cos \left(2 \omega_{0}\right)\right)}\left(\alpha \pi \left(-24 \pi \alpha^{3} \omega_{0}^{2}-\left(5 \pi-24 \pi \omega_{0}^{2}\right) \alpha^{2}+5 \pi \alpha\right.\right. \\
& +80 \alpha+24) \cos \left(6 \omega_{0}\right)+8 \alpha \pi\left(-4 \omega_{0}^{2} \alpha^{3}-15 \alpha-8+\left(5+4 \omega_{0}^{2}\right) \alpha^{2}\right) \cos \left(4 \omega_{0}\right) \\
& +\pi\left(32-\left(152 \omega_{0}^{2}+11\right) \alpha^{3}+\left(64 \omega_{0}^{2}+259\right) \alpha^{2}+88 \omega_{0}^{2} \alpha^{4}\right) \cos \left(2 \omega_{0}\right)+8 \alpha \pi\left(12 \omega_{0}^{2} \alpha^{3}\right. \\
& \left.-\left(20 \omega_{0}^{2}+7\right) \alpha^{2}+\left(8 \omega_{0}^{2}-6\right) \alpha-17\right), \\
N_{2}\left(\alpha, \omega_{0}\right) & =\frac{1}{4096 \omega_{0}^{2} \alpha^{3}(\alpha-1)\left(1+\cos \left(2 \omega_{0}\right)\right)}\left(-1024 \pi \alpha^{2} \omega_{0}(\alpha-1) \cos \left(4 \omega_{0}\right)\right. \\
& \left.+\left(4096 \pi \alpha \omega_{0}(\alpha-1)\right) \cos \left(2 \omega_{0}\right)+1024 \alpha \omega_{0} \pi(\alpha-1)(\alpha-4)\right) .
\end{aligned}
$$

For example, when $\alpha=2$, the restriction of the amplitude $A$ is given by

$$
0.46858 A^{3}-14.12738532 A \delta-\omega_{0} \pi=0
$$

This equation will yield the amplitude-frequency relation, and it will be used to obtain the amplitude $A$ at the leading-order approximation for $u(x, \theta)$. In particular, we may choose $\delta=0$ in the cubic equation (3.29) to obtain $A=-3.13694$. It should be noted that the negative value of the amplitude is due to a phase shift.

Now we examine the following cases: 
Case 1: $\alpha=0$.

Consequently, the eigenvalue equation (3.13) becomes $\tan \left(\omega_{0}\right)=0$. Thus the eigenvalues are commensurate and are given by $\omega_{0}=\lambda_{n}=n \pi$, where $n \geq 1$. For the purpose of numerical approximation, we let $m=0.0001$. We apply the Runge-Kutta method of order four to discretize the time variable and the central difference formula to approximate the partial derivative with respect to $x$. Then the numerical simulation is carried out with the boundary conditions $u(0, \theta)=m \sin (\theta)$ and $u(1, \theta)=0$.

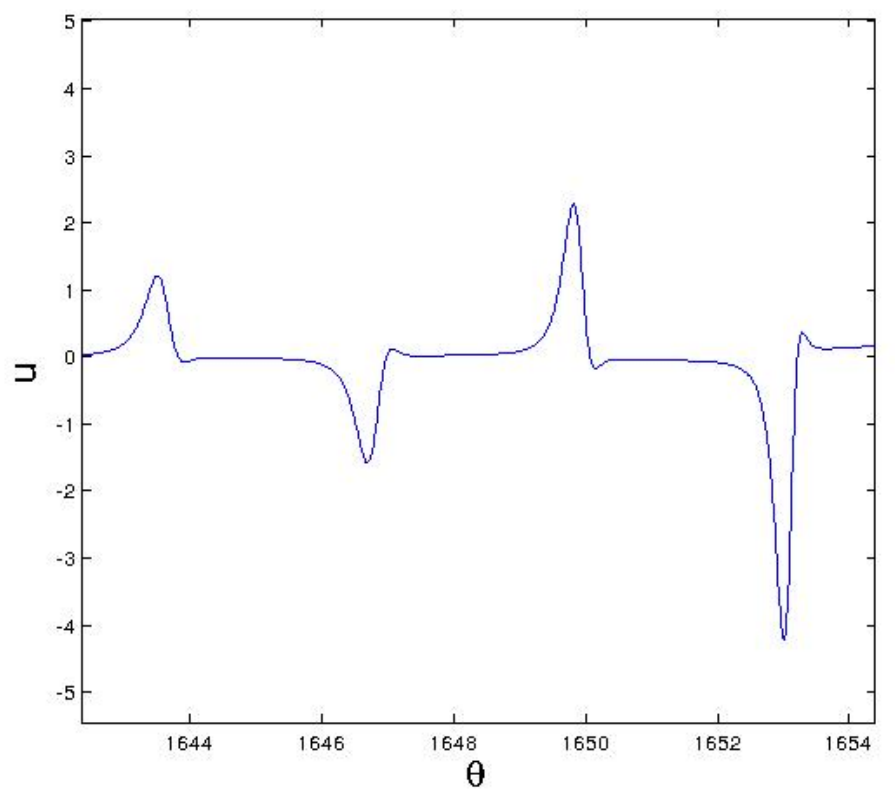

Figure 3.1: Plot of $u(x, \theta)$ as a function of $\theta$ where $\alpha=0$ at $x=0.5$.

Figure 3.1 shows the simulation result for this case where the response is unbounded. This case also explains the correlation between the commensurate eigenvalue and unbounded regime. 
Case 2: $\alpha=2$.

Notice that, in this case, the higher modes of the eigenvalue equation given by (3.13) are not integer multiples of the fundamental frequency. Hence, the eigenvalues are incommensurate.

Choosing $m=0.0001$ and $\alpha=2$, we see that the solution using a numerical method as in Case 1 achieves continuous response. We can also apply the perturbation technique as discussed above to find a leading-order approximate solution. Figure 3.2 shows a comparison and good agreement between these approximate solutions and the exact numerical solution.

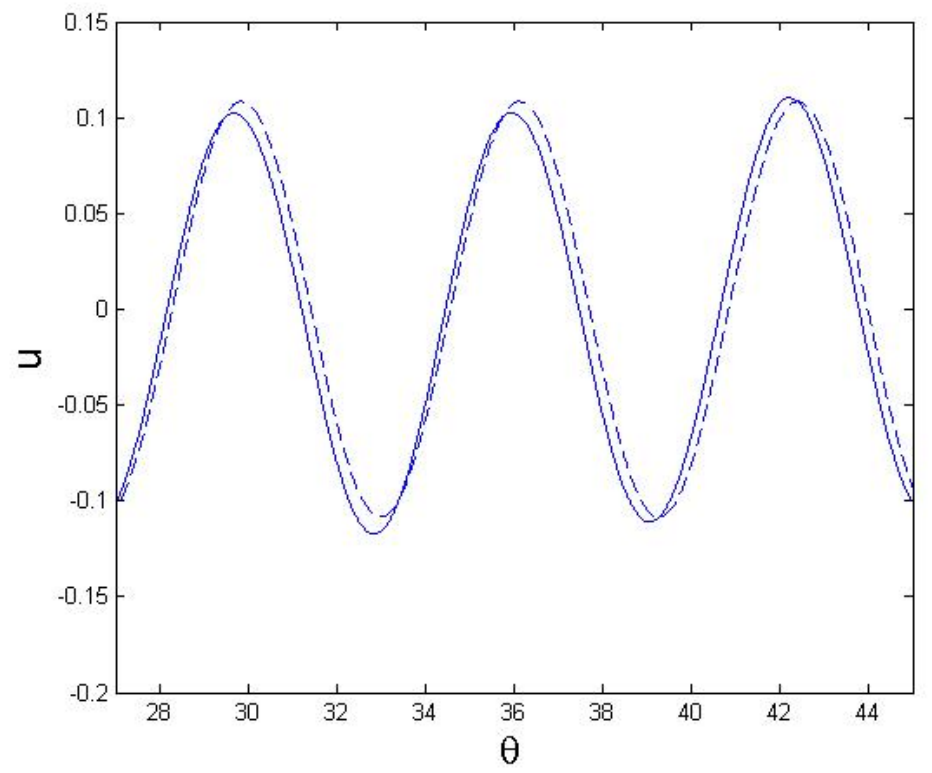

Figure 3.2: Comparison of numerical solution (solid line) to leading-order approximation (dashed line) $m=0.0001$ with $\alpha=2$, and the solution of (3.29). 
Case 3: $\alpha=1$.

In this case, the fundamental resonant frequency is $\omega_{0}=\frac{\pi}{2}$, with higher spectrum $\lambda_{n}=$ $\frac{(2 n+1) \pi}{2}=(2 n+1) \omega_{0}$, where $n \geq 1$. That implies the eigenvalues are commensurate. But we should note that these commensurate eigenvalues consist of only odd modes. We can vary the parameter $\alpha$ and go from one type of commensurate situation that has integer multiples to another type that has odd integer multiples. We again use a very similar method to the one utilized in the previous two cases to approximate the solution of equations (3.6). In this case, applying the boundary condition $u_{x}(1, \theta)=0$, we obtain a continuous response as shown in Figure 3.3. However, the analytical approximation solution for this case will be studied in detail in the next chapter and compared with the exact numerical solution.

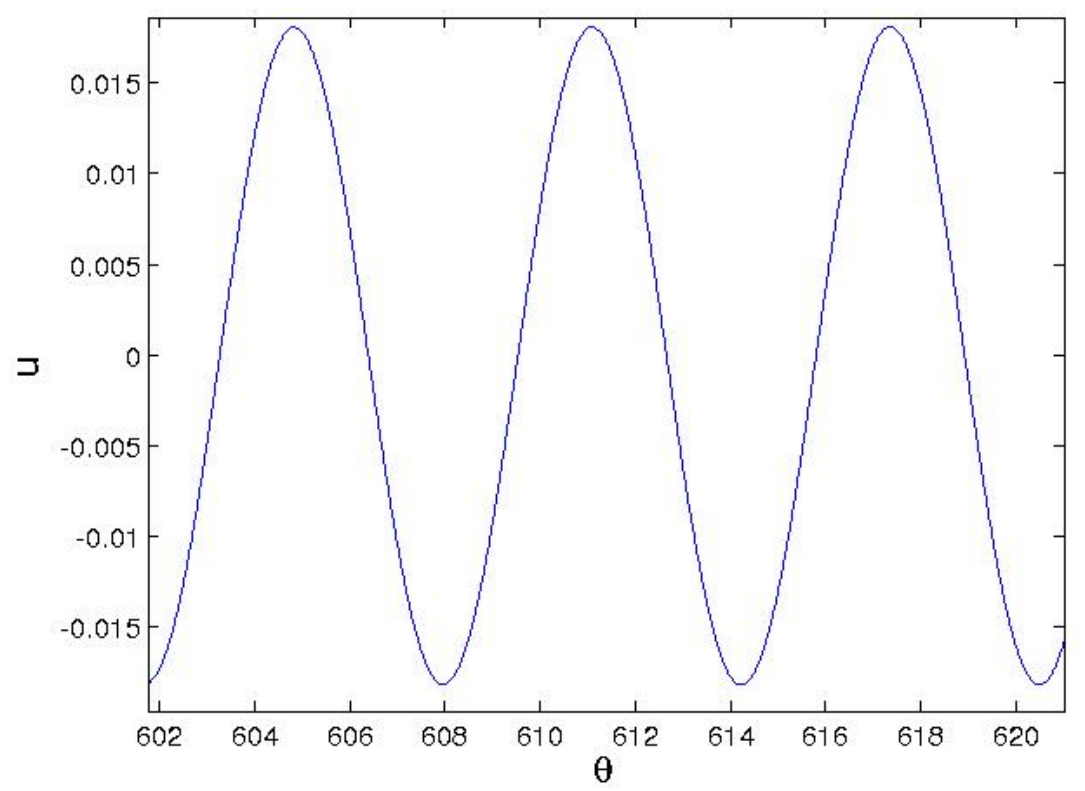

Figure 3.3: Plot of $u(x, \theta)$ as a function of $\theta$ for Case 3 at $x=0.5$. 


\section{Example 2: $n=3$}

In the previous example, we found that when $\alpha=0$, the response is unbounded. This, in turn, suggest that the odd nonlinearity form can lead to an unbounded response. To illustrate more of this result, we study in this example the same form of model problem in equation (3.3) with $f(u)$ in the form $u^{3} u_{x}$ and the boundary condition the same as in equation (3.4).

The new form of the model problem becomes

$$
\begin{gathered}
\lambda^{2} u_{\theta \theta}-u_{x x}+u^{3} u_{x}=0, \\
u(0, \theta)=m \sin (\theta),(\alpha-1) u(1, \theta)-\alpha u_{x}(1, \theta)=0 .
\end{gathered}
$$

Using the perturbation techniques as we did in Example 1, we obtain the same linear problem in equations (3.9) and (3.10), where the eigenvalue equation is given by $(3.13)$.

\section{Calculation of Amplitude A}

The first correction to linear theory in the perturbation solution to equations (3.30) and (3.32) (i.e., at $\left.O\left(\epsilon^{2}\right)\right)$ is given by

$$
\omega_{0}^{2} u_{2 \theta \theta}-u_{2 x x}=-2 \omega_{0} \omega_{1} u_{1 \theta \theta}
$$

and

$$
u_{2}(0, \theta)=0,(\alpha-1) u_{2}(1, \theta)-\alpha u_{2 x}(1, \theta)=0
$$

Notice that the problem of solving (3.32) subject to boundary conditions given in equation (3.33) is an inhomogeneous form of the problem (3.9) subject to (3.10). Since (3.9) has nontrivial solutions, then (3.32) can have a solution only if the right side satisfies appropriate orthogonality conditions. To show this, we shall consider a peri- 
odic solution $u(x, \theta)$ in $\theta$, and multiply both sides of equation (3.32) by $\sin \left(\omega_{0} x\right) \sin (\theta)$ then integrate with respect to $x$ from 0 to 1 and with respect to $\theta$ from 0 to $2 \pi$. We obtain

$$
0=-\frac{\pi}{2} \omega_{1}\left(2 \omega_{0}-\sin \left(2 \omega_{0}\right)\right)
$$

Hence, the value of $\omega_{1}$ is zero. Similarly, we may find $\omega_{2}, \ldots, \omega_{5}$ are also zero.

Based on this, the perturbation scheme given in (3.7) still works with the subsequent expansion for $\lambda$ :

$$
\lambda=\omega_{0}+\epsilon^{6} \delta+\cdots,
$$

where $\delta=\omega_{6}$ corresponds to a detuning from resonance.

Notice that the assumption that the input of $O\left(\epsilon^{3}\right)$ yields an output of $O(\epsilon)$ does not work here. However, we require the input of $O\left(\epsilon^{7}\right)$ to yield an output of $O(\epsilon)$, and the perturbation scheme should continue to $O\left(\epsilon^{7}\right)$ to obtain a condition for $A$ that involves $A^{7}$.

Using $\omega_{1}=0$ in equation $(3.32)$, it is clear that $u_{2}(x, \theta)$ must be in form $u_{2}(x, \theta)=$ $B \psi(x) \sin (\theta)$, where $\psi(x)$ satisfies the same equation as $\phi(x)$ given by equation (3.12). Therefore $u_{2}(x, \theta)=B \sin \left(\omega_{0} x\right) \sin (\theta)$.

We note that the amplitude of output depends on $n$ in the nonlinear form $u^{n} u_{x}$, which is crucial for the approximate analytic solution. In order to capture the balance on the amplitude $A$ in Example 1, where $n=1$ and the input is $O(m)$, we determine that the output should be $O\left(m^{\frac{1}{3}}\right)$; i.e., $m=\epsilon^{3}$. However, in Example 2, where $n=3$ and the input is also $O(m)$, we need to go higher to capture the nonlinearity and get resonance; in fact, the output for this case is $O\left(m^{\frac{1}{7}}\right)$. So, in general for the nonlinear form $u^{n} u_{x}$ where the input is $O(m)$, the output must be $O\left(m^{\frac{1}{2 n+1}}\right)$. 
The balance at $O\left(\epsilon^{7}\right)$ is of the form

$\omega_{0}^{2} u_{7 \theta \theta}-u_{7 x x}=-2 \omega_{0} \delta u_{1 \theta \theta}-\left(u_{2}^{3}+6 u_{1} u_{2} u_{3}+3 u_{1}^{2} u_{4}\right) u_{1 x}-\left(3 u_{1} u_{2}^{2}+3 u_{1}^{2} u_{3}\right) u_{2 x}-3 u_{1}^{2} u_{3 x}-u_{1}^{3} u_{4 x}$,

and

$$
u_{7}(0, \theta)=\sin (\theta),(\alpha-1) u_{7}(1, \theta)-\alpha u_{7 x}(1, \theta)=0
$$

After multiplying equation (3.36) by $u_{1}(x, \theta)$ and integrating both sides for $0 \leq x \leq 1$, $0 \leq \theta \leq 2 \pi$, then using the boundary condition in equation (3.37) as we did in the first correction gives the following restriction on $A$ :

$$
N A^{7}-A \delta \frac{\pi}{2}\left(2 \omega_{0}-\sin \left(2 \omega_{0}\right)\right)=\omega_{0} \pi
$$

where $N$ is a function of $\omega_{0}$.

For example, if we consider $\alpha=2$, we can find the eigenvalue by substituting in the eigenvalue equation given by(3.13). Then, by choosing $\delta=0$, we obtain from equation (3.38) the amplitude $A=3.477436544$. However, we will use this result when comparing the numerical solution with the leading-order approximation.

Now, we examine the following cases.

Case 1: $\alpha=0$.

Consequently, the eigenvalue equation (3.13) becomes $\tan \left(\omega_{0}\right)=0$. Thus the eigenvalues are commensurate and given by $\omega_{0}=\lambda_{n}=n \pi$, where $n \geq 1$.

For the purpose of numerical approximation and since we have higher order of nonlinearity, we expect a smaller response. Therefore we let $m=0.01$. Applying the Runge-Kutta method of order four with the boundary conditions $u(0, \theta)=m \sin (\theta)$ and $u(1, \theta)=0$, we obtain the following result: 


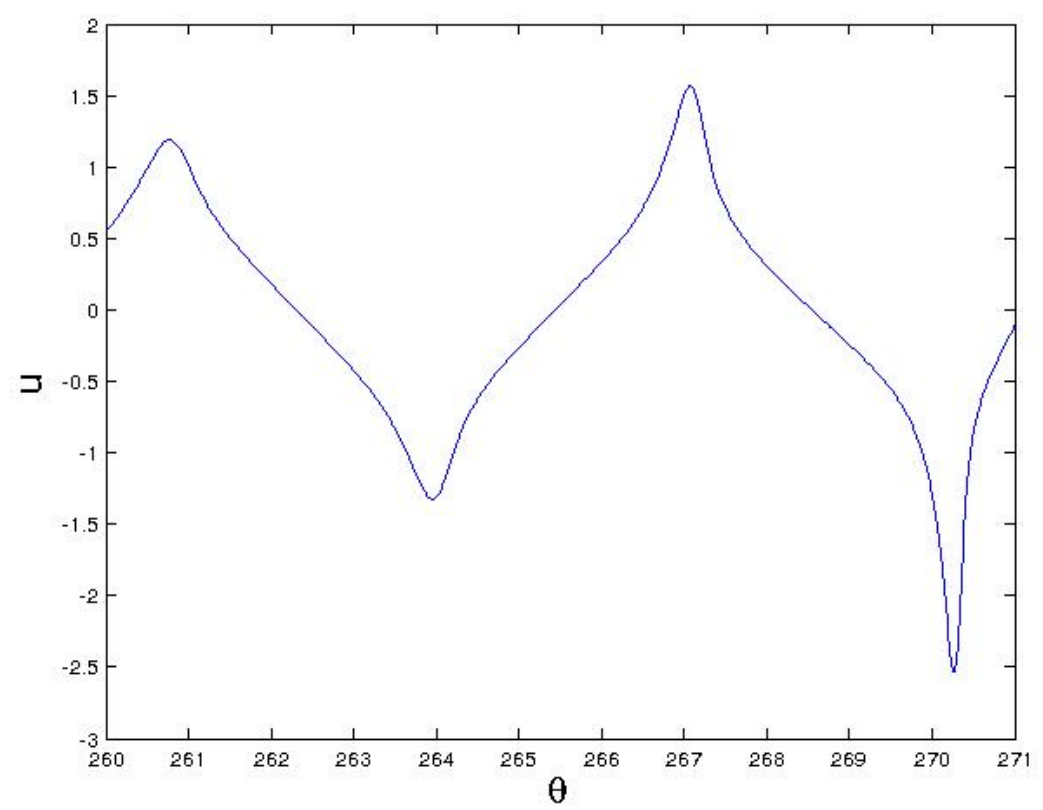

Figure 3.4: Plot of $u(x, \theta)$ as a function of $\theta$ for case 1 at $x=0.5$.

Figure 3.4 shows the exact numerical solution for $\alpha=0$, and it suggests that the response is unbounded. This is also consistent with Case 1 in Example 1 when $n=1$. Therefore we can note that the existence of unbounded solutions depends on the form of nonlinearity $u^{n} u_{x}$. In particular, if $n$ is odd, then we obtain unbounded solutions. Also, it explains that there is a correlation between the unbounded regime and the commensurate spectrum. 
Case 2: $\alpha=2$.

In this case, we obtain an incommensurate eigenvalue, which is similar the Case 2 in Example 1. Following the perturbation approach as discussed above, the approximation solution yields a continuous response. The result of comparison between the continuous numerical solution and the leading-order perturbation solution is shown in Figure 3.5.

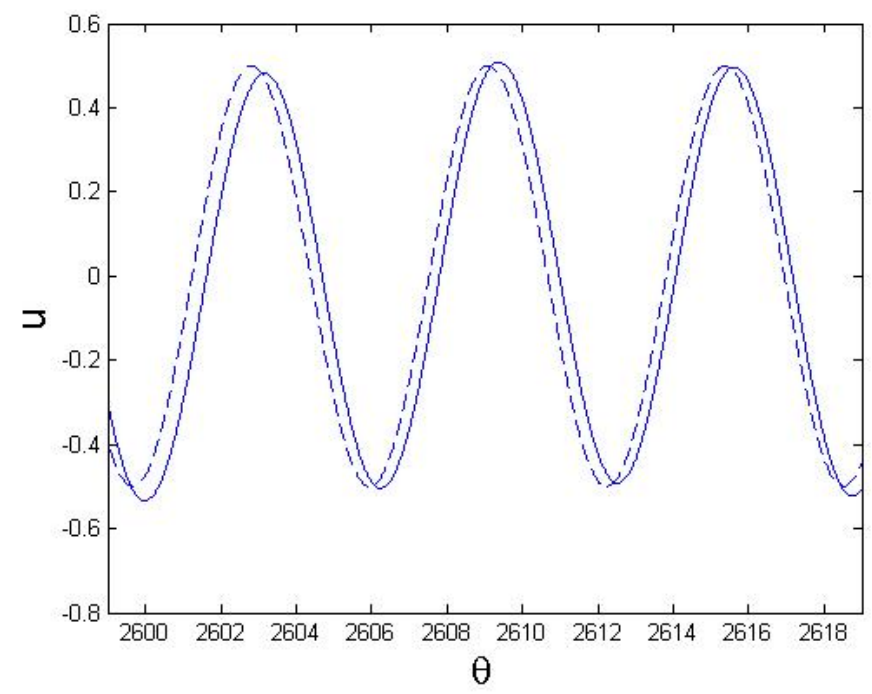

Figure 3.5: Comparison of numerical solution (solid line) to leading-order approximation (dashed line) with $\alpha=2$.

\subsubsection{The generalized result for $f(u)=u^{n} u_{x}$}

We showed that, for $n=1$ in Example 1 and after studying the case $\alpha=0$, the behavior of the solution is unbounded. However, for $n=3$, as in Example 2, we see that, for the same value of $\alpha$, there is also an unbounded response. Therefore this suggests that, for the model problem given by equation (3.5), the existence of an unbounded responses depends on the form of the nonlinearity, in addition to the commensurate nature of the resonant spectrum. To demonstrate this, we consider two further examples for $n=4$ and for $n=5$. We consider now only the case $\alpha=0$. 
The exact numerical solutions with the nonlinearity forms $u^{4} u_{x}$ and $u^{5} u_{x}$ are shown in Figure 3.6.

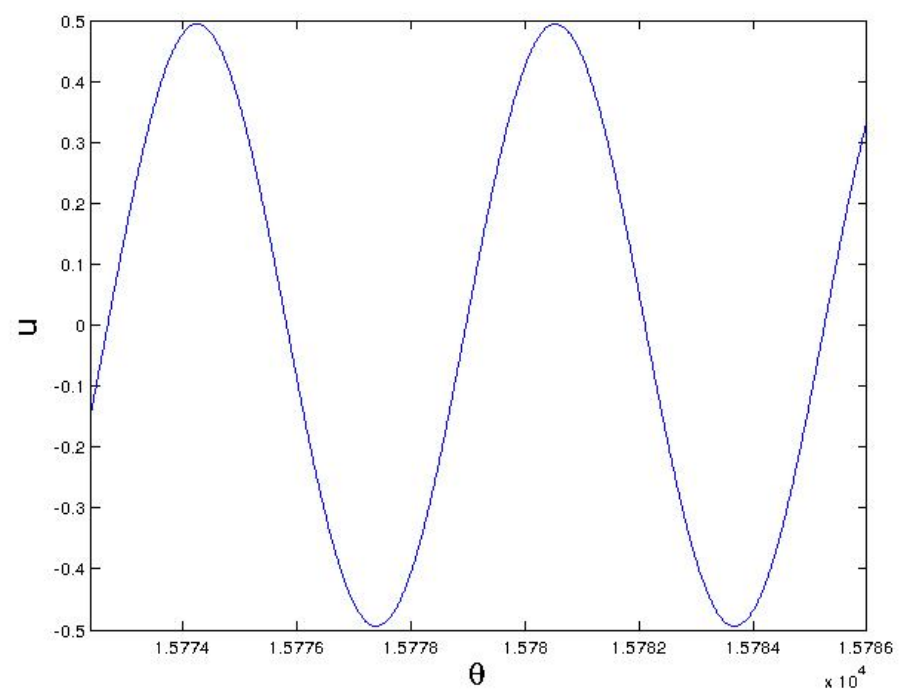

(a)

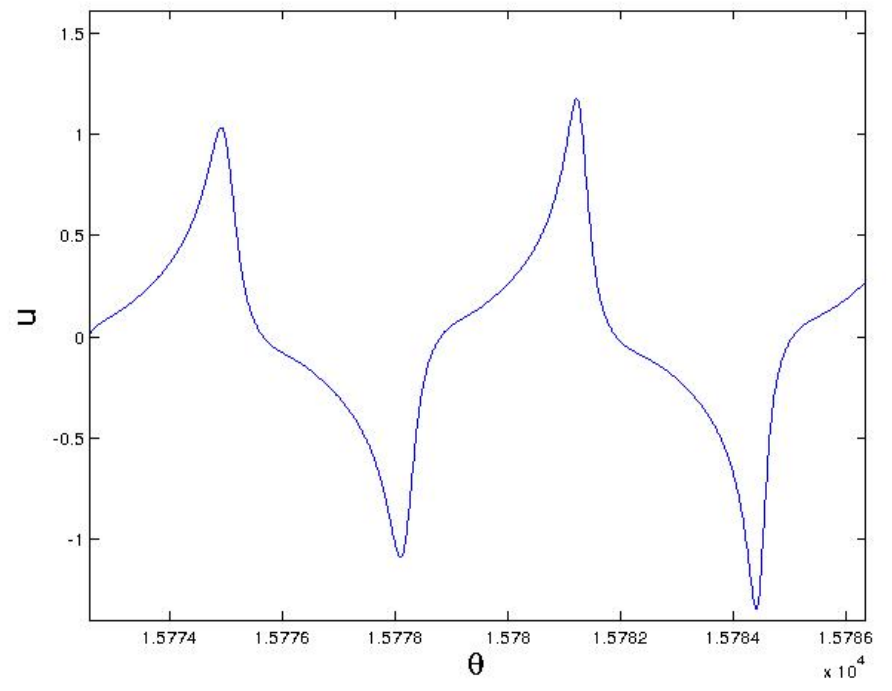

(b)

Figure 3.6: Plots solution of model problem $(3.5), u(x, \theta)$ as a function of $\theta$ with different form of nonlinearity with $\alpha=0$ at $x=0.5$. In (a) it is shown the result for $f(u)=u^{4} u_{x}$ while in (b) it is shown those obtained for $f(u)=u^{5} u_{x}$.

We close this subsection by noting that, for the nonlinear model problem given by equation (3.5), the behaviour of the solution depends on the power $n$. In other words, the results suggest that, when $n$ is odd, we obtain an unbounded response as shown in Examples 1 and 2. The commensurable of the eigenvalues is a significant element 
for the existence of unbounded solution. But if $n$ is even, then we have the continuous response.

Notice that the nonlinearity form $u u_{x}$ can be written in the form $\left(\frac{u^{2}}{2}\right)_{x}$, and $u^{4} u_{x}$ as $\left(\frac{u^{5}}{5}\right)_{x}$. This is especially relevant to the nature of the underlying nonlinearity function $F(U)$ in the conservation law form, $U_{t}+F(U)_{x}=0$. For more on hyperbolic equations, see $[3,20,39,41,51]$. So, in case of even, like $\left(\frac{u^{2}}{2}\right)_{x}$, where is unbounded response. However, when the nonlinearity is odd, as $\left(\frac{u^{5}}{5}\right)_{x}$, we get a continuous response. Therefore the term $u^{n} u_{x}$ in the model problem given by equation (3.5) can be written as a nonlinearity function $F(U)=\frac{u^{n+1}}{n+1}$ in terms of conservation form. Then the even yield unbounded response, but the odd yields a continuous response.

In general, we should notice that, for the even case, the resonance occurs at $O\left(\epsilon^{n+1}\right)$, while, for the odd case, the resonance arises at $O\left(\epsilon^{2 n+1}\right)$, provided $(n+1) \lambda$ is not an eigenvalue, since the eigenvalue are assumed incommensurate. A summary of the behaviour of model problems in the form given by equation (3.5) with varying the expression of nonlinearity is presented in Table 3.1.

\begin{tabular}{|c|c|c|}
\hline$f(u)$ & Value of $\alpha$ & Behavior of the solution \\
\hline$u u_{x}$ & 0 & Unbounded \\
& 2 & Continuous \\
\hline$u^{3} u_{x}$ & 0 & Unbounded \\
& 2 & Continuous \\
\hline$u^{4} u_{x}$ & 0 & Continuous \\
$u^{5} u_{x}$ & 0 & Unbounded \\
\hline
\end{tabular}

Table 3.1: Performance summary of the nonlinear types of model problem (3.5). 


\subsection{Nonlinearity Form $f(u)=u^{2}$}

In this example, we consider another nonlinearity:

$$
\begin{gathered}
u_{t t}-u_{x x}+u^{2}=0, \\
u(0, t)=m \sin (\lambda t),(\alpha-1) u(1, t)=\alpha u_{x}(1, t) .
\end{gathered}
$$

Substituting $\theta=\lambda t$ into (3.39) and (3.40) yields

$$
\lambda^{2} u_{\theta \theta}-u_{x x}+u^{2}=0,
$$

where the boundary conditions are still given by equation (3.4).

The model problem given by (3.39) can be written in the form of Klein-Gordon equation; i.e., $u_{t t}-a u_{x x}+b u+k u^{n}$, where). A number of studies have been provided solutions, for example Wazwaz in 2005 obtained many travelling wave solutions with different physical structure by using tanh method and Sine-cosine method.

Applying the same perturbation technique as we discussed in Section 3.1, we obtain the same solution of $\phi(x)$ that is given in equation (3.12). After using the boundary conditions, we see that the conditions for the function $\phi(x)$ and the eigenvalue equation are respectively given by equations (3.11) and (3.13).

Following the analytic approach discussed in Section 3.1, we can obtain the amplitudefrequency relation of nonlinearity form $f(u)=u^{2}$ with any $\alpha$. For example, when $\alpha=2$, we get

$$
-0.45389 A^{3}-14.1274 A \delta-\lambda_{0} \pi=0 .
$$

By choosing $\delta=0$ and substituting the corresponding fundamental frequency $\lambda_{0}$, we 
determine the amplitude $A$ at the leading-order approximation for $u(x, \theta)$ is $A=$ -3.17042 . Now, we study the following cases of the problem.

Case 1: $\alpha=0$.

Therefore the eigenvalues can be determined by the eigenvalue equation (3.13) and have the form $\lambda_{n}=n \lambda_{1}$, where $\lambda_{1}=\pi$ and $n \geq 2$. Once again, the results of this case are commensurate eigenvalues. Setting $\alpha=0$ and satisfying the boundary conditions given in equation (3.40) with the same method used in previous cases, we obtain the following result. By comparing with Case 1 in Example 1, we notice that this case

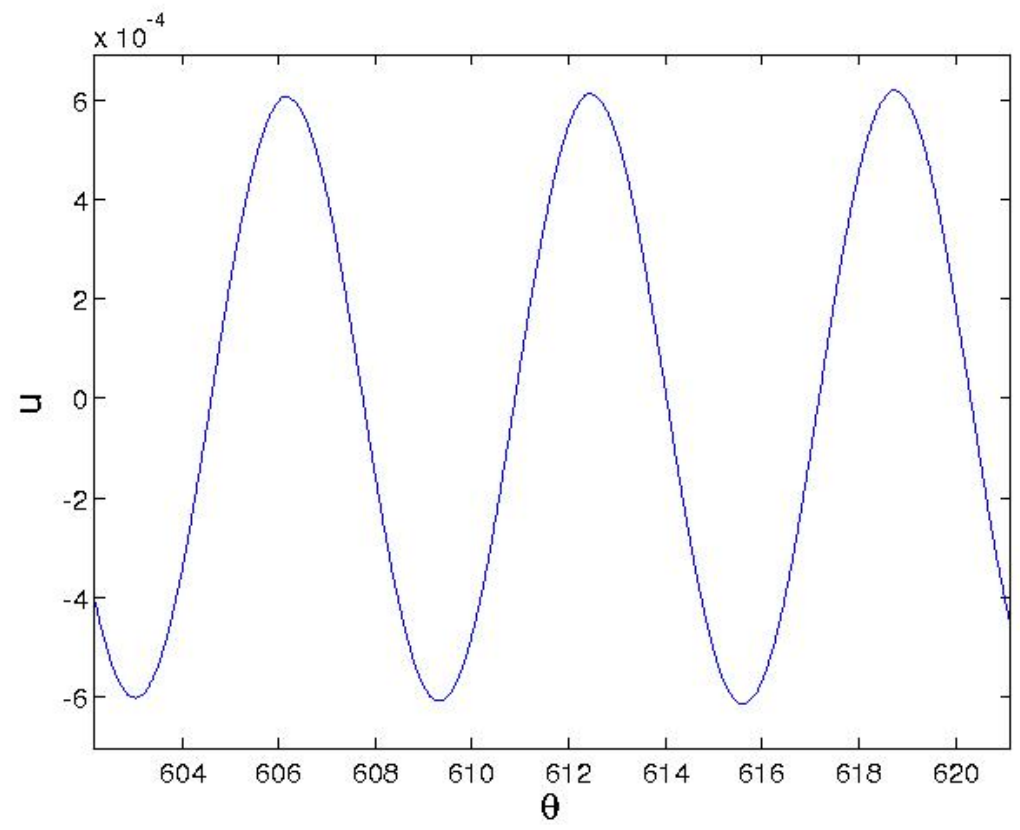

Figure 3.7: Plot of $u(x, \theta)$ as a function of $\theta$ for Case 1 and the continuous response at $x=0.5$.

leads to a continuous response, whereas there is an unbounded response in Example 1. These results suggest that, while the eigenvalue spectrum is necessary, the form of the nonlinearity also plays a key role in the qualitative nature of the output.

Case 2: $\alpha=2$.

We can choose any value of $\alpha$ such that $0<\alpha<\infty$, but for the purpose of numer- 
ical simulation, we set $\alpha=2$. The continuous solution is obtained by perturbation techniques as illustrated in the previous example and compared with that found by numerical methods. Applying the boundary conditions given in equation (3.40) and using the same method with $\alpha=2$ and $m=0.0001$, we obtain the numerical solution.

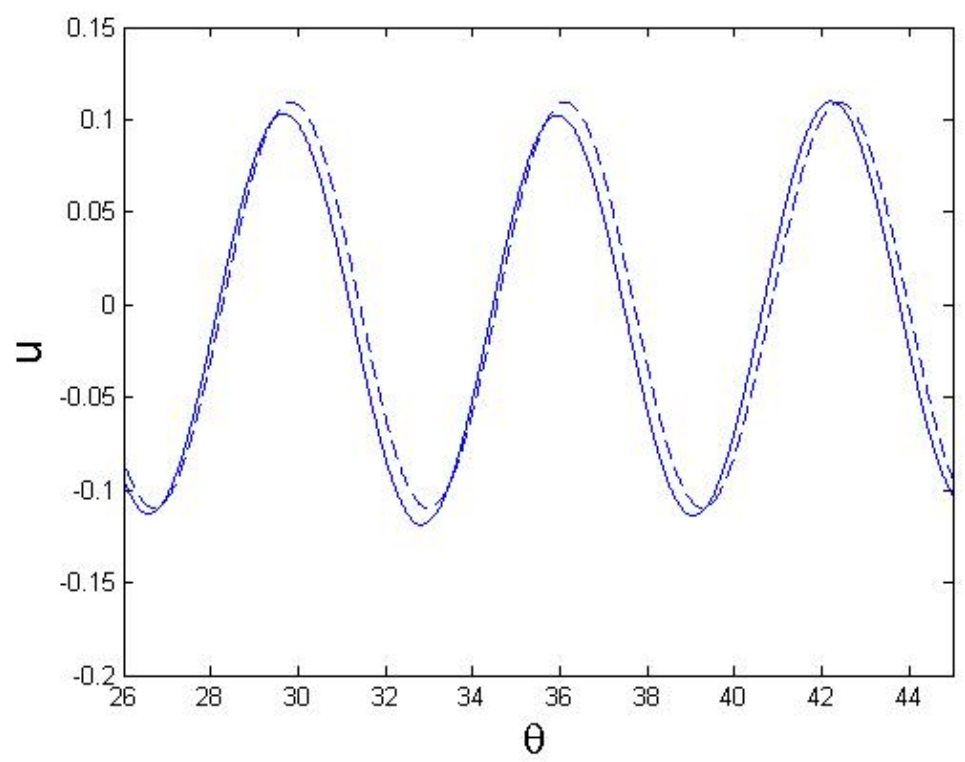

Figure 3.8: Comparison of numerical solution (solid line) to leading-order approximation (dashed line) with $\alpha=2$, and the solution of (3.42).

In order to obtain a comparison between the numerical simulation and the analytical solution, we apply the classical Runge-Kutta method with steady-state result. Figure 3.8 compares the continuous numerical solution for the Case $\alpha=2$ with leadingorder perturbation solution for $u(x, \theta)$. Again, we find a good agreement, and we can see there are small oscillations around the leading-order approximation from the numerical results.

Case 3: $\alpha=1$.

The result of solving the eigenvalue equation for $\lambda$ provided us the same form of the eigenvalues as in Case 3 in Example 1, which is again commensurate. Choosing $m=0.0001$ and using a similar method in the previous example with the boundary condition $u_{x}(1, \theta)=0$, we find that the approximation solution achieves a continuous 
response, and the result is shown in Figure 3.9 below.

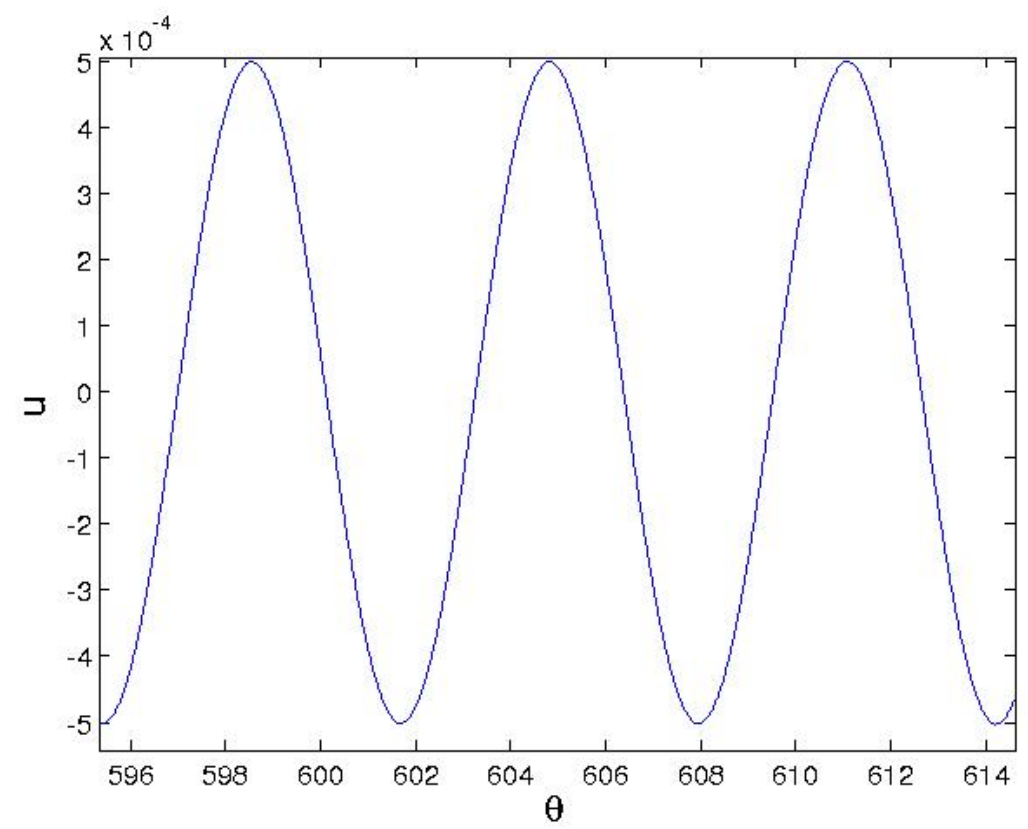

Figure 3.9: Plot of $u(x, \theta)$ as a function of $\theta$ for Case 3 showing a continuous response at $x=0.5$.

We should notice that all cases of $\alpha$ with the term $f(u)=u^{2}$ yield continuous responses only, and thus, as we discussed before, were now the nonlinearity is $F(U)=$ $\int u^{2} d x$, which is nonlocal; hence unbounded solutions cannot be expected.

In the next section, we will study more examples to support how the effect of nonlinearity plays a major role in the type of the outcome responses.

\subsection{Other Nonlinear Forms}

A generalization from the previous work may be stated as follows: the detailed representation of nonlinear terms, $f(u)$ in equation (3.1), is necessary to understand the behaviour of the solution and existence of an unbounded response in an effective and successful way. In the following two figures, we will study some other cases of nonlinearity in the derivative and trigonometric nonlinearity respectively. Figure 3.10 
shows the results with $f(u)=u_{x}^{2}$ and $f(u)=u_{x}^{3}$ and their role in the behaviour of solutions. Also, from Figure 3.11, we observe how a trigonometric nonlinearity term plays a role in the nature of the output.

We should note that when the model problem has the nonlinearity term $f(u)=\sin (u)$, the form of nonlinear wave equations is similar to the Sine-Gordan equation, which has many applications in physics and applied mathematics. There are many methods can be used to obtain the solution of the Sine-Gordan equation, where it may include solitary pattern, or come as a periodic solution. For more details, we refer to [[69], [4]].

As an example that helps us to understand the effect of nonlinearity in the character of the outcome, we also consider the model problem in equation (3.1) with $f(u)$ of the form $g(u) u_{x}$, where $g(u)$ is a trigonometric function.

Then, in terms of conversation laws $U_{t}+G(U)_{x}=0$, we define the function $G(u)=$ $\int g(u) d u$. In order to explain how the function $G(u)$ plays a key role in the behaviour of the outcome, whether it is continuous or unbounded, we introduce the two examples in Figure 3.12, one with $g(u)=\sin (u)$, and the other with $g(u)=\cos (u)$. It can be noticed from Figure 3.12 that if $G(u)$ is an even function, then there is an unbounded outcome, while if it is an odd function, ther is a continuous response.

A summary of the behaviours of model problems in the form given by equation (3.1) with many types of nonlinearity term $f(u)$ such as nonlinearity of derivative and trigonometric nonlinearity is presented in Table 3.2.

\begin{tabular}{|c|c|}
\hline$f(u)$ & Behaviour of the solution \\
\hline $\sin u$ & Continuous \\
$\cos u$ & Continuous \\
\hline$u_{x}^{2}$ & Continuous \\
$u_{x}^{3}$ & Unbounded \\
\hline $\cos u u_{x}$ & Continuous \\
$\sin u u_{x}$ & Unbounded \\
\hline
\end{tabular}

Table 3.2: Performances summary of the nonlinear types of model problem (3.1). 


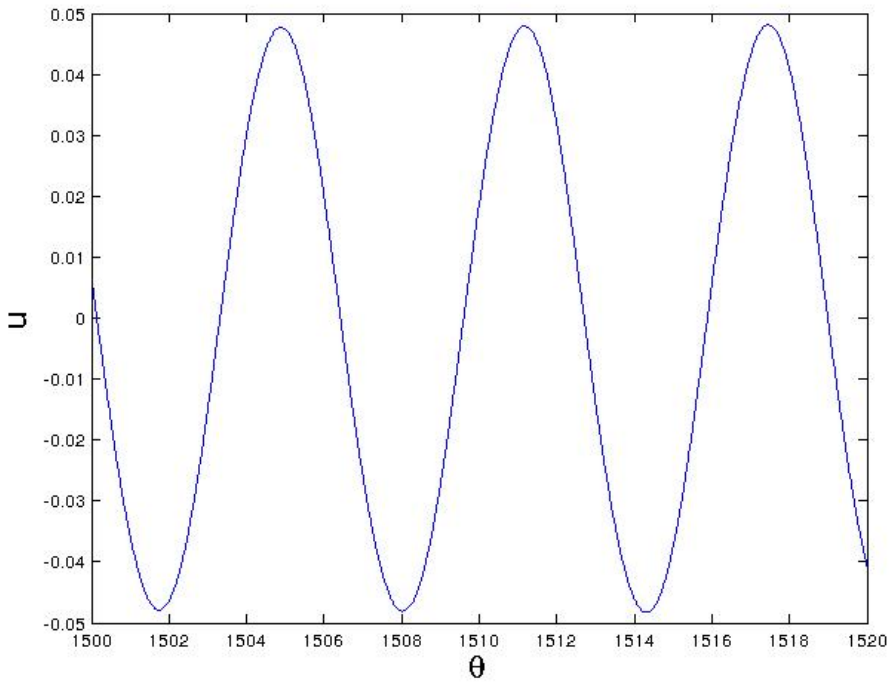

(a)

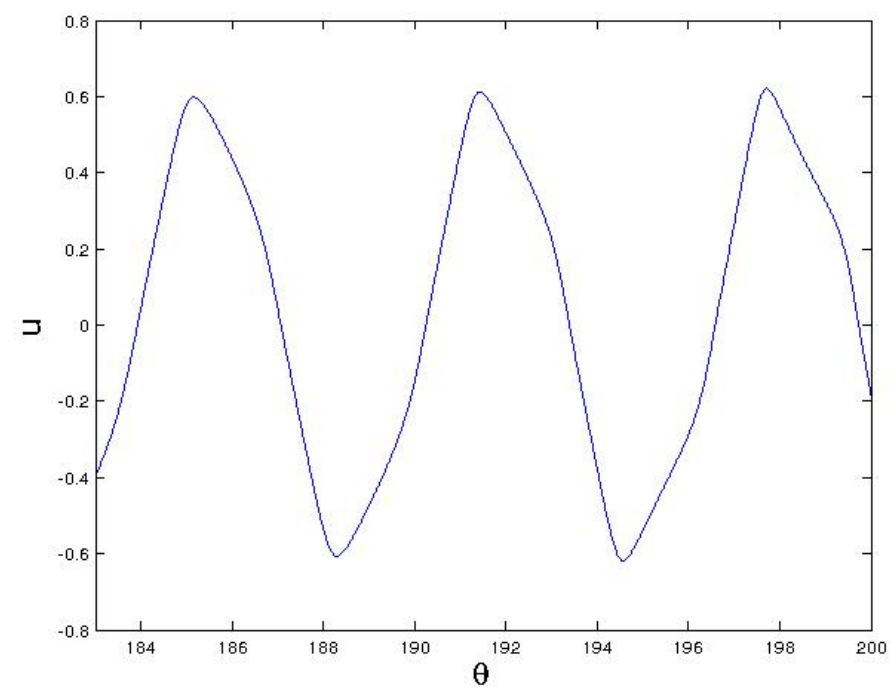

(b)

Figure 3.10: Plots of the solution of model problem $(3.1), u(x, \theta)$ as a function of $\theta$ with nonlinear derivatives at $x=0.5$. In (a) it is shown the result for $f(u)=u_{x}^{2}$ while in (b) it is shown those obtained for $f(u)=u_{x}^{3}$. 


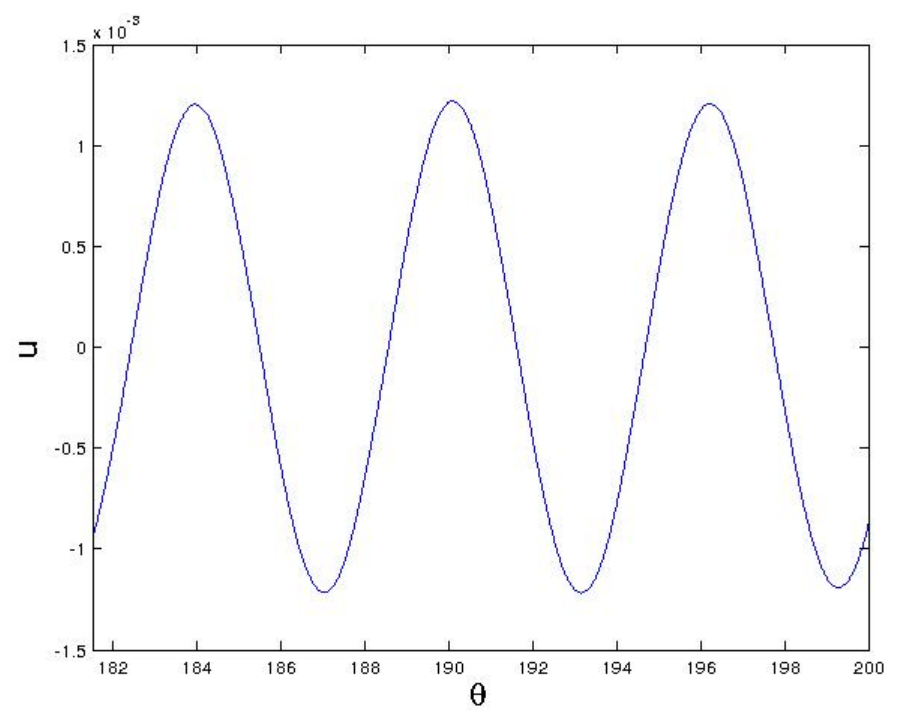

(a)

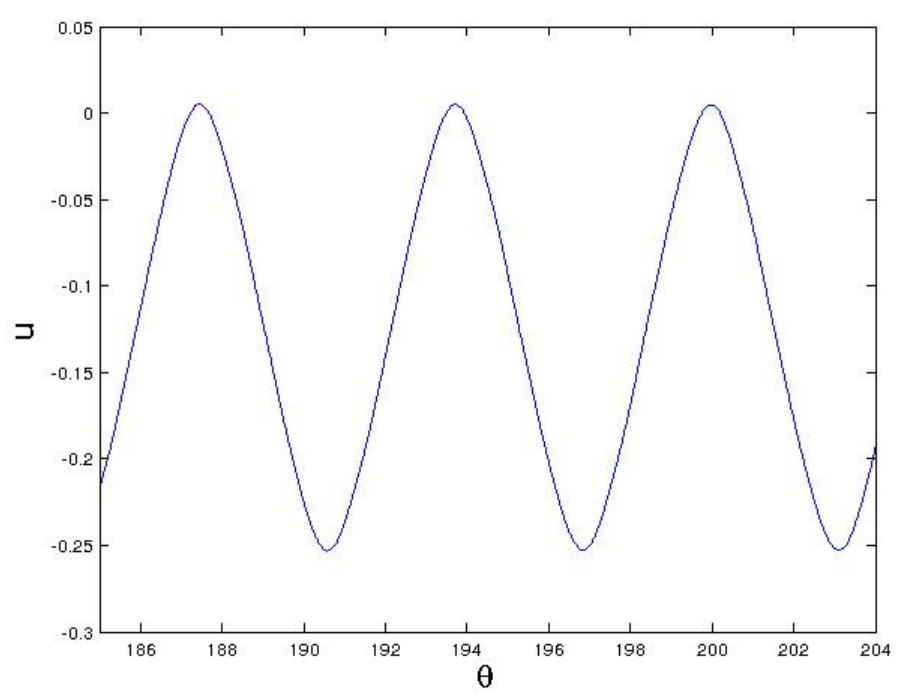

(b)

Figure 3.11: Plots of the solution of model problem (3.1), $u(x, \theta)$ as a function of $\theta$ with trigonometric nonlinearity at $x=0.5$. In (a) it is shown the result for $f(u)=$ $\sin (u)$ while in (b) it is shown those obtained for $f(u)=\cos (u)$. 


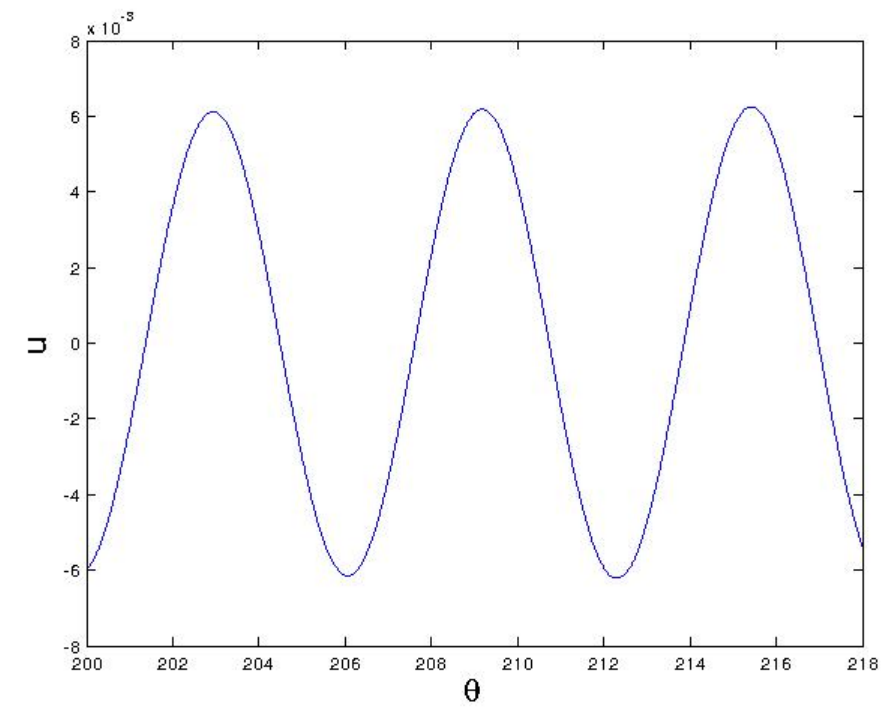

(a)

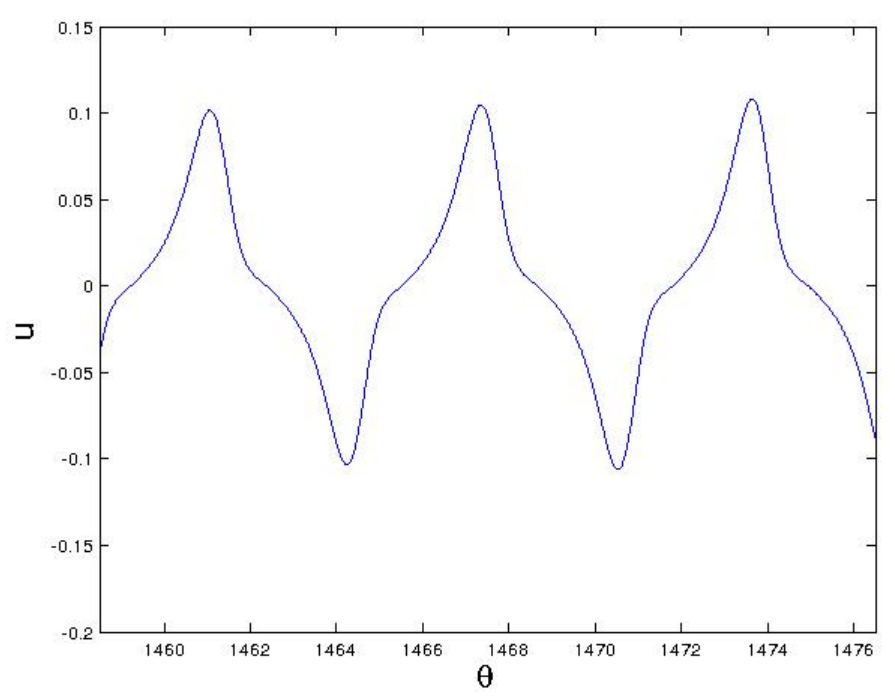

(b)

Figure 3.12: Plots of the solution of model problem (3.1), $u(x, \theta)$ as a function of $\theta$ with trigonometric and derivative nonlinearity terms at $x=0.5$. In (a) it is shown the result for $f(u)=\cos (u) u_{x}$ while in (b) it is shown those obtained for $f(u)=\sin (u) u_{x}$. 


\subsection{Summary}

In this Chapter, the behaviour of the model problem in the form given by equation (3.1) with a different type of nonlinearity term, $f(u)$, was discussed.

First, we focused mostly on the existence of unbounded response by discussing many cases of the nonlinearity term $u^{n} u_{x}$. We obtained that the nature of outcome response depends on the power $n$. In particular, we determined that, when $n$ is even, we have a continuous response. But if $n$ is odd, we obtain an unbounded response.

Next, we carried out the numerical solution for different types of nonlinearity form such as nonlinearity of derivative and trigonometric nonlinearity. As a result, the form of nonlinearity is a critical element for the existence of an unbounded response and the transition between the two acoustic regimes: continuous and unbounded.

The natural question arising from the previous results is: what about a general nonlinearity term? Table 3.3 summarizes the nonlinear model problem with $f(u)=$ $(1-\beta) u^{2}+\beta u u_{x}$, where $\beta \in[0,1]$. Notice that, when $\beta=0$, the nonlinearity term becomes $u^{2}$, and this corresponds to the function $F(U)=\int u^{2} d x$. For this case, we conclude that there exists a continuous response as discussed in Section 3.2. But if $\beta=1$, the form of nonlinearity is $u u_{x}$, which is related to the even nonlinearity function $F(U)=\left(\frac{u^{2}}{2}\right)_{x}$, which yields a local unbounded response as in Example 1 . However, as seen in Table 3.3, if the value of $\beta$ is close to 1 , there is an unbounded response, and if $\beta$ is close to $\beta=0$, we obtain a continuous response. Figure 3.13 illustrates the transition between continuous and unbounded solutions.

So for the nonlinear form $u^{n} u_{x}$, we define the function $F(U)=\frac{u^{n+1}}{n+1}$. Then the even nonlinearity function (i.e., $F(U)$ ) yields an unbounded response, but the odd yields a continuous response.

In general, based on the results in this chapter, if we consider the nonlinear term of 
our model problem as a function with a different nonlinearity term, $f\left(u, u_{x}\right)$, which is continuous and differentiable (e.g., $\left.f\left(u, u_{x}\right)=a_{0}+a_{1} u+a_{2} u_{x}++a_{3} u^{2}+a_{4} u u_{x}+a_{5} u_{x}^{2}\right)$, then, as we saw in this chapter, most cases yield a continuous response, and only specific cases, when there is a local and even nonlinearity, will lead to an unbounded response.

\begin{tabular}{|c|c|c|c|}
\hline$\beta$ & $\mathrm{b}$ & $\epsilon$ & Behaviour of the solution \\
\hline 0 & $500 \pi$ & 0.0001 & Continuous \\
\hline 1 & $1000 \pi$ & 0.0001 & Unbounded \\
\hline 0.01 & $1000 \pi$ & 0.0001 & Continuous \\
\hline 0.0001 & $1000 \pi$ & 0.0001 & Continuous \\
\hline 0.5 & $1000 \pi$ & 0.01 & Unbounded \\
\hline 0.8 & $1000 \pi$ & 0.0001 & Unbounded \\
\hline 0.6 & $1000 \pi$ & 0.0001 & Unbounded \\
\hline 0.9 & $1000 \pi$ & 0.0001 & Unbounded \\
\hline
\end{tabular}

Table 3.3: Performance summary of the nonlinear types of model problem (3.1) with $f(u)=(1-\beta) u^{2}+\beta u u_{x}$. 


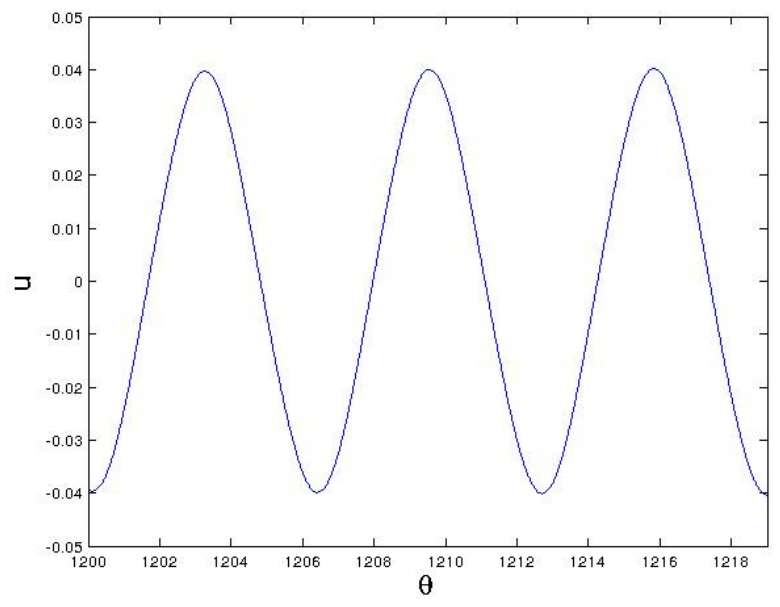

(a)

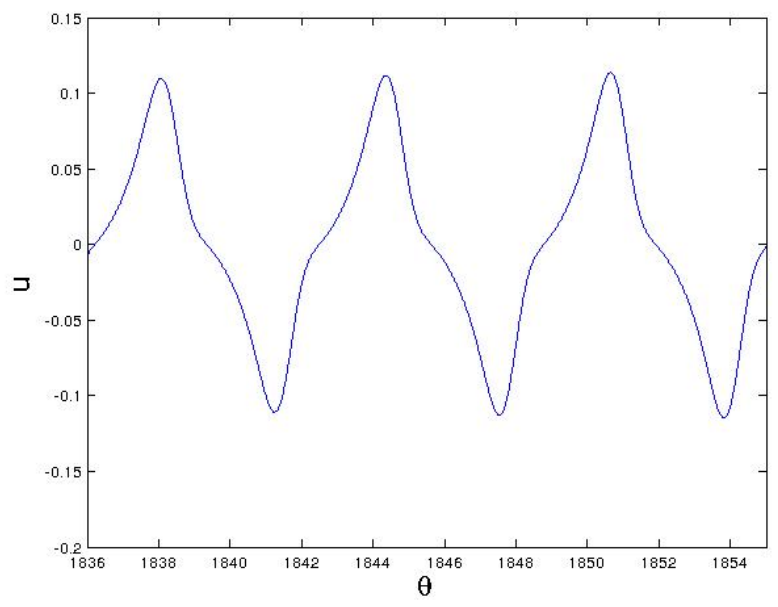

(b)

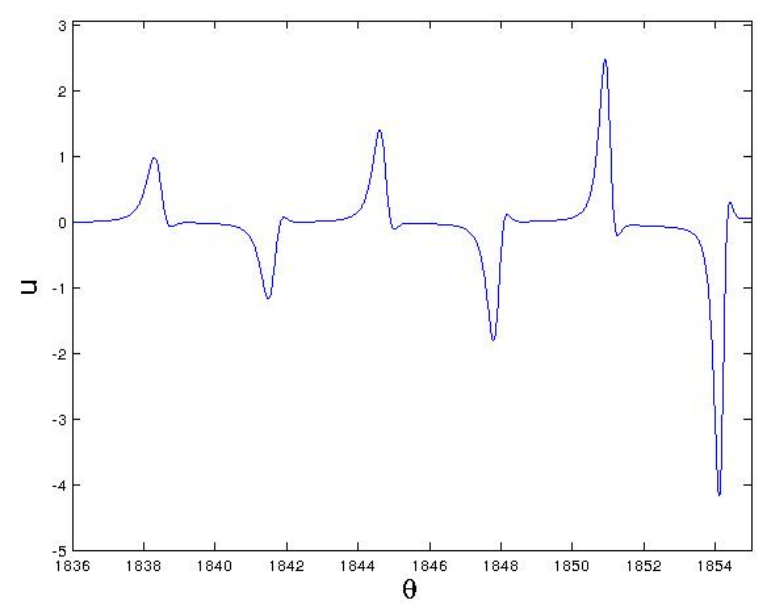

(c)

Figure 3.13: Effect of nonlinearity parameter $\beta$ on the transition between unbounded and continuous solutions for the model problem (3.3), the result for $f(u)=(1-$ $\beta) u^{2}+\beta u u_{x}$, and $\beta=0.4,0.6,0.8$ (a-c). 


\section{Chapter 4}

\section{Nonlinear Wave Resonance with Damping}

As discussed in the previous chapter, the nonlinear form of the wave problems and the commensurate nature of the spectrum play a crucial role in the resonant response, and in particular whether it is continuous or unbounded. Many studies have dealt with analytical solutions of nonlinear wave problems (e.g., Kevorkian [30] and Whitham [67]). In this chapter, we study a nonlinear wave-resonant model problem that captures the key features while incorporating damping effects and explain how this affects the qualitative nature of the resonant output. For useful references on resonant oscillation with damping see, $[13,58]$. Then we obtain the analytic solution, where the leading-order includes a single mode or multiple modes. In Section 4.1, we formulate the nonlinear model problem and describe the boundary conditions. In section 4.2, we investigate the linear and nonlinear theory analytically, where multiple modes at leading-order are now required for some cases, as we will discuss later. Finally, we compare the numerical solutions with the analytical predictions. 


\subsection{Formulation of the Model Problem}

In this section, we will study the same model problem that we studied in Chapter 3, but the difference is that now we introduce a damping coefficient $\nu$.

Consider the following damped nonlinear model problem

$$
\lambda^{2} u_{\theta \theta}-u_{x x}+u u_{x}=\lambda^{2} \nu \epsilon^{2} u_{x x \theta}
$$

where $\nu$ is the damping coefficient that is considered to be of order $O(1)$, over the domain $x \in[0,1]$ with a periodic function $u(x, \theta)$ in $\theta$.

The boundary conditions are

$$
u(0, \theta)=\epsilon^{3} \sin (\theta), \quad(1-\alpha) u(1, \theta)+\alpha u_{x}(1, \theta)=0,
$$

where the first boundary condition represents the forced boundary at $x=0$, and the second one at $x=1$ corresponds to a fixed boundary.

\subsection{Analytic Approximation Using a Single Mode}

In this section, we obtain the analytic solution for the damped nonlinear model problem. We consider here the case where there is only one mode in the leading-order approximate solution. Then we explain how the solvability condition is obtained using the perturbation technique together with another condition.

We start by carrying out the weakly nonlinear solution using the perturbation method for $\epsilon \ll 1$, where the solution is written as a perturbation series in powers of $\epsilon$. We 
consider the following perturbation expansions:

$$
\begin{aligned}
u(x, \theta) & =\epsilon u_{1}(x, \theta)+\epsilon^{2} u_{2}(x, \theta)+\epsilon^{3} u_{3}(x, \theta)+\cdots, \\
\lambda & =\lambda_{0}+\epsilon^{2} \delta+\epsilon^{3} \lambda_{3}+\cdots
\end{aligned}
$$

where $\left|u_{i}\right|=O(1), i=1,2,3, \ldots, \lambda_{0}$ corresponds to the fundamental resonant frequency and $\delta=\lambda_{2}$. We should notice that the second term in the expansion of $\lambda$ would be expected to be $\epsilon \lambda_{1}$, but it disappears because $\lambda_{1}=0$ in the same way as was shown in Example 1 of Chapter 3. We substitute expansions (4.3) and (4.4) into the nonlinear model problem in equation (4.1) and equate terms of $O(\epsilon)$ and $O\left(\epsilon^{2}\right)$.

At $O(\epsilon)$, we find that $u_{1}(x, \theta)$ satisfies the equation

$$
\lambda_{0}^{2} u_{1 \theta \theta}-u_{1 x x}=0
$$

with

$$
u_{1}(0, \theta)=0, \quad(1-\alpha) u_{1}(1, \theta)+\alpha u_{1 x}(1, \theta)=0 .
$$

Now, with damping included, let the leading-order solution $u_{1}(x, \theta)$ be in the form

$$
u_{1}(x, \theta)=\phi(x)(A \sin \theta+B \cos \theta)
$$

where $A$ and $B$ are arbitrary amplitudes to be determined later through the solvability conditions at $O\left(\epsilon^{3}\right)$. Here $\phi(x)$ is the eigenfunction corresponding to the fundamental eigenvalue $\lambda_{0}$. Substituting the second derivatives of $u_{1}$ with respect to $x$ and $\theta$ in equation (4.5) implies $\phi(x)$ satisfies the same eigenvalue problem given in equation (3.11).

According to the classical solution of $\phi(x)$ that is given in equation (3.12), we can 
write the eigenvalue equation as

$$
(\alpha-1) \sin \left(\lambda_{0}\right)=\alpha \lambda_{0} \cos \left(\lambda_{0}\right)
$$

Thus the nature of the underlying linear spectrum and whether it is commensurate

or incommensurate depends on the value of $\alpha$ as we will discuss in this chapter; e.g., when $\alpha=1$, then the eigenvalues are commensurate.

Therefore the leading-order solution is given by

$$
u_{1}(x, \theta)=\sin \left(\lambda_{0} x\right)[A \sin (\theta)+B \cos (\theta)]
$$

From this equation, it is clear that, for the linear solution (i.e., at $O(\epsilon)$ ), it only remains to obtain amplitudes $A$ and $B$. So we need to compute the nonlinear terms up to $O\left(\epsilon^{3}\right)$.

\section{Solvability conditions for the amplitudes $A$ and $B$}

At $O\left(\epsilon^{2}\right)$, the equations to determine $u_{2}(x, \theta)$ now becomes

$$
\lambda_{0}^{2} u_{2 \theta \theta}-u_{2 x x}=-u_{1} u_{1 x}
$$

and

$$
u_{2}(0, \theta)=0, \quad(1-\alpha) u_{2}(1, \theta)+\alpha u_{2 x}(1, \theta)=0 .
$$

By substituting the linear solution $u_{1}$ given by (4.9) into the right-hand side of equation (4.10), it is clear that the form of $u_{2}(x, \theta)$ must be

$$
u_{2}(x, \theta)=C_{3}(x)+C_{4}(x) \cos (2 \theta)+C_{5}(x) \sin (2 \theta) .
$$

After using this in equation (4.10) and grouping the terms involving the trigonometric 
functions, $\cos (2 \theta)$ and $\sin (2 \theta)$ respectively, we find that $C_{3}(x), C_{4}(x)$ and $C_{5}(x)$ satisfy the following equations :

$$
\begin{aligned}
-C_{3}^{\prime \prime}(x)+\frac{\lambda_{0}}{4}\left(B^{2}+A^{2}\right) \sin \left(2 \lambda_{0} x\right) & =0, \\
C_{4}^{\prime \prime}(x)+4 \lambda_{0}^{2} C_{4}(x) & =\frac{\lambda_{0}}{4} \sin \left(2 \lambda_{0} x\right)\left(B^{2}-A^{2}\right), \\
C_{5}^{\prime \prime}(x)+4 \lambda_{0}^{2} C_{5}(x) & =\frac{\lambda_{0}}{2} A B \sin \left(2 \lambda_{0} x\right),
\end{aligned}
$$

where the boundary conditions are

$$
C_{i}(0)=0, \quad(1-\alpha) C_{i}(1)+\alpha C_{i}^{\prime}(1)=0 i=3,4,5 .
$$

We should note that the solutions of the functions $C_{3}, C_{4}, C_{5}$ can be determined provided $2 \lambda_{0}$ is not an eigenvalue.

After solving each equation at (4.13) with corresponding boundary conditions in (4.14) for $C_{3}(x), C_{4}(x)$ and $C_{5}(x)$, we have

$$
\begin{aligned}
C_{3}(x)= & \frac{\left(A^{2}+B^{2}\right)}{16 \lambda_{0}}\left[-\sin \left(2 \lambda_{0} x\right)+2 \lambda_{0} \alpha x \cos \left(2 \lambda_{0}\right)+x(1-\alpha) \sin \left(2 \lambda_{0}\right)\right], \\
C_{4}(x)= & \frac{\left(B^{2}-A^{2}\right)}{32 \sin \left(2 \lambda_{0}\right)(1-\alpha)+64 \cos \left(2 \lambda_{0}\right) \alpha \lambda_{0}}\left[(1+x-x \alpha) \sin \left(2 \lambda_{0}(x-1)\right)\right. \\
& -2 \lambda_{0} \alpha(x+1) \cos \left(2 \lambda_{0}(x-1)\right)+(1-x+x \alpha) \sin \left(2 \lambda_{0}(x+1)\right) \\
& \left.-2 \lambda_{0} \alpha(x-1) \cos \left(2 \lambda_{0}(x+1)\right)\right], \\
C_{5}(x)= & \frac{A B}{16 \sin \left(2 \lambda_{0}\right)(1-\alpha)+32 \alpha \lambda_{0} \cos \left(2 \lambda_{0}\right)}\left[(1+x-x \alpha) \sin \left(2 \lambda_{0}(x-1)\right)\right. \\
& -2 \lambda_{0} \alpha(1+x) \cos \left(2 \lambda_{0}(x-1)\right)+(1-x+x \alpha) \sin \left(2 \lambda_{0}(x+1)\right) \\
& \left.-2 \lambda_{0} \alpha(x-1) \cos \left(2 \lambda_{0}(x+1)\right)\right] .
\end{aligned}
$$


Then we can obtain the full expression for $u_{2}(x, \theta)$ by substituting all the expressions for $C_{i}$ into equation (4.12).

For example, after using the eigenvalue equation given in (4.8), the solution of $C_{3}(x)$ can be simplified as:

$$
C_{3}(x)=-\frac{1}{16 \lambda_{0}}\left(A^{2}+B^{2}\right)\left(\sin \left(2 \lambda_{0} x\right)\right)
$$

The balance at $O\left(\epsilon^{3}\right)$ is of the form

$$
\lambda_{0}^{2} u_{3 \theta \theta}-u_{3 x x}=\lambda_{0}^{2} \nu u_{1 x x \theta}-2 \lambda_{0} \delta u_{1 \theta \theta}-u_{1} u_{2 x}-u_{2} u_{1 x}
$$

and

$$
u_{3}(0, \theta)=\sin (\theta), \quad(1-\alpha) u_{3}(1, \theta)+\alpha u_{3 x}(1, \theta)=0 .
$$

Multiplying equation (4.17) by $\sin \left(\lambda_{0} x\right) \sin (\theta)$ and $\sin \left(\lambda_{0} x\right) \cos (\theta)$ respectively, and integrating both sides for $0 \leq x \leq 1$ and $0 \leq \theta \leq 2 \pi$, then using the boundary condition in equation (4.18), we obtain the following restrictions on $A$ and $B$ :

$$
\begin{array}{r}
\xi_{1} A^{3}+\xi_{2} B^{2}+\xi_{3} \nu B+\xi_{4} \delta A=\pi \lambda_{0}, \\
\xi_{5} B^{3}+\xi_{6} A^{2}+\xi_{7} \nu A+\xi_{8} \delta B=0 .
\end{array}
$$

where $\xi_{j}, 1 \leq j \leq 8$ are functions depending on $\alpha$ and $\lambda_{0}$, given by 


$$
\begin{aligned}
& \xi_{1}=\frac{1}{\zeta}\left(\left(\left(-32 \lambda_{0}^{2}-8\right) \alpha^{3}+\left(25+38 \lambda 0^{2}\right) \alpha^{2}-26 \alpha+9\right) \pi \cos \left(6 \lambda_{0}\right)\right. \\
& +\left(4 \pi \alpha\left(-12-37 \lambda_{0}^{2} \alpha+24 \alpha+16 \lambda_{0}^{2} \alpha^{2}-12 \alpha^{2}\right)\right) \cos \left(4 \lambda_{0}\right) \\
& +\pi\left(9-8 \alpha^{3}-602 \lambda_{0}^{2} \alpha^{2}-32 \lambda_{0}^{2}+608 \lambda_{0}^{2} \alpha^{3}+200 \lambda_{0}^{2} \alpha-26 \alpha-64 \alpha^{2} \lambda_{0}^{4}+25 \alpha^{2}\right) \cos \left(2 \lambda_{0}\right) \\
& \left.+4 \pi \alpha\left(12+12 \alpha^{2}-24 \alpha-87 \lambda_{0}^{2} \alpha+50 \lambda_{0}^{2}+16 \lambda_{0}^{2} \alpha^{2}+16 \alpha \lambda_{0}^{4}\right)\right), \\
& \xi_{2}=\frac{1}{\zeta}\left(-\pi\left(-9+8 \alpha^{3}-25 \alpha^{2}-38 \lambda_{0}^{2} \alpha^{2}+32 \lambda_{0}^{2} \alpha^{3}+26 \alpha\right) \cos \left(6 \lambda_{0}\right)\right. \\
& +4 \pi \alpha\left(-12-37 \lambda_{0}^{2} \alpha+24 \alpha+16 \lambda_{0}^{2} \alpha^{2}-12 \alpha^{2}\right) \cos \left(4 \lambda_{0}\right) \\
& -\pi\left(9-8 \alpha^{3}-602 \lambda_{0}^{2} \alpha^{2}-32 \lambda_{0}^{2}+608 \lambda_{0}^{2} \alpha^{3}+200 \lambda_{0}^{2} \alpha-26 \alpha-64 \alpha^{2} \lambda_{0}^{4}+25 \alpha^{2}\right) \cos \left(2 \lambda_{0}\right) \\
& \left.+4 \pi \alpha\left(12+12 \alpha^{2}-24 \alpha-87 \lambda_{0}^{2} \alpha+50 \lambda_{0}^{2}+16 \lambda_{0}^{2} \alpha^{2}+16 \alpha \lambda_{0}^{4}\right)\right), \\
& \xi_{3}=\frac{1}{\zeta}\left(512 \pi \lambda_{0}^{4}\left(2 \lambda_{0}^{2} \alpha^{2}+\alpha^{2}-2 \alpha+1\right) \cos \left(4 \lambda_{0}\right)+2048 \pi \lambda_{0}^{6} \alpha \cos \left(2 \lambda_{0}\right)\right. \\
& \left.+512 \pi \lambda_{0}^{4}\left(-1-\alpha^{2}+6 \lambda_{0}^{2} \alpha^{2}-4 \lambda_{0}^{2} \alpha+2 \alpha\right)\right), \\
& \xi_{4}=\frac{1}{\zeta}\left(1024 \lambda_{0} \pi\left(2 \lambda_{0}^{2} \alpha^{2}+\alpha^{2}-2 \alpha+1\right) \cos \left(4 \lambda_{0}\right)+4096 \pi \lambda_{0}^{3} \alpha \cos \left(2 \lambda_{0}\right)\right. \\
& \left.+1024 \lambda_{0} \pi\left(-1-\alpha^{2}+6 \lambda_{0}^{2} \alpha^{2}-4 * \lambda_{0}^{2} \alpha+2 \alpha\right)\right), \\
& \xi_{5}=-\pi\left(32 \lambda_{0}^{2} \alpha^{3}-25 \alpha^{2}+8 \alpha^{3}-9+26 \alpha-38 \lambda_{0}^{2} \alpha^{2}\right) \cos \left(6 \lambda_{0}\right) \\
& +4 \pi \alpha\left(-37 \lambda_{0}^{2} \alpha+24 \alpha-12 \alpha^{2}-12+16 \lambda_{0}^{2} \alpha^{2}\right) \cos \left(4 \lambda_{0}\right) \\
& -\pi\left(25 \alpha^{2}+608 \lambda_{0}^{2} \alpha^{3}-26 \alpha-32 \lambda_{0} 0^{2}+9-64 \alpha^{2} \lambda_{0}^{4}+200 \lambda_{0}^{2} \alpha-602 \lambda_{0}^{2} \alpha^{2}-8 \alpha^{3}\right) \cos \left(2 \lambda_{0}\right) \\
& +4 \pi \alpha\left(50 \lambda_{0}^{2}+12 \alpha^{2}+16 \alpha \lambda_{0}^{4}+16 \lambda_{0}^{2} \alpha^{2}-24 \alpha-87 \lambda_{0}^{2} \alpha+12\right), \\
& \xi_{6}=-\pi\left(32 \lambda_{0}^{2} \alpha^{3}-25 \alpha^{2}+8 \alpha^{3}-9+26 \alpha-38 \lambda_{0}^{2} \alpha^{2}\right) \cos \left(6 \lambda_{0}\right) \\
& +4 \pi \alpha\left(-37 \lambda_{0}^{2} \alpha+24 \alpha-12 \alpha^{2}-12+16 \lambda_{0}^{2} \alpha^{2}\right) \cos \left(4 \lambda_{0}\right) \\
& -\pi\left(25 \alpha^{2}+608 \lambda_{0}^{2} \alpha^{3}-26 \alpha-32 \lambda_{0}^{2}+9-64 \alpha^{2} \lambda_{0}^{4}+200 \lambda_{0}^{2} \alpha-602 \lambda_{0}^{2} \alpha^{2}-8 \alpha^{3}\right) \cos \left(2 \lambda_{0}\right) \\
& +4 \pi \alpha\left(50 \lambda_{0}^{2}+12 \alpha^{2}+16 \alpha \lambda_{0}^{4}+16 \lambda_{0}^{2} \alpha^{2}-24 \alpha-87 \lambda_{0}^{2} \alpha\right) \\
& \xi_{7}=-512 \pi \lambda_{0}^{4}\left(-2 \alpha+1+\alpha^{2}+2 \lambda_{0}^{2} \alpha^{2}\right) \cos \left(4 \lambda_{0}\right)-2048 \pi \lambda_{0}^{6} \alpha \cos \left(2 \lambda_{0}\right) \\
& -512 \pi \lambda_{0}^{4}\left(6 \lambda_{0}^{2} \alpha^{2}+2 \alpha-1-4 \lambda_{0}^{2} \alpha-\alpha^{2}\right), \\
& \xi_{8}=1024 \lambda_{0} \pi\left(-2 \alpha+1+\alpha^{2}+2 \lambda_{0}^{2} \alpha^{2}\right) \cos \left(4 \lambda_{0}\right)+4096 \pi \lambda_{0}^{3} \alpha \cos \left(2 \lambda_{0}\right) \\
& +1024 \lambda_{0} \pi\left(6 \lambda_{0}^{2} \alpha^{2}+2 \alpha-1-4 \lambda_{0}^{2} \alpha-\alpha^{2}\right)
\end{aligned}
$$


with $\zeta=4096 \alpha \lambda_{0}^{2}(-1+\alpha)\left(\cos \left(2 \lambda_{0}\right)-1\right)$

Recall that choose the previous form of the second boundary condition in equation (4.2) in order to study different cases of $\alpha$. Consider now the case $\alpha=0.5$. Then the corresponding eigenvalue is obtained by substituting $\alpha=0.5$ in the eigenvalue equation (4.8) and solving for $\lambda_{0}$. We find that $\lambda_{0}=2.02876$. However, the solvability conditions for $A$ and $B$ are given by

$$
\begin{gathered}
0.109918674 A^{3}+0.1099186740 A B^{2}-31.81116883 \nu B-7.619368708 \delta A=\pi \lambda_{0} \\
0.109918674 B^{3}+0.1099186740 B A^{2}+31.81116883 \nu A-7.619368708 \delta B=0 .
\end{gathered}
$$

To find the values of $A$ and $B$, we solve the two equations in (4.21), choosing $\nu=1$ and $\delta=0$. We obtain the following values:

$$
A=0.2779029826 \times 10^{-4}, B=-0.2003551215 .
$$

The dominant first harmonic approximation is completed and can be used to compare to the numerical solution, as we will discuss in the next section.

\subsection{Numerical Results and Comparison with Sin- gle Mode}

In this section, we carry out the numerical solution of the nonlinear wave model problem given by equation (4.1) with boundary condition in (4.2). First, we should note that the numerical solution shown here is chosen for a specific damping coefficient $\nu=1$. We will test this model for a variety values of $\alpha$ to observe its effect. We obtain the following simulation results by choosing the initial conditions: $u_{0}(x)=u_{1}(x, 0)$ and $u_{0 \theta}(x)=\partial u_{1}(x, 0)$. For example, as we are considering the single-mode case, 
then the initial conditions are $u_{0}(x)=B \sin \left(\lambda_{0} x\right)$ and $u_{0 \theta}(x)=A \sin \left(\lambda_{0} x\right)$. Next, we compare the analytic approximate solution from Section 4.2 with the numerical solution. For more details on numerical methods see [21, 26, 37, 40, 55]. We consider three cases corresponding to different values of $\alpha$. In all cases, approximate analytic solutions with one mode for the leading-order will be compared with exact numerical solutions.

\section{Case 1: $\alpha=0.5$}

The numerical method applied involves discretizing the time variable using the RungeKutta method of order four and the centered difference formula to approximate the partial derivative with respect to $x$. We set the time step $\Delta t=0.01$. The result of the simulation is shown in Figure 4.1 below. This figure illustrates the comparison between the numerical and analytic solutions for the case $\alpha=0.5$. The result obtained for the nonlinear damping model problem in equation (4.1) shows that there is good agreement between the leading-order approximation and numerical solutions.

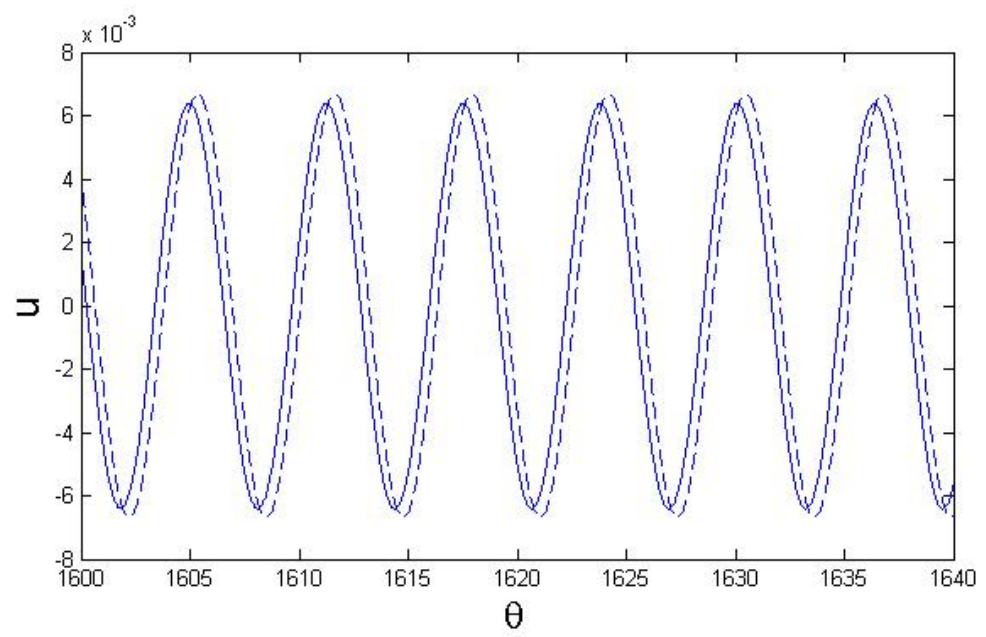

Figure 4.1: Comparison of numerical solution (solid line) to leading-order approximation (dashed line) with $\alpha=0.5$.

Case 2: $\alpha=0.95$

In order to obtain the comparison between the numerical solution and analytic ap- 
proximation, we first need to find the amplitudes at leading-order for the approximate solution for this case.

Considering the form of the leading-order solution consisting of only one mode as the case $\alpha=0.5$, and solving the eigenvalue equation in (4.8) yields $\lambda_{0}=1.60360533$. Then, using the same technique, the Fredholm alternative provides the following condition on the amplitudes $A$ and $B$ :

$$
\begin{aligned}
& -0.1449910724 A B^{2}-0.1449910723 A^{3}-10.59984165 \nu B-5.140873292 \delta A=\pi \lambda_{0} \\
& -0.1449910723 B^{3}-5.140873292 \delta B-0.1449910724 B A^{2}+10.59984165 \nu A=0 .
\end{aligned}
$$

Solving these equations with $\nu=1$ and $\delta=0$, the amplitudes $A$ and $B$ are:

$$
A=-0.146851217 \times 10^{-2}, B=-0.4752737630 .
$$

Figure 4.2 compares the continuous numerical solution for case $\alpha=0.95$ with the leading-order perturbation solution $u_{1}(x, \theta)$ using one mode. It is clear from this figure, that when the value of $\alpha$ is close to $\alpha=1$, then we do not obtain as nice a comparison between the exact numerical solution and one-mode leading-order approximation. In fact, the comparison in the case $\alpha=0.5$ is noticeably better than the case $\alpha=0.95$ as shown in Figure 4.1. 


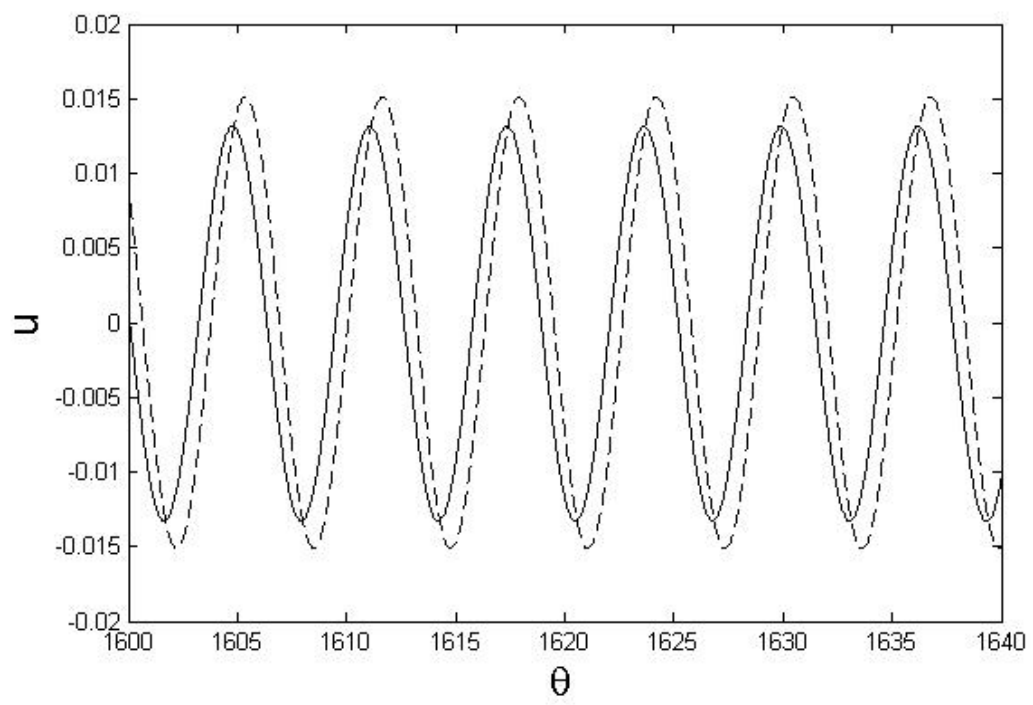

Figure 4.2: Comparison of numerical solution (solid line) to single-mode leadingorder approximation (dashed line) with $\alpha=0.95$.

Case 3: $\alpha=1$

For this case, the boundary conditions at (4.1) become

$$
u_{1}(0, \theta)=0, \quad u_{1 x}(1, \theta)=0
$$

In fact, the analysis for this case is similar to the case $\alpha=0.5$, but it is easier due to the simple boundary conditions that are given in (4.25). We aim to obtain a leadingorder approximate solution, which is based on determining the two amplitudes $A$ and $B$. Then we will compare the continuous numerical solution with the leading-order approximate solution.

We start by studying the analytic solution as in the previous case. Notice that the boundary conditions for the eigenfunctions $\phi(x)$ are: $\phi(0)=0$ and $\phi^{\prime}(1)=0$, yielding an eigenvalue equation $\lambda_{0} \cos \left(\lambda_{0}\right)=0$. Thus the eigenvalues are commensurate and given by $\lambda_{n}=n \frac{\pi}{2}$, where $n$ is an odd integer of the form $n=2 k-1$, where $k \geq 1$. From Chapter 3, a continuous solution is expected. 
Applying the Fredholm alternative theorem yields the following restriction for the amplitudes $A$ and $B$ :

$$
\begin{aligned}
& -0.1656699251 A^{3}-9.563115158 \nu B-4.934802205 \delta A-0.1656699251 A B^{2}=\pi \lambda_{0} \\
& -0.1656699251 B^{3}-0.1656699251 B A^{2}+9.563115158 \nu A-4.934802205 \delta B=0 .
\end{aligned}
$$

Solving these equations with $\nu=1$ and $\delta=0$, we obtain the amplitudes $A$ and $B$ :

$$
A=-0.2380328522 \times 10^{-2}, B=-0.5160135703 .
$$

Next, using the same numerical scheme, and starting with the initial conditions $u_{0}(x)=B \sin \left(\lambda_{0} x\right)$ and $u_{0 \theta}(x)=A \sin \left(\lambda_{0} x\right)$, the numerical solution is computed. Finally, we compare the approximate analytic solution with the numerical solution. In Figure 4.3, we compare the numerical continuous solution where the damping coefficient is $\nu=1$ with the leading-order analytic solution where the amplitudes are given by equation (4.27).

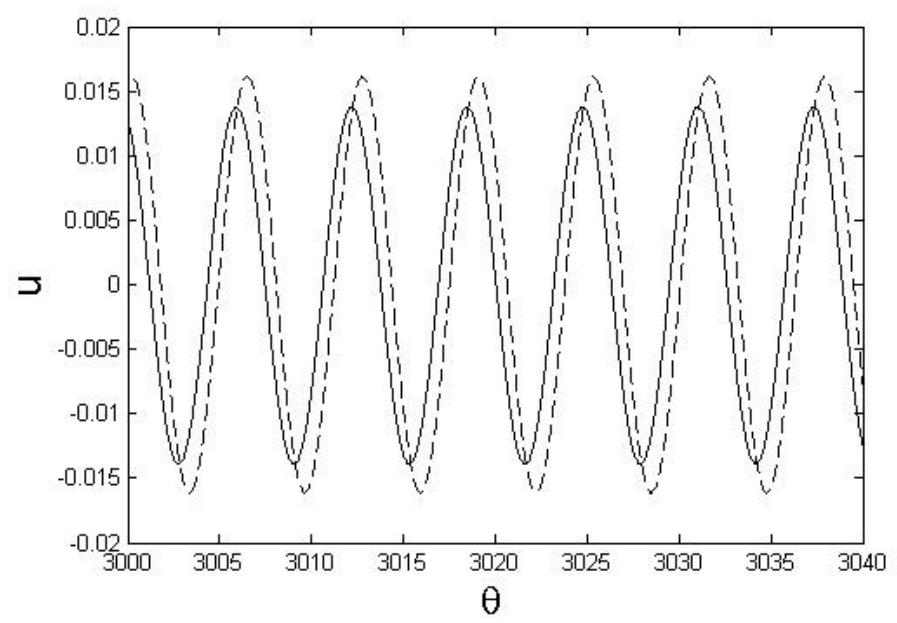

Figure 4.3: Comparison of numerical solution (solid line) to leading-order approximation (dashed line) with $\alpha=1$.

Notice that, from Figures 4.2 and 4.3, we do not see as nice agreement between 
numerical and analytical approximation for both cases when $\alpha=1$ and $\alpha=0.95$

The validity of the analytic approximation given in Section 4.2 depends on the incommensurate nature of the underlying linear eigenvalue. So these analyses are not correct. For example, if $3 \lambda$ is an eigenvalue, then we will obtain more secular terms at this order and the analysis breaks down. However, to achieve good agreement between the numerical solution and the analytic approximation, higher modes may be added to the leading-order at this point. In the next section, we will study analytic solutions with higher modes and explain how to obtain the solvability conditions.

\subsection{Weakly Nonlinear Analysis with Higher Modes}

In some cases, as noted above, the comparison between the analytic solution with one mode and the numerical solution do not provide good agreement. So we need to add higher modes at the leading-order.

Consider the leading-order, including arbitrary modes, as given by

$$
u_{1}(x, \theta)=\sum_{k=1}^{\infty} \phi_{2 k-1}(x)\left[A_{2 k-1} \sin ((2 k-1) \theta)+B_{2 k-1} \cos ((2 k-1) \theta)\right]
$$

with the boundary condition given in equation (4.6).

As we discussed for the single mode case, following the same analytic approach, we obtain the eigenfunction $\phi_{2 k-1}(x)$ satisfying the boundary-value problem

$$
\left((2 k-1) \lambda_{0}\right)^{2} \phi_{2 k-1}(x)+\phi_{2 k-1}^{\prime \prime}(x)=0,
$$

with $\phi_{2 k-1}(0)=0$ and $(1-\alpha) \phi_{2 k-1}(1)+\alpha \phi_{2 k-1}^{\prime}(1)=0$. 
Therefore the eigenfunction is

$$
\phi_{2 k-1}(x)=\sin \left((2 k-1) \lambda_{0} x\right)
$$

Upon applying the boundary condition on $\phi_{2 k-1}(x), \lambda_{0}$ is determined by the eigenvalue equation:

$$
(\alpha-1) \sin \left((2 k-1) \lambda_{0} x\right)=\alpha(2 k-1) \lambda_{0} \cos \left((2 k-1) \lambda_{0} x\right)
$$

Using the leading-order solution $u_{1}(x, \theta)$ given by (4.28) and (4.30) the inhomogeneous part of equation (4.10), we find that the form of $u_{2}(x, \theta)$ could be given by the following :

$$
u_{2}(x, \theta)=B(x)+\sum_{j=2,4,6}^{\infty}\left[C_{j}(x) \sin (j \theta)+C_{j+1}(x) \cos (j \theta)\right]
$$

where $B(x), C_{j}(x)$ and $C_{j+1}(x)$ can be determined subject to the boundary conditions

$$
\begin{aligned}
& B(0)=0,(\alpha-1) B(1)=\alpha B^{\prime}(1), \\
& C_{j}(0)=0,(\alpha-1) C_{j}(1)=\alpha C_{j}^{\prime}(1) .
\end{aligned}
$$

We should notice that the solution of all $B(x), C_{j}(x)$ functions exist since $j \lambda_{0}$ are not assumed to be eigenvalues. In fact, all even multiplies of the fundamental frequency are not resonant at this point.

Similarly, by continuing to apply the same procedure, we can find the form of the solution at the third order at $O\left(\epsilon^{3}\right)$ and higher orders.

As we explained above, we study now in more detail how we can obtain the analytic solutions where the leading-order approximate solution contains two modes. For this case, the form of leading-order solution subject to (4.28) will be similar to the one 
when there was a single mode but with additional terms.

In fact, the form of the leading-order solution with two modes is given by

$$
u_{1}(x, \theta)=\phi_{1}(x)[A \sin (\theta)+B \cos (\theta)]+\phi_{3}(x)[C \sin (3 \theta)+D \cos (3 \theta)]
$$

where $A, B, C$ and $D$ are arbitrary amplitudes at this order.

As we discussed in the case of one mode, by replacing $\phi_{1}(x)$ by $\phi(x)$, we see that it satisfies the same eigenvalue equation (4.8). Likewise, $\phi_{3}(x)$ satisfies the eigenvalue problem:

$$
9 \lambda_{0}^{2} \phi_{3}(x)+\phi_{3}^{\prime \prime}(x)=0, \phi_{3}(0)=0, \text { and }(1-\alpha) \phi_{3}(1)+\alpha \phi_{3}^{\prime}(1)=0 .
$$

Provided $3 \lambda_{0}$ is not an eigenvalue, then $\phi_{3}(x)$ exists and the classical solution is

$$
\phi_{3}(x)=C_{3} \cos \left(3 \lambda_{0} x\right)+C_{4} \sin \left(3 \lambda_{0} x\right)
$$

Therefore, applying the boundary condition for $\phi_{3}(x)$ in (4.35), the fundamental frequency $\lambda_{0}$ is determined by the eigenvalue equation:

$$
(\alpha-1) \sin \left(3 \lambda_{0}\right)=\alpha\left(3 \lambda_{0}\right) \cos \left(3 \lambda_{0}\right)
$$

Using the boundary conditions of $u_{1}(x, \theta)$ in (4.6), the leading-order approximation for this case is given by

$$
\begin{aligned}
& u_{1}(x, \theta)=\frac{1}{2} A\left[\cos \left(\lambda_{0} x-\theta\right)-\cos \left(\lambda_{0} x+\theta\right)\right]+\frac{1}{2} B\left[\sin \left(\lambda_{0} x-\theta\right)+\sin \left(\lambda_{0} x+\theta\right)\right] \\
& +\frac{1}{2} C\left[\cos \left(3 \lambda_{0} x-3 \theta\right)-\cos \left(3 \lambda_{0} x+3 \theta\right)\right]+\frac{1}{2} D\left[\sin \left(3 \lambda_{0} x-3 \theta\right)+\sin \left(3 \lambda_{0} x+3 \theta\right)\right] .
\end{aligned}
$$




\section{Solvability conditions of amplitudes $A, B, C$, and $D$}

In the first correction to linear theory in the perturbation solution to (4.1) and (4.2) (i.e., at $\left.O\left(\epsilon^{2}\right)\right) u_{2}(x, \theta)$ satisfies the same equation and the boundary conditions in (4.10) and (4.11) respectively. However, after substituting the two-mode leadingorder solution given in (4.38) into the right-hand side of equation (4.10), it should be noted that the form of $u_{2}(x, \theta)$ subject to (4.32) is given by

$$
\begin{aligned}
u_{2}(x, \theta)=c_{3}(x) & +c_{4}(x) \cos (2 \theta)+c_{5}(x) \sin (2 \theta)+c_{6}(x) \cos (4 \theta)+c_{7}(x) \sin (4 \theta) \\
& +c_{8}(x) \cos (6 \theta)+c_{9}(x) \sin (6 \theta) .
\end{aligned}
$$

In order to find the full expression of $u_{2}(x, \theta)$, we substitute the form of $u_{2}$ in $(4.39)$ into the second-order equation given by (4.10) then group all terms of trigonometric and non-trigonometric functions. After some manipulation, we obtain that the equations for $c_{i}(x)$ in $u_{2}$, where $3 \leq i \leq 9$, satisfy the following forms:

$$
\begin{aligned}
& c_{3}^{\prime \prime}(x)-\frac{\lambda_{0}}{4}\left(B^{2}+A^{2}\right) \sin \left(2 \lambda_{0} x\right)-\frac{3 \lambda_{0}}{4}\left(C^{2}+D^{2}\right) \sin \left(6 \lambda_{0} x\right)=0 \\
& c_{4}^{\prime \prime}(x)+4 \lambda_{0}^{2} c_{4}(x)=(B D+A C) \lambda_{0} \sin \left(4 \lambda_{0} x\right)-\left(2 B D+2 A C+A^{2}-B^{2}\right) \frac{\lambda_{0}}{4} \sin \left(2 \lambda_{0} x\right), \\
& c_{5}^{\prime \prime}(x)+4 \lambda_{0}^{2} c_{5}(x)=(B C-A D) \lambda_{0} \sin \left(4 \lambda_{0} x\right)-(B C-A D-A B) \frac{\lambda_{0}}{2} \sin \left(2 \lambda_{0} x\right), \\
& c_{6}^{\prime \prime}(x)+16 \lambda_{0}^{2} c_{6}(x)=(B D-A C) \lambda_{0} \sin \left(4 \lambda_{0} x\right)+(A C-B D) \frac{\lambda_{0}}{2} \sin \left(2 \lambda_{0} x\right), \\
& c_{7}^{\prime \prime}(x)+16 \lambda_{0}^{2} c_{7}(x)=(A D+B C) \lambda_{0} \sin \left(4 \lambda_{0} x\right)-(A D+B C) \frac{\lambda_{0}}{2} \sin \left(2 \lambda_{0} x\right), \\
& c_{8}^{\prime \prime}(x)+36 \lambda_{0}^{2} c_{8}(x)=\frac{3 \lambda_{0}}{4}\left(D^{2}-C^{2}\right) \sin \left(6 \lambda_{0} x\right), \\
& c_{9}^{\prime \prime}(x)+36 \lambda_{0}^{2} c_{9}(x)=\frac{3 \lambda_{0}}{2} D C \sin \left(6 \lambda_{0} x\right),
\end{aligned}
$$


with the boundary conditions

$$
c_{i}(0)=0, \quad(1-\alpha) c_{i}(1)+\alpha c_{i}^{\prime}(1)=0 i=3, \ldots 9 .
$$

Now, we can determine each function $c_{i}(x)$ at $u_{2}(x, \theta)$. The solution of $c_{3}(x)$ is given by

$$
\begin{aligned}
c_{3}(x)= & \frac{1}{48 \lambda_{0}}\left(3 x(1-x \alpha)\left(A^{2}+B^{2}\right) \sin \left(2 \lambda_{0}\right)+6 x \alpha \lambda_{0}\left(A^{2}+B^{2}\right) \cos \left(2 \lambda_{0}\right)\right. \\
& +x(1-\alpha)\left(C^{2}+D^{2}\right) \sin \left(6 \lambda_{0}\right)+6 x \alpha \lambda_{0}\left(C^{2}+D^{2}\right) \cos \left(6 \lambda_{0}\right)-3\left(A^{2}+B^{2}\right) \sin \left(2 \lambda_{0} x\right) \\
& \left.-\left(C^{2}+D^{2}\right) \sin \left(6 \lambda_{0} x\right)\right) .
\end{aligned}
$$

The solution of $c_{4}(x)$ can be obtained by solving the $c_{4}$ equation in (4.40) subject to the boundary conditions $c_{4}(0)=0,(\alpha-1) c_{4}(1)-\alpha c_{4}^{\prime}(1)=0$. We get

$$
\begin{aligned}
c_{4}(x) & =\frac{1}{96 \lambda_{0} \sin \left(2 \lambda_{0}\right)(\alpha-1)-192 \alpha \lambda_{0}^{2} \cos \left(2 \lambda_{0}\right)}\left(N_{1} \sin \left(2 \lambda_{0}(x-1)\right)+N_{2} \cos \left(2 \lambda_{0}(x-1)\right)\right. \\
& +N_{3} \sin \left(2 \lambda_{0}(x+1)\right)+N_{4} \cos \left(2 \lambda_{0}(x+1)\right)+N_{5} \sin \left(2 \lambda_{0}(x-2)\right)+N_{6} \cos \left(2 \lambda_{0}(x-2)\right) \\
& +N_{7} \sin \left(2 \lambda_{0}(2 x-1)\right)+N_{8} \cos \left(2 \lambda_{0}(2 x-1)\right)+N_{9} \sin \left(2 \lambda_{0}(2 x+1)\right)+N_{10} \cos \left(2 \lambda_{0}(2 x+1)\right) \\
& \left.+N_{11} \sin \left(2 \lambda_{0}(x+2)\right)+N_{12} \cos \left(2 \lambda_{0}(x+2)\right)\right)
\end{aligned}
$$

where the coefficients of the trigonometric functions $N_{i}, 1 \leq i \leq 12$ depend on the 
value of $\alpha$ and the amplitudes $A, B, C$ and $D$. The formula for all $N_{i}$ are given by

$$
\begin{aligned}
& N_{1}=(1+x-x \alpha)\left(3 A^{2}-3 B^{2}+6 B D+6 A C\right) \lambda_{0} \\
& N_{2}=(1+x)\left(6 B^{2}-6 A^{2}-12 A C-12 D B\right) \alpha \lambda_{0}^{2} \\
& N_{3}=(1-x+x \alpha)\left(3 A^{2}-3 B^{2}+6 B D+6 A C\right) \lambda_{0} \\
& N_{4}=(x-1)\left(6 B^{2}-6 A^{2}-12 A C-12 D B\right) \alpha \lambda_{0}^{2} \\
& N_{5}=-(D B-A C) 16 \alpha \lambda_{0} \\
& N_{6}=4(\alpha-1)(D B+A C), \\
& N_{7}=8 \alpha \lambda_{0}(D B+A C) \\
& N_{8}=4(1-\alpha)(A C+D B), \\
& N_{9}=8 \alpha \lambda_{0}(D B+A C), \\
& N_{10}=4(\alpha-1)(A C+D B), \\
& N_{11}=-16 \alpha \lambda_{0}(D B+A C), \\
& N_{12}=4(1-\alpha)(A C+D B),
\end{aligned}
$$

Similarly, the solution of $c_{5}(x)$ is given by

$$
\begin{aligned}
c_{5}(x) & =\frac{1}{48 \lambda_{0} \sin \left(2 \lambda_{0}\right)(\alpha-1)-96 \alpha \lambda_{0}^{2} \cos \left(2 \lambda_{0}\right)}\left(M_{1} \sin \left(2 \lambda_{0}(x-1)\right)+M_{2} \cos \left(2 \lambda_{0}(x-1)\right)\right. \\
& +M_{3} \sin \left(2 \lambda_{0}(x+1)\right)+M_{4} \cos \left(2 \lambda_{0}(x+1)\right)+M_{5} \sin \left(2 \lambda_{0}(x-2)\right)+M_{6} \cos \left(2 \lambda_{0}(x-2)\right) \\
& +M_{7} \sin \left(2 \lambda_{0}(2 x-1)\right)+M_{8} \cos \left(2 \lambda_{0}(2 x-1)\right)+M_{9} \sin \left(2 \lambda_{0}(2 x+1)\right)+M_{10} \cos \left(2 \lambda_{0}(2 x+1)\right) \\
& \left.+M_{11} \sin \left(2 \lambda_{0}(x+2)\right)+M_{12} \cos \left(2 \lambda_{0}(x+2)\right)\right)
\end{aligned}
$$

where $M_{i}, 1 \leq i \leq 12$ depend on $\alpha$ and the amplitudes $A, B, C$ and $D$ and are given by: 


$$
\begin{aligned}
& M_{1}=3 \lambda_{0}(x \alpha-x-1)(A D+A B-B C), \\
& M_{2}=6 \alpha \lambda_{0}^{2}(1+x)(A B+A D-B C), \\
& M_{3}=3 \lambda_{0}(x \alpha-x+1)(B C-A B-A D), \\
& M_{4}=6 \alpha \lambda_{0}^{2}(x-1)(A B+A D-B C), \\
& M_{5}=8 \alpha \lambda_{0}(A D-B C), \\
& M_{6}=2(\alpha-1)(B C-A D), \\
& M_{7}=4 \alpha \lambda_{0}(B C-A D), \\
& M_{8}=2(1-\alpha)(B C-A D), \\
& M_{9}=4 \alpha \lambda_{0}(B C-A D), \\
& M_{10}=2(\alpha-1)(B C-A D), \\
& M_{11}=-8 \alpha \lambda_{0}(B C-A D), \\
& M_{12}=2(\alpha-1)(A D-B C),
\end{aligned}
$$

Similarly, by solving the remaining equations in (4.40) with corresponding boundary conditions (4.41), then analogous expressions for $c_{i}(x), 6 \leq i \leq 9$, are obtained. Then we can obtain the full expression for $u_{2}(x, \theta)$ by substituting the $c_{i}$ expressions into equation (4.39).

The balance equation at $O\left(\epsilon^{3}\right)$ in the perturbation expansion is the same one given by (4.17), with the corresponding boundary conditions (4.18).

At first glance, finding the solvability equations for the amplitudes $A, B, C$ and $D$, where $u_{1}(x, \theta)$ is given in (4.38), appears to be amenable to similar analysis as the case of a single mode. However, there is a substantial difference. Using Maple, this presents some additional challenges due to the large expression for $u_{2}(x, \theta)$. In fact, this is clear when applying the Fredholm alternative theorem to the right-hand side of equation (4.17). So, to handle this problem, we break up the integral and use Riemann sums to approximate the second definite integral. 
To obtain the solvability equations, we start by multiplying both sides of equation (4.17) by $\sin \left(\lambda_{0} x\right) \sin (\theta), \sin \left(\lambda_{0} x\right) \cos (\theta), \sin \left(3 \lambda_{0} x\right) \sin (3 \theta)$ and $\sin \left(3 \lambda_{0} x\right) \cos (3 \theta)$ respectively. Then, integrating first with respect $\theta$ from 0 to $2 \pi$ and with respect to $x$ from 0 to 1 . However, to reduce the complexity of the expressions for $u_{2}(x, \theta)$, we will approximate the result of the definite integral with respect to $x$ by applying Riemann sums.

\subsection{Numerical Simulation and Comparison for Two Modes}

Recall that in Section 4.3, we carried out the numerical solution and compared with the analytic solution contains only a single-mode. In this part, we compare the numerical solution with two modes at the leading-order, and then we will show how adding more modes to the leading-order plays an important role in improving the comparison results.

Notice that, from Figure 4.2, we do not see good agreement between the numerical solution and the analytical approximations. So we study now case where there are two modes at the leading-order. Applying the two-mode analytical approach as explained at the end of Section 4.4, we multiply equation (4.17) by $\sin \left(\lambda_{0} x\right) \sin (\theta)$, $\sin \left(\lambda_{0} x\right) \cos (\theta), \sin \left(3 \lambda_{0} x\right) \sin (3 \theta)$ and $\sin \left(3 \lambda_{0} x\right) \cos (3 \theta)$. Then the solvability conditions are obtained by integrating over the domain $\theta \in(0,2 \pi)$ and then using Riemann sums to approximate the definite integral over the domain $x \in(0,1)$. Thus, putting conditions on the resonant amplitudes $A, B, C$ and $D$, the coefficient terms of the resulting equations depend on the solutions determined at order $O(\epsilon)$ and $O\left(\epsilon^{2}\right)$ and on the value of the variable $\alpha$ at the boundary condition, along with the other equation parameters such as $\nu$ and $\delta$. 
Case 1: $\alpha=0.95$

Consider now the case where $\alpha=0.95$. Following the weakly analytic approach as discussed in Section 4.4 with $\nu=1$ and $\delta=0$ (on resonance), we obtain following solvability conditions:

$$
\begin{aligned}
& -0.14394 A^{3}-0.18377 B^{2} C+0.18692 A C^{2}+0.18377 A^{2} C+0.36754 A B D+0.18692 A D^{2}- \\
& 0.14394 A B^{2}-11.63564 B=\pi \lambda_{0}, \\
& 0.18692 B C^{2}+0.18692 B D^{2}-0.14394 A^{2} B-0.18377 B^{2} D+0.18377 A^{2} D-0.3675 A B C- \\
& 0.14394 B^{3}+11.6356 A=0, \\
& 0.00179 A^{3}-0.16997 B^{2} C-0.16997 A^{2} C-0.11395 C^{3}+0.005383 A B^{2}-0.11395 C D^{2}- \\
& 313.4914 D=0, \\
& -0.00538 A^{2} B-0.11395 D^{3}-0.16997 B^{2} D-0.16997 A^{2} D+0.00179 B^{3}-0.11395 C^{2} D+ \\
& 313.4914330 C=0 .
\end{aligned}
$$

Solving these equations for the amplitudes $A, B, C$, and $D$, we obtain the following values:

$$
A=-0.001004, B=-0.43297, C=4.6467 \times 10^{-7}, D=-3.279 \times 10^{-9} .
$$

By comparing the values of amplitudes are given by (4.48) with the corresponding values in Section 4.3, where $\alpha=0.95$ and the leading-order contained only one mode, we observe that the values of amplitudes for $A$ and $B$ are changed. However, the values of $C$ and $D$ are small. This difference accounts for the detailed impact of the system of equations given by (4.47) on the values of $A$ and $B$.

The comparison between the numerical solution and the leading-order approximation 
using one and two modes is shown in Figure 4.4. We notice that adding higher modes improves the comparison results. There is now better quantitative agreement.

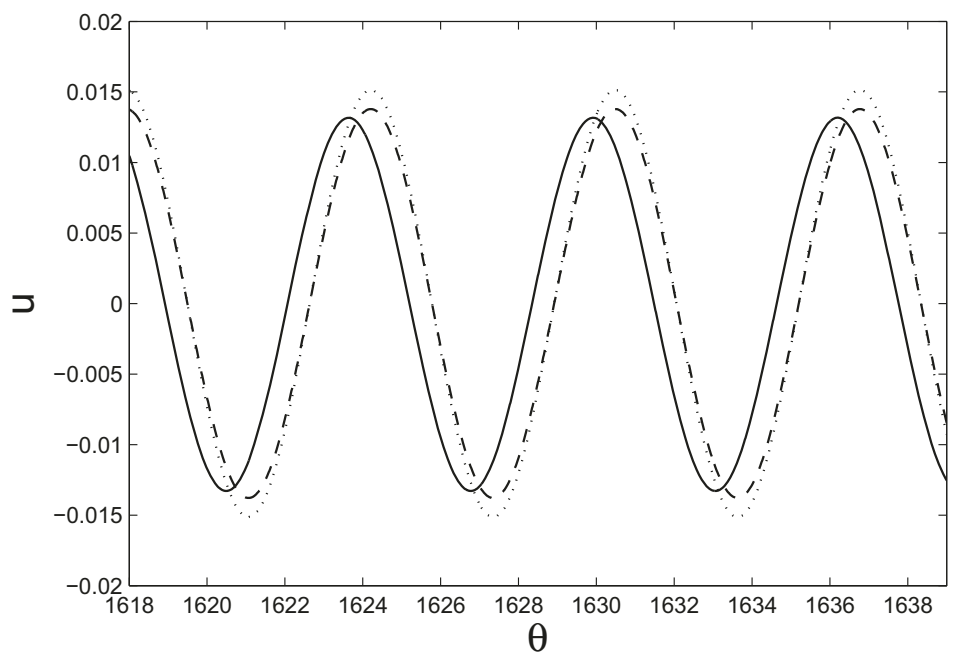

Figure 4.4: Comparison of the two-mode leading-order approximation solution (dashed line) and numerical solution (solid line) and single-mode (dotted line) with $\alpha=0.95$.

Case 2: $\alpha=1$

Consider now the case $\alpha=1$ where the form of the leading-order approximation contains two modes as given by equation (4.38). The analytical solution can be obtained by using the same method to find the analytical solution with higher modes for the case $\alpha=0.95$.

After applying the analytical approximation solution for higher modes to the case $\alpha=1$ as we discussed in the previous section, we get the system of cubic equations for the amplitude-frequency relations given by 


$$
\begin{aligned}
& -0.16567 A^{3}-0.1943 C B^{2}-0.16567 A B^{2}+0.1902 A D^{2}+0.1943 C A^{2}+0.1902 A C^{2}+ \\
& 0.3886 B D A-9.56312 \nu B-4.9348 \delta A=\pi \lambda_{0} \\
& -0.16567 B A^{2}+0.19021 B C^{2}+0.19021 B D^{2}-0.1943 D B^{2}+0.1943 D A^{2}-0.16567 B^{3}- \\
& 4.9348 \delta B+9.56312 \nu A-0.38861 A B C=0 \\
& -0.00614 A^{3}-0.16567 C^{3}-0.2393 C B^{2}+0.018408 A B^{2}-0.2393 C A^{2}-44.413 \delta C- \\
& 258.204 \nu D-0.16567 C D^{2}=0 \\
& -0.01841 B A^{2}-0.16567 D^{3}-44.413 \delta D+258.204 \nu C-0.2393 D B^{2}-0.2393 D A^{2}+ \\
& 0.00614 B^{3}-0.16567 D C^{2}=0 .
\end{aligned}
$$

Solving these equations with $\nu=1$ and $\delta=0$ for the amplitudes $A, B, C$ and $D$, we obtain the following values :

$$
A=-0.001628 B=-0.469108, C=0.26023 \times 10^{-5}, D=-2.75781 \times 10^{-8} .
$$

It should be noted that the values of the amplitudes $A$ and $B$ given in (4.50) are different from the equivalent values found in Section 4.3, where there is a single mode in the leading-order approximate solution with $\alpha=1$.

Figure 4.5 shows the comparison between the numerical solution and the leadingorder approximate solution using two-modes and a single- mode, respectively. It is clear now using two-modes obtains a better agreement than using only a single mode. 


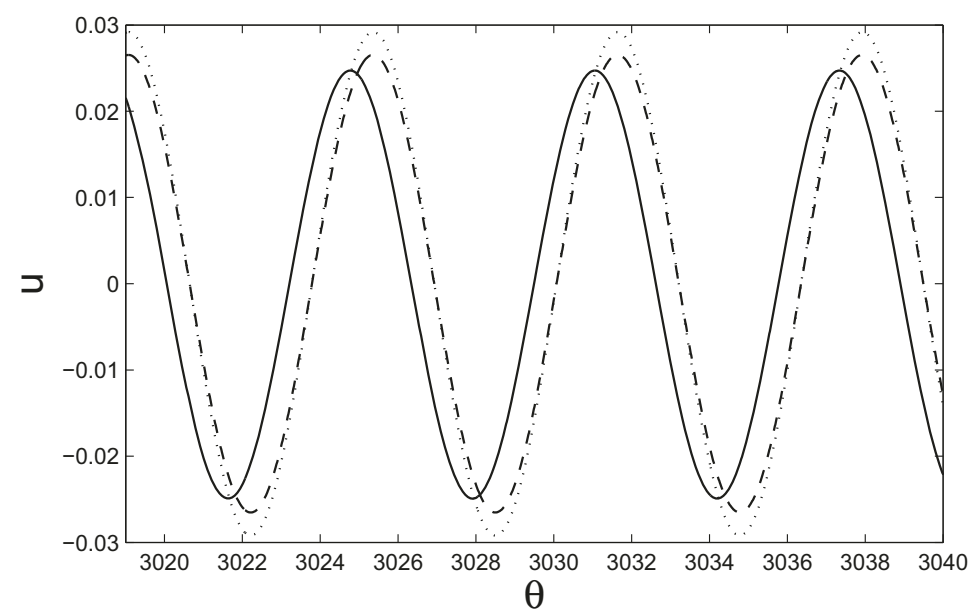

Figure 4.5: Comparison of numerical solution (solid line) to two-modes leading-order approximation (dashed line) and one-mode (dotted line) with $\alpha=1$.

\section{Role of Higher modes: Two modes}

We notice from Figures 4.4 and 4.5 how the second mode plays an important role in improving the agreement between the numerical solution and the analytical approximation solution. Here we study in more detail the role of higher modes as $\alpha$ varies. We will explain how we should add higher modes when the value of $\alpha$ is close to $\alpha=1$. However, we will also show that, when the values of $\alpha$ are far away from 1 , we do not need to add higher modes.

Figure 4.6 illustrates the comparison of single-mode leading-order approximation for the case $\alpha=0.9999$ and numerical solution, but in Figure 4.7 we add more modes and see how it plays a primary role to obtain an excellent result for the comparison. These two figures show that it is important to add higher modes when $\alpha$ is close to 1 . In fact, we study the case $\alpha=0.9999$ in order to show whether we need to add more modes as $\alpha \rightarrow 1$. This we will discuss in more detail in Section 4.9, where a detailed process for determination of the truncation of modes will be obtained. 


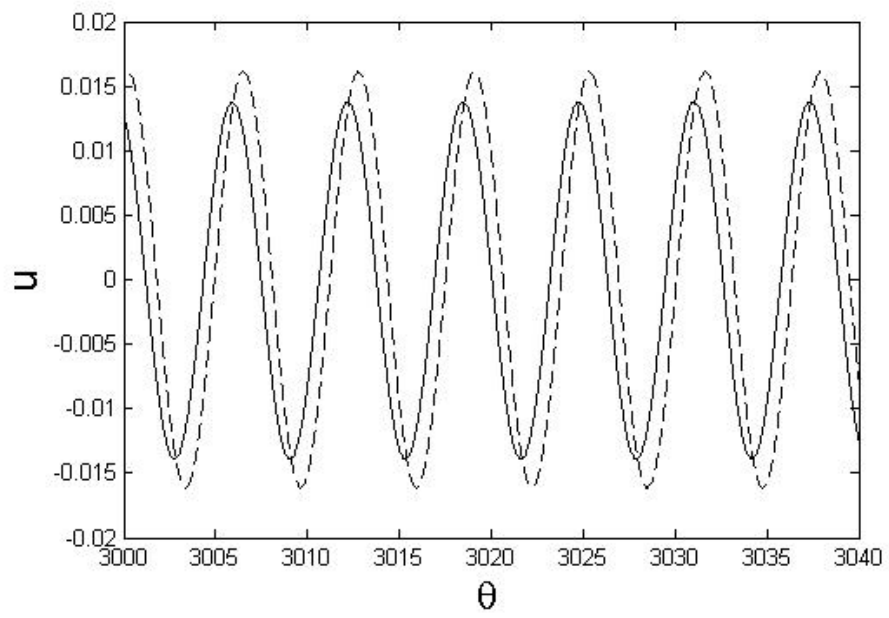

Figure 4.6: Comparison of numerical solution (solid line) to leading-order approximation with one mode (dashed line) with $\alpha=0.9999$.

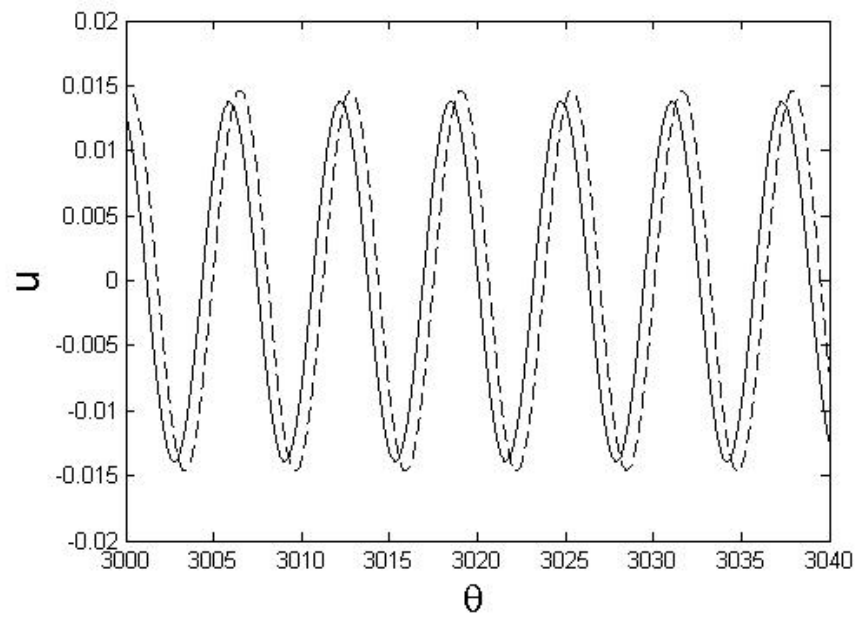

Figure 4.7: Comparison of numerical solution (solid line) to leading-order approximation with two-modes (dashed line) with $\alpha=0.9999$. 
The next two figures show that, when the value of $\alpha$ is far away from one, we do not need to add more modes. Figure 4.8 shows the comparison results between a single-mode approximate solution and the numerical solution for the case $\alpha=0.6$. However, Figure 4.9 illustrates the comparison for same $\alpha$ but using two modes in the leading-order approximation solution. It is clear that there is almost no difference between single mode and higher modes when the value of $\alpha$ is far away from one.

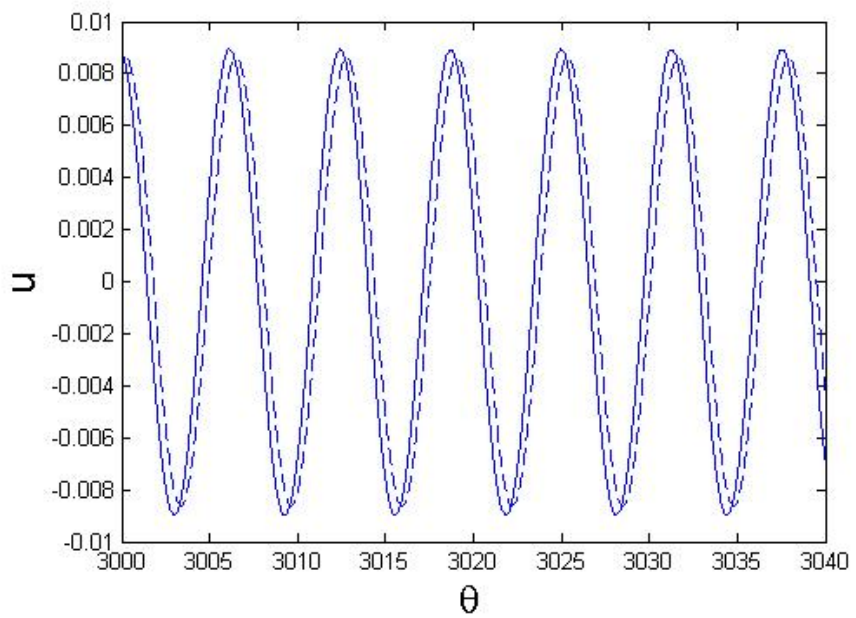

Figure 4.8: Comparison of numerical solution (solid line) to leading-order approximation with one mode (dashed line) with $\alpha=0.6$.

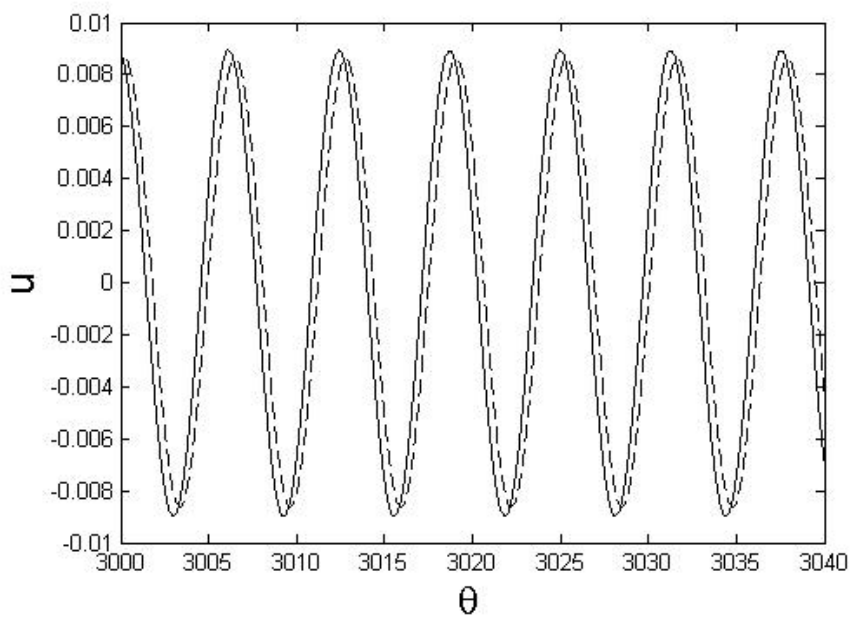

Figure 4.9: Comparison of numerical solution (solid line) to leading-order approximation with two-modes (dashed line) with $\alpha=0.6$. 


\subsection{Comparison of Amplitude}

The comparison between the numerical solution and leading-order approximation was obtained in the previous section. In this part, we focus in more detail on the amplitude comparison as parameter vary. This plays a main role in explaining a natural transition between using the single-mode solution and the higher-modes solution.

To obtain this comparison, we need to find the amplitude from the numerical solution and the others from the analytical approximation solution when using single and multiple modes.

For the analytic amplitude; i.e., $A \_A$ we need the two values for amplitudes $A$ and $B$ that were found when we discussed the analytical solution. However, for the amplitude from the numerical solution, we will use the fast Fourier transform (FFT) function.

Figure 4.10 shows the comparison between amplitudes within this regime based on numerical simulation on resonance $\delta=0$ and under variation of $\alpha$. We notice that this comparison not only shows a good quantitative agreement between the three amplitude curves but this approximation also captures the qualitative of the amplitude variation within the transition regime. Figure 4.10 shows the absolute value of the amplitude obtained by numerical simulation, and analytically where the leadingorder contains a single mode $A_{-} A[1]$ or two-mode $A_{-} A[2]$. Also, this figure illustrates how the two-mode amplitude curve (dashed line) is closer to the numerical curve more than the one-mode curve, which explains how important it is to include the higher modes.

Figure 4.11 shows the response (amplitude-frequency) curve, $A-\delta$. In order to generate the amplitude-frequency plot, we consider the case $\alpha=1$. In this plot, we compare three amplitude-frequency curves. The first one is when using only a single mode at the leading-order. To obtain this curve, we vary the detuning frequency 
$\delta$, and the amplitude-frequency relation arises from the cubic equations (4.26) for $A$ and $B$. The second curve is for two modes, and this can be obtained by changing $\delta$ in the system of equations given by (4.49) for amplitudes $A, B, C$ and $D$. The last curve is for the amplitude-frequency obtained numerically.

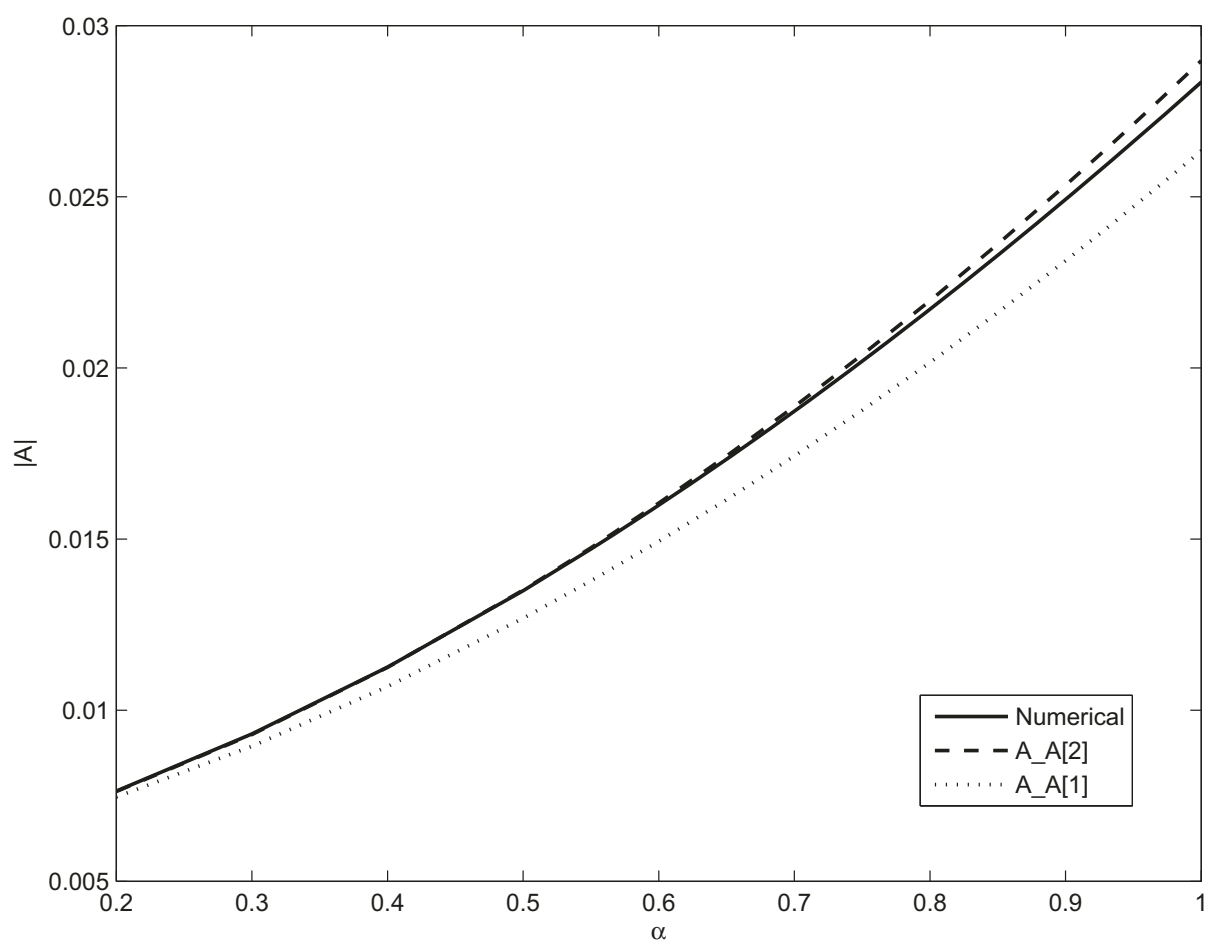

Figure 4.10: Comparison of amplitude curves from numerical solution (solid line) and the leading-order approximation using two modes (dashed line) and using single mode (dotted line). 


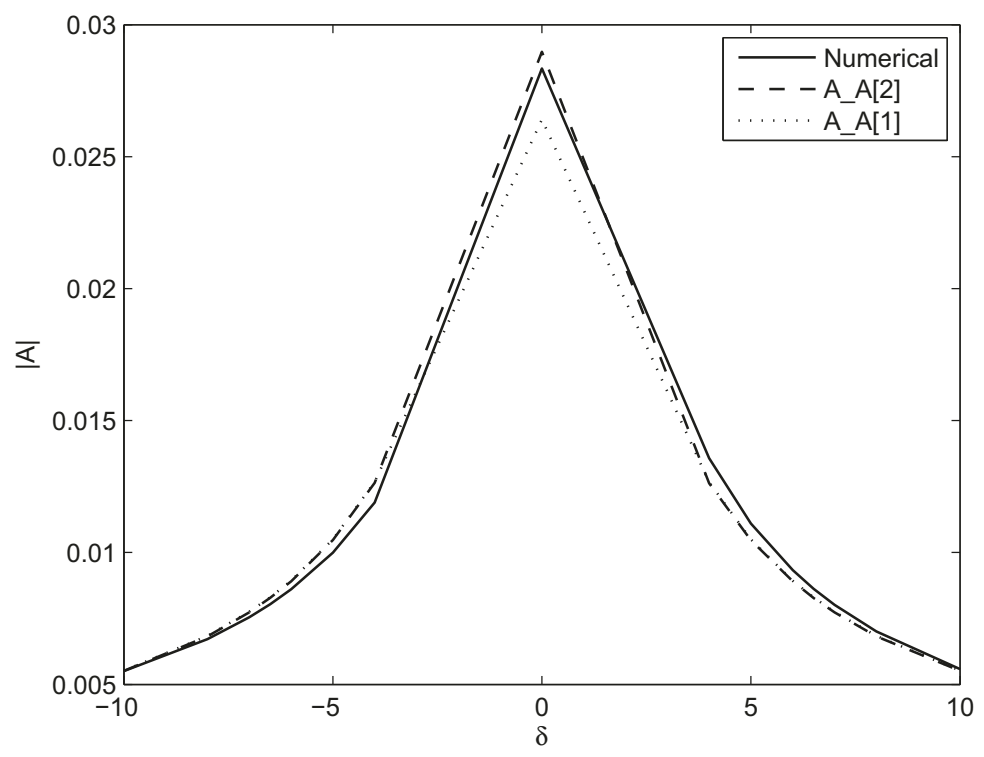

Figure 4.11: The amplitude-frequency curves from numerical solution (solid line) and the leading-order approximation with two modes (dashed line) and single mode (dotted line) when $\alpha=1$. 


\subsection{Stability Analysis and the Effect of Damping}

In this section, we will use the multiple-scale method to study the analytic solution for the case where the nonlinear model problem has a secular term. Then, in the second part, we determine the fixed points for the new system after adding a slow time and study their stability. We start by rescaling the problem and considering a valid approximation for the solution.

Let us consider the nonlinear model problem given in equation (4.1) with the boundary conditions (4.2).

We notice, for some cases of $\alpha$ ( for example when $\alpha=1$ ) in the analytical solution of this problem as discussed in Section 4.2, there will arise at order $O\left(\epsilon^{3}\right)$ a term that grows linearly in $t$, a secular term. Then, to remove this term, we need to add a slow time and use the multiple-scale method as explained in more detail in Chapter 2.

Notice that, in order to eliminate the secular term, we need the slow time to arise at the third order. To get that, we introduce a new variable $\tau=\epsilon^{2} \theta$. Multiple-scale analysis provides a solution as a function of both variables $\theta$ and $\tau$, considering them as independent variables but with $\frac{\partial \tau}{\partial \theta}=\epsilon^{2}$.

Consider the following perturbation expansions

$$
\begin{aligned}
U(x ; \theta) & =\epsilon U_{1}(x ; \theta, \tau)+\epsilon^{2} U_{2}(x ; \theta, \tau)+\epsilon^{3} U_{3}(x ; \theta, \tau)+O\left(\epsilon^{4}\right), \\
\lambda & =\lambda_{0}+\epsilon^{2} \delta+\epsilon^{3} \lambda_{3}+\cdots .
\end{aligned}
$$

The second derivative is

$$
\frac{d^{2} U_{i}}{d t^{2}}=\frac{\partial^{2} U_{i}}{\partial \theta^{2}}+2 \epsilon^{2} \frac{\partial U_{i}}{\partial \tau \partial \theta}+\epsilon^{4} \frac{\partial^{2} U_{i}}{\partial \tau^{2}}
$$


We substitute the expansions (4.51) and (4.52) into the nonlinear model problem in equation (4.1) and equating terms of $O(\epsilon)$ and $O\left(\epsilon^{2}\right)$.

At $O(\epsilon)$, we find that $U_{1}(x ; \theta, \tau)$ satisfies the equation

$$
\lambda_{0}^{2} U_{1 \theta \theta}-U_{1 x x}=0
$$

and

$$
U_{1}(0, \theta, \tau)=0,(1-\alpha) U_{1}(1, \theta, \tau)+\alpha U_{1 x}(1, \theta, \tau)=0
$$

We consider the leading-order having the same form as before (as in Section 4.1), but the main difference now is the amplitudes are functions of $\tau$; i.e. $U_{1}(x, \theta, \tau)=$ $\phi(x)(A(\tau) \sin (\theta)+B(\tau) \cos (\theta))$. Substituting $U_{1}$ in equation (4.54), we find that $\phi(x)$ satisfies the same eigenvalue problem given in equation (3.11), and the eigenvalue $\lambda_{0}$ can be determined by the eigenvalue equation :

$$
(1-\alpha) \sin \left(\lambda_{0}\right)+\alpha \lambda_{0} \cos \left(\lambda_{0}\right)=0
$$

Therefore, the leading-order solution is given by

$$
U_{1}(x, \theta, \tau)=\sin \left(\lambda_{0} x\right)[A(\tau) \sin (\theta)+B(\tau) \cos (\theta)]
$$

At $O\left(\epsilon^{2}\right)$, we find that $U_{2}(x ; \theta, \tau)$ satisfies the equation

$$
\lambda_{0}^{2} U_{2 \theta \theta}-U_{2 x x}+U_{1} U_{1 x}=0
$$

and

$$
U_{2}(0, \theta, \tau)=0,(1-\alpha) U_{2}(1, \theta, \tau)+\alpha U_{2 x}(1, \theta, \tau)=0
$$

After substituting the leading-order solution $U_{1}(x ; \theta, \tau)$ given by $(4.57)$ into equation 
(4.58), we determine that the form of $U_{2}$ should be

$U_{2}(x, \theta, \tau)=\left(A^{2}(\tau)+B^{2}(\tau)\right) C_{3}(x)-\left(A^{2}(\tau)-B^{2}(\tau)\right) C_{4}(x) \cos (2 \theta)+A(\tau) B(\tau) C_{5}(x) \sin (2 \theta)$

Substituting this form into equation (4.58), we obtain that $C_{3}(x), C_{4}(x)$ and $C_{5}(x)$ are determined by the following equations :

$$
\begin{aligned}
-\left(A^{2}(\tau)+B^{2}(\tau)\right) C_{3}^{\prime \prime}(x)-\frac{\lambda_{0}}{4}\left(A^{2}(\tau)+B^{2}(\tau)\right) \sin \left(2 \lambda_{0} x\right) & =0 \\
-\left(B^{2}(\tau)-A^{2}(\tau)\right) C_{4}^{\prime \prime}(x)-4 \lambda_{0}^{2} C_{4}(x)\left(B^{2}(\tau)-A^{2}(\tau)\right) & =-\frac{\lambda_{0}}{4} \sin \left(2 \lambda_{0} x\right)\left(B^{2}(\tau)-A^{2}(\tau)\right) \\
B(\tau) A(\tau) C_{5}^{\prime \prime}(x)+4 \lambda_{0}^{2} B(\tau) A(\tau) C_{5}(x) & =\frac{\lambda_{0}}{2} A B \sin \left(2 \lambda_{0} x\right) .
\end{aligned}
$$

Then we can find the whole expression of $U_{2}(x, \theta, \tau)$ by solving these equations with the corresponding boundary conditions

$$
C_{i}(0)=0,(1-\alpha) C_{i}(1)+\alpha C_{i}^{\prime}(1)=0, i=3,4,5
$$

The balance at $O\left(\epsilon^{3}\right)$ is of the form

$$
\lambda_{0}^{2} U_{3 \theta \theta}-U_{3 x x}=\lambda_{0}^{2} \nu U_{1 x x \theta}-2 \lambda_{0} \delta U_{1 \theta \theta}-\left(U_{1} U_{2 x}+U_{2} U_{x}\right)-2 \lambda_{0}^{2} U_{1 \tau \theta}
$$

and

$$
U_{3}(0, \theta, \tau)=\sin (\theta),(1-\alpha) U_{3}(1, \theta, \tau)+\alpha U_{3 x}(1, \theta, \tau)=0
$$

Multiplying equation (4.63) by $\sin \left(\lambda_{0} x\right) \sin (\theta)$ and $\sin \left(\lambda_{0} x\right) \cos (\theta)$ respectively, and integrating both sides for $0 \leq x \leq 1,0 \leq \theta \leq 2 \pi$, then using the boundary condition 
in equation (4.64), we obtain restrictions on $A(\tau)$ and $B(\tau)$ given by the system:

$$
\left(\begin{array}{l}
\frac{\partial A(\tau)}{\partial \tau} \\
\frac{\partial B(\tau)}{\partial \tau}
\end{array}\right)=\frac{1}{P\left(\lambda_{0}, \alpha\right)}\left(\begin{array}{l}
F_{1}\left(A(\tau), B(\tau), \lambda_{0}, \alpha\right) \\
F_{2}\left(A(\tau), B(\tau), \lambda_{0}, \alpha\right)
\end{array}\right)
$$

where the function $P$ depends on $\lambda_{0}$ and $\alpha$, but $F_{1}$ and $F_{2}$ depend on $A(\tau), B(\tau), \lambda_{0}$ and $\alpha$. The formulas for the functions $P, F_{1}$ and $F_{2}$ are given by

$$
\begin{aligned}
& P=1024 \lambda_{0}^{2}\left(\left(1+\alpha^{2}+2 \lambda_{0}^{2} \alpha^{2}-2 \alpha\right) \cos \left(4 \lambda_{0}\right)+4 \lambda_{0}^{2} \alpha \cos \left(2 \lambda_{0}\right)+6 \lambda_{0}^{2} \alpha^{2}-4 \lambda_{0}^{2} \alpha-1+2 \alpha-\alpha^{2}\right), \\
& F_{1}=\kappa_{4}\left(B(\tau) A^{2}(\tau)+B^{3}(\tau)\right)+\kappa_{5} A(\tau) \\
& F_{2}=\kappa_{1}\left(A^{3}(\tau)+B^{2}(\tau) A(\tau)\right)+\kappa_{2} B(\tau)+\kappa_{3},
\end{aligned}
$$

where the coefficients $\kappa_{i}, 1 \leq i \leq 5$ depend on the values of $\alpha$ and $\lambda_{0}$. The formulas for all $\kappa_{i}$ are given by : 


$$
\begin{aligned}
\kappa_{1} & =\left[8 \alpha^{3}\left(1+4 \lambda_{0}^{2}\right)-\alpha^{2}\left(25-38 \lambda_{0}^{2}\right)+26 \alpha-9\right] \cos \left(6 \lambda_{0}\right) \\
& +\left[\alpha^{3}\left(48-64 \lambda_{0}^{2}\right)+\alpha^{2}\left(148 \lambda_{0}^{2}-96\right)+48 \alpha\right] \cos \left(4 \lambda_{0}\right) \\
& +\left[\alpha^{3}\left(608 \lambda_{0}^{2}-8\right)-\alpha^{2}\left(64 \lambda_{0}^{4}+602 \lambda_{0}^{2}-25\right)+\alpha\left(200 \lambda_{0}^{2}-26\right)-32 \lambda_{0}^{2}+9\right] \cos \left(2 \lambda_{0}\right) \\
& -\alpha^{3}\left(48+64 \lambda_{0}^{2}\right)+\alpha^{2}\left(348 \lambda_{0}^{2}-64 \lambda_{0}^{4}+96\right)-\alpha\left(48+200 \lambda_{0}^{2}\right), \\
\kappa_{2}= & {\left[512 \lambda_{0}^{4}\left(2 \alpha-1-\alpha^{2}\left(2 \lambda_{0}^{2}+1\right)\right)\right] \cos \left(4 \lambda_{0}\right)-2048 \lambda_{0}^{6} \alpha \cos \left(2 \lambda_{0}\right)+\alpha^{2} \lambda_{0}^{4}\left(512-3072 \lambda_{0}^{2}\right) } \\
& +1024 \alpha \lambda_{0}^{4}\left(2 \lambda_{0}^{2}-1\right)+512 \lambda_{0}^{2}, \\
\kappa_{3}= & 4096 \lambda 0^{3} \alpha(1-\alpha)\left(1-\cos \left(2 \lambda_{0}\right)\right), \\
\kappa_{4}= & \left(8 \alpha^{3}\left(1+4 \lambda_{0}^{2}\right)+\alpha^{2}\left(25-38 \lambda_{0}^{2}\right)+26 \alpha-9\right) \cos \left(6 \lambda_{0}\right) \\
& +\left[\left(-64 \lambda 0^{2}+48\right) \alpha^{3}+\left(148 \lambda 0^{2}-96\right) \alpha^{2}+48 \alpha\right] \cos \left(4 \lambda_{0}\right) \\
& +\left[\left(-8+608 \lambda 0^{2}\right) \alpha^{3}+\left(-64 \lambda 0^{4}-602 \lambda 0^{2}+25\right) \alpha^{2}+\left(-26+200 \lambda 0^{2}\right) \alpha+9-32 \lambda 0^{2}\right] \cos \left(2 \lambda_{0}\right) \\
& +\left(-64 \lambda 0^{2}-48\right) \alpha^{3}+\left(-64 \lambda 0^{4}+96+348 \lambda 0^{2}\right) \alpha^{2}+\left(-48-200 \lambda 0^{2}\right) \alpha, \\
\kappa_{5} & =\left[512 \lambda 0^{4}\left(\alpha^{2}\left(2 \lambda 0^{2}+1\right)-2 \alpha+1\right] \cos \left(4 \lambda_{0}\right)+2048 \lambda 0^{6} \alpha \cos (2 \lambda 0)\right. \\
& -512 \lambda 0^{4}\left(\alpha^{2}\left(6 \lambda 0^{2}-1\right)+\alpha\left(2-4 \lambda 0^{2}\right)-1\right) .
\end{aligned}
$$

Before studying the stability of this system, recall that the hyperbolic point of the system $X^{\prime}=F(X)$ is a fixed point $X^{*}$ of the system $\left(F\left(X^{*}\right)=0\right)$ where none of the eigenvalues of the Jacobian matrix $D F\left(X^{*}\right)$ has zero real part.

The stability of the hyperbolic fixed point $X^{*}$ is determined by the sign of the real part of the eigenvalues of Jacobian matrix. If the eigenvalues of the Jacobian all have negative real part, then $X^{*}$ is asymptotically stable. If at least one eigenvalue of $D F\left(X^{*}\right)$ has positive real part, then $X^{*}$ is an unstable fixed point.

The Jacobian matrix of the system in (4.65) is given by 


$$
D F\left(X^{*}\right)=\left(\begin{array}{cc}
2 \kappa_{4} B(\tau) A(\tau)+\kappa_{5} & \kappa_{4}\left(3 B^{2}(\tau)+A^{2}(\tau)\right) \\
\kappa_{1}\left(3 A^{2}(\tau)+B^{2}(\tau)\right) & \kappa_{1}(2 A(\tau) B(\tau))+\kappa_{2}
\end{array}\right) .
$$

Consider now the case when $\alpha=1$. The eigenvalue equation becomes $\tan \left(\lambda_{0}\right)=0$, where the fundamental eigenvalue is $\lambda_{0}=\frac{\pi}{2}$. Following the analogous approach as we discuss above, we obtain the following restriction on $A(\tau)$ and $B(\tau)$ :

$$
\begin{aligned}
& -9.563115 \nu B(\tau)-0.16567 A(\tau) B^{2}(\tau)-7.75157 \frac{\partial B(\tau)}{\partial \tau}-0.16567 A^{3}(\tau)-4.934 \delta A(\tau)=\frac{\pi^{2}}{2}, \\
& -0.16567 B(\tau) A^{2}(\tau)+9.563115 \nu A(\tau)+7.75157 \frac{\partial A(\tau)}{\partial \tau}-0.16567 B^{3}(\tau)-4.934 \delta B(\tau)=0 .
\end{aligned}
$$

We can rewrite the solvability equations using $\delta=0$ and $\nu=1$ as the system

$$
\left(\begin{array}{l}
\frac{\partial A(\tau)}{\partial \tau} \\
\frac{\partial B(\tau)}{\partial \tau}
\end{array}\right)=\left(\begin{array}{c}
0.02137 B^{3}(\tau)+0.02137 B(\tau) A^{2}(\tau)-1.2337 A(\tau) \\
-0.02137 A^{3}(\tau)-0.02137 A(\tau) B^{2}(\tau)-1.2337 B(\tau)-0.0645 \pi^{2}
\end{array}\right)
$$

The Jacobian matrix of this system is given by

$$
D F\left(X^{*}\right)=\left(\begin{array}{cc}
0.04274 B(\tau) A(\tau)-1.2337 & 0.06411 B^{2}(\tau)+0.02137 A^{2}(\tau) \\
-0.06411 A^{2}(\tau)-0.02137 B^{2}(\tau) & -0.04274 A(\tau) B(\tau)-1.2337
\end{array}\right)
$$

The fixed points of the system in (4.71) are at $A^{*}=-0.0023797$ and $B^{*}=-0.515989$. Then the Jacobian matrix becomes

$$
D F\left(A^{*}, B^{*}\right)=\left(\begin{array}{cc}
-1.23365 & -0.017069 \\
0.00569 & -1.23375
\end{array}\right)
$$


The eigenvalues of this matrix are given by

$$
\begin{aligned}
& \lambda_{1}=-1.2337+0.00985495 i \\
& \lambda_{2}=-1.2337-0.00985495 i
\end{aligned}
$$

Both eigenvalues have negative real part, and this shows that the solution is stable.

To demonstrate the stability for general $\nu$, we study the Jacobian matrix given in (4.71) but varying the parameter $\nu$, then following the same approach as we discussed before to determine the eigenvalues. A summary of the real parts of the eigenvalues and the performance of different $\nu$ 's when $\alpha=1$ is presented in Table 4.1. It is clear that, when $\nu>0$, we obtain a stable solution. But if $\nu<0$, then the solution becomes unstable. Also, it should be noted that, while the values of $\nu$ change to negative, the real parts of eigenvalues change to negative as well.

\begin{tabular}{|c|c|}
\hline$\nu$ & $\Re(\lambda)$ \\
\hline-1 & 1.2337 \\
\hline-0.75 & 0.925275 \\
\hline-0.5 & 0.61685 \\
\hline-0.25 & 0.308428 \\
\hline 0.25 & -0.308428 \\
\hline 0.5 & -0.61685 \\
\hline 0.75 & -0.925275 \\
\hline 1 & -1.2337 \\
\hline
\end{tabular}

Table 4.1: Summary of real eigenvalues of the Jacobian matrix in (4.71).

We thus conclude that, while the details of the analytic solution may vary with changing $\nu$, that does not affect the qualitative nature of the response. In particular, we observe that, from Table 4.1, the variation of $\nu>0$ the solution remains stable.

In the next section, we will discuss the analytic and numerical solution for the same nonlinear model problem in Section 4.1, where the fixed boundary condition at $x=1$ corresponds to the case when $\alpha=0$. However, the forcing amplitude at $x=0$ must be rescaled, as we will discuss in detail. 


\subsection{The Case $\alpha=0$}

In the previous sections, we obtained the analytical solution for the cases when $\alpha=1$ and $0<\alpha<1$. However, in order to obtain good agreement between the exact numerical solution and the leading-order approximation solution, we found that, when the value of $\alpha$ is close to 1 , then the higher modes should be included at the leading-order.

In this section, we consider the case $\alpha=0$ and will explain how the forcing of the nonlinear model problem will affect the perturbation expansion of the analytic solution. Then we compare the analytical approximation solution with the numerical simulation.

\subsubsection{Formulation and linear theory}

Consider the damped nonlinear model problem

$$
\lambda^{2} u_{\theta \theta}-u_{x x}+u u_{x}-\lambda^{2} \mu u_{x x \theta}=0
$$

where $\mu$ is the damping parameter, which is chosen to scale with forcing amplitude as $\mu=m^{\frac{1}{2}} \nu$ with constant $\nu=O(1)$. Over the domain $x \in[0,1]$.

Let $\alpha=0$. Then the boundary conditions in equation (4.6) now become

$$
u(0, \theta)=m \sin (\theta), \quad u(1, \theta)=0,
$$

where $m=\epsilon^{2}$ is the forcing amplitude.

We seek periodic solutions $u(x, \theta)$ in $\theta$, that have the same period as the external 
forcing:

$$
u(x, \theta)=u(x, \theta+2 \pi),
$$

and assume a perturbation expansion of the form:

$$
\begin{aligned}
u(x, \theta) & =m^{\frac{1}{2}} u_{1}(x, \theta)+m u_{2}(x, \theta)+m^{\frac{3}{2}} u_{3}(x, \theta)+\cdots, \\
\lambda & =\lambda_{0}+m^{\frac{1}{2}} \delta+\cdots,
\end{aligned}
$$

where $\left|u_{i}\right|=O(1), i=1,2,3, \ldots, \lambda_{0}$ corresponds to the fundamental resonant frequency and $m^{\frac{1}{2}} \delta$ is the detuning. Therefore, at this point, we need to assume that $m \ll 1$ to ensure the validity of this analysis. Notice that the output is $O\left(m^{\frac{1}{2}}\right)$, while the input is $O(m)$.

Then, at $O\left(m^{\frac{1}{2}}\right)$, we obtain the linear problem:

$$
\lambda_{0}^{2} u_{1 \theta \theta}-u_{1 x x}=0
$$

with

$$
u_{1}(0, \theta)=0, u_{1}(1, \theta)=0 .
$$

We will assume in the leading-order response that the solution $u_{1}(x, \theta)$ of $(4.79)$ and (4.80) is of the form

$$
u_{1}(x, \theta)=\phi(x)(A \sin (\theta)+B \cos (\theta))
$$

where $A$ and $B$ are arbitrary amplitudes and $\phi(x)$ is the eigenfunction corresponding to the fundamental eigenvalue $\lambda_{0}$. 
After substituting $u_{1}(x, \theta)$ into equation (4.79), we see that $\phi(x)$ satisfies the eigenvalue problem:

$$
\lambda_{0}^{2} \phi+\phi^{\prime \prime}=0, \phi(0)=0 \text {, and } \phi(1)=0 .
$$

According to the classical solution of $\phi(x)$ that is given in equation (3.12), it follows that the eigenvalue equation is

$$
\sin \left(\lambda_{0}\right)=0
$$

Notice that the fundamental resonant frequency $\lambda_{0}=\pi$ with commensurate higher mode $\lambda_{n}=n \pi=n \lambda_{0}$, for $n \geq 2$.

Therefore, the leading-order solution is given by

$$
u_{1}(x, \theta)=\sin \left(\lambda_{0} x\right)[A \sin (\theta)+B \cos (\theta)]
$$

\subsubsection{Nonlinear Theory: Amplitude-frequency Relation}

In order to capture the nonlinear effect where the amplitudes $A$ and $B$ will be connected to the frequency through the solvability conditions, we need to continue to the next order.

The equation to determine $u_{2}(x, \theta)$ (i.e., at order $\left.O(m)\right)$ now becomes

$$
\lambda_{0}^{2} u_{2 \theta \theta}-u_{2 x x}=\lambda_{0}^{2} \nu u_{1 x x \theta}-2 \lambda_{0} \delta u_{1 \theta \theta}-u_{1} u_{1 x}
$$

and

$$
u_{2}(0, \theta)=\sin (\theta), u_{2}(1, \theta)=0 \text {. }
$$

At this order, since the commensurate nature of the spectrum, and in fact, $2 \lambda_{0}$ is itself a resonant frequency as well as $2 \theta$-mode now is resonant. So, the perturbation 
expansion that was given in equations (4.3) and (4.4) for the cases when $0<\alpha<1$ and $\alpha=1$ does not work now. However, this explains why we pick up the form of the perturbation expansion given in equations (4.77) and (4.78). However, these reasons together guide us to let the solvability conditions coming to the second order.

Multiplying equation (4.85) by $\sin \left(\lambda_{0} x\right) \sin (\theta)$ and $\sin \left(\lambda_{0} x\right) \cos (\theta)$ respectively, and integrating both sides for $0 \leq x \leq 1,0 \leq \theta \leq 2 \pi$, then using the boundary condition in equation (4.86) with the periodic conditions given in (4.76), we obtain the required amplitude-frequency relation that implies $A$ and $B$ satisfy the following equations:

$$
\begin{aligned}
-\frac{1}{2} B \pi^{3} \lambda_{0}^{2} \nu-A \pi \delta \lambda_{0} & =\pi \lambda_{0} \\
\frac{1}{2} A \pi^{3} \lambda_{0}^{2} \nu-B \pi \delta \lambda_{0} & =0
\end{aligned}
$$

This is a simple system of linear equations in A and B, which can be solved explicitly to obtain

$$
A=-\frac{4 \delta}{4 \delta^{2}+\lambda_{0}^{2} \pi^{4} \nu^{2}}, B=-\frac{2 \pi^{2} \nu \lambda_{0}}{4 \delta^{2}+\lambda_{0}^{2} \pi^{4} \nu^{2}}
$$

Choosing $\nu=1$ and $\delta=0$, and substituting the fundamental eigenvalue $\lambda_{0}=\pi$, we get $A=0$ and $B=-0.06450306884$. With these values of amplitudes, the leadingorder solution given by equation (4.84) is complete.

\subsubsection{Numerical solutions and comparison results}

In this part, we present results of the numerical simulation for solving the nonlinear damping model problem given by equation (4.74) with the boundary conditions in (4.75). Then we compare the numerical solution with the continuous solution obtained by the perturbation methods given in (4.84).

In Figure 4.12, we compare the leading-order approximation solution for $\delta=0$ in- 
cludes a single mode with the exact numerical solution of the equations (4.74) and (4.75), and it shows there is a good agreement. By comparing with the result of the case $\alpha=0$ in Chapter 3 where the response is unbounded, we conclude that the forced boundary and damped coefficient play a main role in the nature of outcome responses.

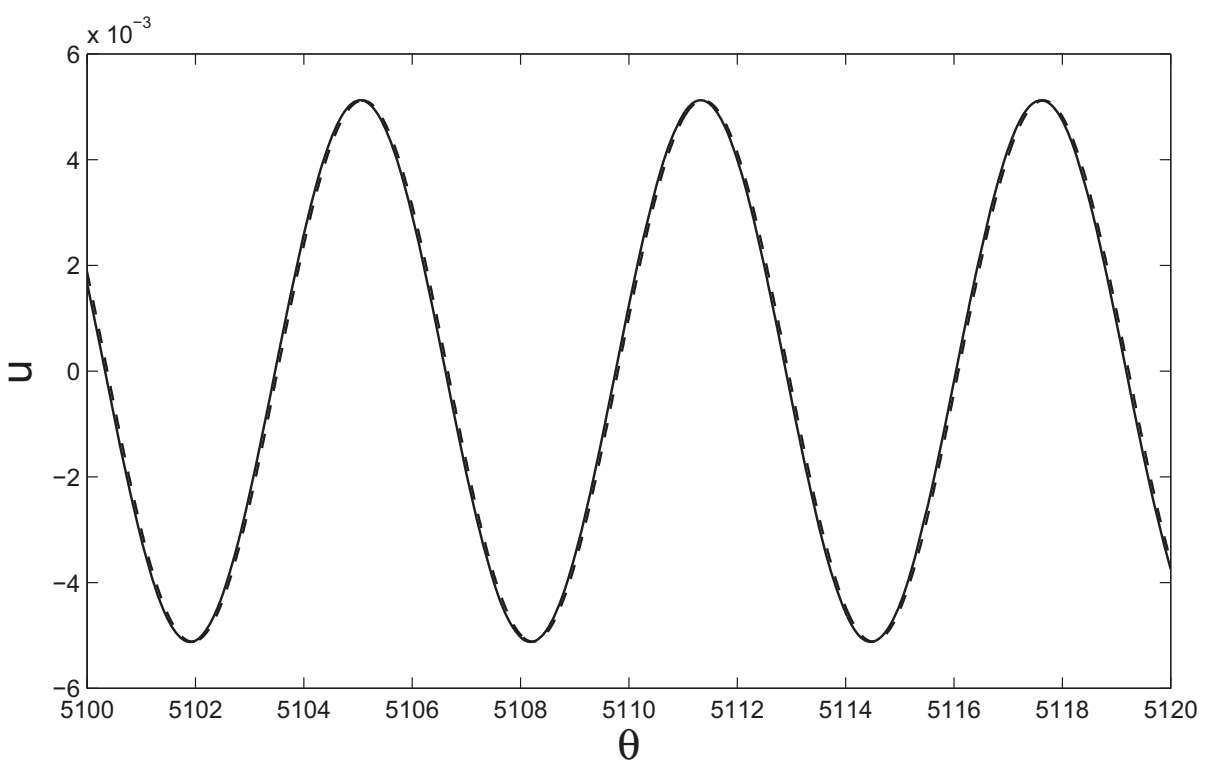

Figure 4.12: Comparison of one-mode approximation solution (dashed line) with $\delta=0$ to numerical solution (solid line) at resonance.

\subsection{Truncation of Modes $N(\alpha)$}

We carried out the weakly nonlinear analysis with higher modes in Section 4.4, and we showed that, for some cases when $\alpha$ is close to $\alpha=1$, the higher modes should be included to improve the comparison between the exact numerical solution and the leading-order approximate solution.

A natural question that arises is how we bridge the gap between using the higher modes that required when $\alpha$ close to $\alpha=1$ and the single-mode expansion for $\alpha$ at or near $\alpha=0$. 
The truncation level that needs to be considered will vary with $\alpha$. In fact, this as a results of the higher modes are not integer multiples of the fundamental. Amundsen et al. [16] carried out the truncation for the geometric parameter $k$. They showed that there is a layer of width $O\left(\epsilon^{2}\right)$, which explains how the geometric variation plays a sufficient role in the truncation with the higher modes.

We consider the case where the arbitrary boundary constant matches with the nonlinear effects, $\alpha=O(\epsilon)$. So, the truncation depends only on the linear eigenvalue equation, but if the order of damping coefficient is different then it could affect in the truncation.

Next, we define the truncation at this point following Amundsen et al. as

$$
\Delta_{n}(\alpha)=\lambda_{n}-n \lambda_{0}, \quad n \geq 2
$$

Then a reasonable truncation points $n$ correspond to $\Delta_{n}(\alpha)=c \epsilon$, where $c$ is an arbitrary constant of order $O(1)$.

In order to study the truncation $N(\alpha)$, we follow the weakly nonlinear analysis with higher mode as in Section 4.4. We note that, as $\alpha \rightarrow 1$, then $N \rightarrow \infty$, and as $\alpha \rightarrow 0$, then $N \rightarrow 1$. So, for given $\alpha$, the leading-order becomes

$$
u_{1}(x, \theta)=\sum_{k=1}^{2 n-1} \phi_{2 k-1}(x)\left[A_{2 k-1} \sin ((2 k-1) \theta)+B_{2 k-1} \cos ((2 k-1) \theta)\right]
$$

where the eigenfunctions $\phi_{2 k-1}(x)$ are given by (4.29), and the corresponding eigenvalue satisfies (4.31).

Figure 4.12 shows how the truncation values $N$ vary with $\alpha$. It is clear from this figure that, when $\alpha$ is close to $\alpha=1$, the number of modes increases $N \rightarrow \infty$. This provides a good explanation why the higher modes must be included in the nonlinear analysis. 
However, for $\alpha$ close to $\alpha=0$, we only need the single mode for the leading-order approximate solution.

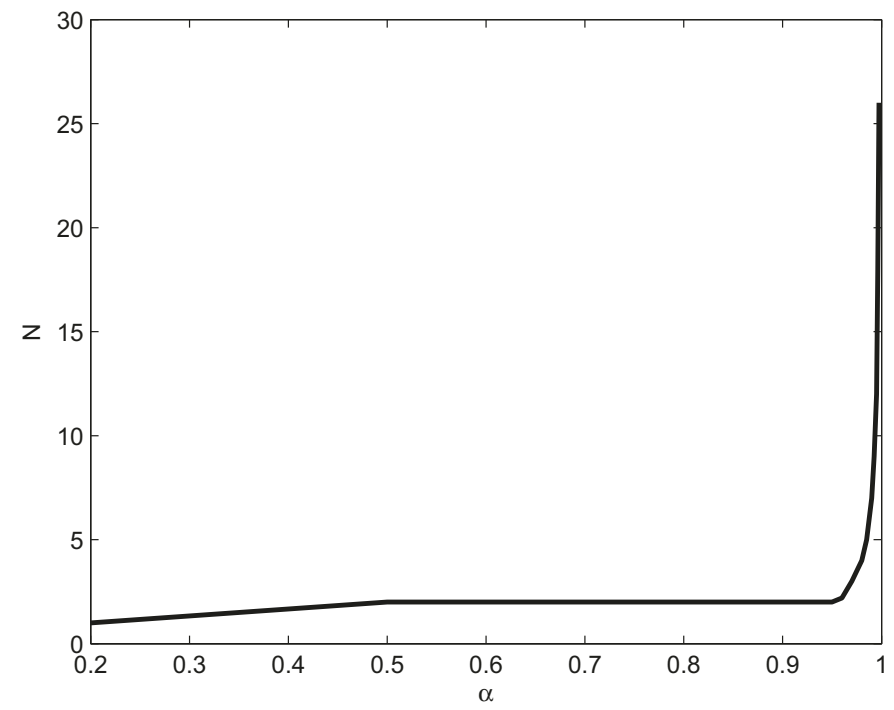

Figure 4.13: Truncation $N(\alpha)$ based on truncation parameter $c=1$.

\subsection{New Damped Model Problem}

In order to understand the transition between the two regimes that we discussed in Chapter 3, we introduce a new damping model problem with different boundary conditions from the previous problem in Section 4.1 to explain how the qualitative nature of the response depends on the underlying problem features, in particular the boundary conditions. 


\subsubsection{The Model Formulation}

Consider the following nonlinear damping model problem

$$
\begin{aligned}
& u_{\theta}=v_{x}, \\
& v_{\theta}=\frac{1}{\lambda^{2}}\left(u_{x}-\frac{u^{2}}{2}+\lambda^{2} \nu \epsilon^{2} v_{x x}\right),
\end{aligned}
$$

where $\nu$ is the damping coefficient, and as before of order $O(1)$.

On eliminating $v(x, \theta)$ from equations (4.91) and (4.92), we get the same damped nonlinear model problem as in equation (4.1).

Based on the context of acoustic problems, we now assume the boundary conditions are given by

$$
u(0, \theta)=\epsilon^{3} \sin (\theta), \alpha v(1, \theta)+(\alpha-1) v_{x}(1, \theta)=0,
$$

where $\alpha$ is arbitrary parameter and $\epsilon^{3}$ the forcing amplitude.

\subsubsection{Analytic Approximation}

To obtain approximate solution of the damped nonlinear model problem given in equations (4.91) and (4.92) with the boundary conditions in (4.93), we carry out a weakly nonlinear analysis by using the perturbation method.

\section{Linear Theory}

The solution is written as a perturbation series in powers of $\epsilon$. We consider the 
following perturbation expansions

$$
\begin{aligned}
u(x, \theta) & =\epsilon u_{1}(x, \theta)+\epsilon^{2} u_{2}(x, \theta)+\epsilon^{3} u_{3}(x, \theta)+\cdots, \\
v(x, \theta) & =\epsilon v_{1}(x, \theta)+\epsilon^{2} v_{2}(x, \theta)+\epsilon^{3} v_{3}(x, \theta)+\cdots, \\
\lambda & =\lambda_{0}+\epsilon^{2} \delta+\cdots,
\end{aligned}
$$

where $u_{i}$ and $v_{i}$ are strictly order unity for each $i \geq 1, \lambda_{0}$ is the fundamental frequency and $\delta=\lambda_{2}$.

The assumption in (4.94)-(4.96) is that the output is $O(\epsilon)$ while the input is $O\left(\epsilon^{3}\right)$. We substitute the expansions (4.94)-(4.96) into equations (4.91)-(4.93) and equate terms of $O(\epsilon)$. The linear problem for $u_{1}(x, \theta)$ and $v_{1}(x, \theta)$ is given by:

$$
\begin{gathered}
u_{1 \theta}-v_{1 x}=0, \\
\lambda_{0}^{2} v_{1 \theta}-u_{1 x}=0,
\end{gathered}
$$

and

$$
u_{1}(0, \theta)=0, \alpha v_{1}(1, \theta)=(1-\alpha) v_{1 x}(1, \theta)
$$

By eliminating $u_{1}(x, \theta)$ from equations (4.97) and (4.98), $v_{1}(x, \theta)$ satisfies

$$
\lambda_{0}^{2} v_{1 \theta \theta}-v_{1 x x}=0
$$

Using equation (4.97), the forced boundary condition in (4.99) becomes

$$
v_{1 x}(0, \theta)=0
$$

Following the same arguments as we did in Section 4.1, we suppose the form of leading-order is

$$
v_{1}(x, \theta)=\phi(x)(A \sin (\theta)+B \cos (\theta))
$$


where $A$ and $B$ are arbitrary amplitudes at this order to be determined later when we obtain the solvability conditions at $O\left(\epsilon^{3}\right)$. After substituting this form into equation (4.100), we find that the eigenfunction $\phi(x)$ satisfies the following eigenvalue problem:

$$
\frac{d^{2} \phi(x)}{d x^{2}}+\lambda_{0}^{2} \phi(x)=0
$$

The boundary conditions are now given by

$$
\phi^{\prime}(0)=0, \quad \alpha \phi(1)+(\alpha-1) \phi^{\prime}(1)=0 .
$$

The solution for $\phi(x)$ is given by

$$
\phi(x)=C_{1} \sin \left(\lambda_{0} x\right)+C_{2} \cos \left(\lambda_{0} x\right) .
$$

The boundary conditions in (4.104) imply that $\lambda_{0}$ is determined by the eigenvalue equation

$$
(\alpha-1) \sin \left(\lambda_{0}\right)=\frac{\alpha \cos \left(\lambda_{0}\right)}{\lambda_{0}}
$$

Therefore the leading-order solution for $v_{1}$ is given by

$$
v_{1}(x, \theta)=\cos \left(\lambda_{0} x\right)[A \sin (\theta)+B \cos (\theta)] .
$$

Using $u_{1 \theta}=v_{1 x}$, the leading-order for $u_{1}$ is

$$
u_{1}(x, \theta)=\lambda_{0} \sin \left(\lambda_{0} x\right)(A \cos (\theta)-B \sin (\theta)) .
$$

Next, in order to obtain a complete linear solution, it only remains to obtain amplitudes, and this requires computing the nonlinear terms up to the third order. 


\section{Calculation of Amplitudes $A$ and $B$}

The first correction to linear theory in the perturbation solution (i.e., at $O\left(\epsilon^{2}\right)$ ) is given by

$$
\begin{aligned}
u_{2 \theta}-v_{2 x} & =0, \\
\lambda_{0}^{2} v_{2 \theta}+\frac{u_{1}^{2}}{2}-u_{2 x} & =0,
\end{aligned}
$$

with

$$
v_{2 x}(0, \theta)=0, \alpha v_{2}(1, \theta)=(1-\alpha) v_{2 x}(1, \theta) .
$$

The two equations (4.109) and (4.110) can be combined into a single nonlinear equation, by eliminating $u_{2}$ :

$$
\lambda_{0}^{2} v_{2 \theta \theta}-v_{2 x x}=-u_{1} u_{1 \theta}
$$

Using the leading-order of $u_{1}$ in (4.108), it clear that the form of $v_{2}(x, \theta)$ should be

$$
v_{2}(x, \theta)=C_{4}(x) \cos (2 \theta)+C_{5}(x) \sin (2 \theta) .
$$

Substituting this form of $v_{2}(x, \theta)$ into equation (4.112), then equating the coefficients of $\cos (2 \theta)$ and $\sin (2 \theta)$ respectively, we find that $C_{4}(x)$ and $C_{5}(x)$ satisfy the following equations:

$$
\begin{aligned}
& \frac{d^{2} C_{4}(x)}{d x^{2}}+4 \lambda_{0}^{2} C_{4}(x)=\frac{1}{2} \lambda_{0}^{2} A B \cos \left(2 \lambda_{0} x\right)-\frac{1}{2} \lambda_{0}^{2} A B \\
& \frac{d^{2} C_{5}(x)}{d x^{2}}+4 \lambda_{0}^{2} C_{5}(x)=\frac{\lambda_{0}^{2}}{4}\left(A^{2}-B^{2}\right)+\frac{\lambda_{0}^{2}}{4}\left(A^{2}-B^{2}\right) \cos \left(2 \lambda_{0} x\right)
\end{aligned}
$$

where the boundary conditions are

$$
C_{i}^{\prime}(0)=0, \alpha C_{i}(1)=(1-\alpha) C_{i}^{\prime}(1) i=4,5
$$


We should notice that the solutions of the functions $C_{4}(x)$ and $C_{5}(x)$ can be determined provided $2 \lambda_{0}$ is not an eigenvalue.

After solving each equation at (4.114) with corresponding boundary condition, we find the full expression of $v_{2}(x, \theta)$ by substituting the solutions of $C_{4}(x)$ and $C_{5}(x)$ into equation (4.113). Next, $u_{2}(x, \theta)$ is calculated from equation (4.109).

The balance at $O\left(\epsilon^{3}\right)$ is of the form

$$
\begin{gathered}
u_{3 \theta}-v_{3 x}=0 \\
\lambda_{0}^{2} v_{3 \theta}-u_{3 x}+2 \lambda_{0} \delta v_{1 \theta}-\nu \lambda_{0}^{2} v_{1 x x}+u_{1} u_{2}=0,
\end{gathered}
$$

with

$$
v_{3 x}(0, \theta)=\cos (\theta), \alpha v_{3}(1, \theta)=(1-\alpha) v_{3 x}(1, \theta) .
$$

On eliminating $u_{3}$, and using equations (4.97), (4.109) and (4.116), the equation at $O\left(\epsilon^{3}\right)$ to determine $v_{3}$ is of the form

$$
\lambda_{0}^{2} v_{3 \theta \theta}-v_{3 x x}+2 \lambda_{0} \delta v_{1 \theta \theta}-\nu \lambda_{0}^{2} v_{1 x x \theta}+u_{1} v_{2 x}+u_{2} v_{1 x}=0 .
$$

Multiplying equation (4.119) by $\cos \left(\lambda_{0} x\right) \sin (\theta)$ and $\cos \left(\lambda_{0} x\right) \cos (\theta)$ respectively and integrating both sides for $0 \leq x \leq 1,0 \leq \theta \leq 2 \pi$, then using the boundary condition in equation (4.118), we obtain the restrictions for the amplitudes $A$ and $B$. Consider now the case $\alpha=0.95$. Then the corresponding eigenvalue can be determined by substituting $\alpha=0.95$ in the eigenvalue equation (4.106) and solving for $\lambda_{0}$. We find that $\lambda_{0}=1.657830119$. However, the solvability conditions for $A$ and $B$ are given by:

$$
\begin{array}{ll}
0.2376794039 A^{3}-4.936180883 \delta A-11.24556154 \nu B-0.07922646761 B^{2} A & =0, \\
-0.07922646810 B^{3}-4.936180884 \delta B+0.2376794030 B A^{2}+11.24556154 \nu A+\pi & =0 .
\end{array}
$$


Solving these equations with $\delta=0$ and $\nu=1$, the amplitudes $A$ and $B$ are

$$
A=-0.2793621, B=-0.0004608 .
$$

Following the numerical simulation approach discussed in Section 4.3, we carry out the numerical solution of the nonlinear wave model problem given by equations (4.91)(4.93). Figure 4.14 shows a comparison of the leading-order perturbation solution $v_{1}(x, \theta)$ using one mode with numerical solution for the case $\alpha=0.95$. Comparing Figure 4.14 with the corresponding Figure 4.2 for $\alpha=0.95$ where the boundary conditions are on $u$ only, we observe that now the form of the boundary conditions is playing a key role in the solvability conditions, and we obtain a very close agreement between numerical and analytical solutions.

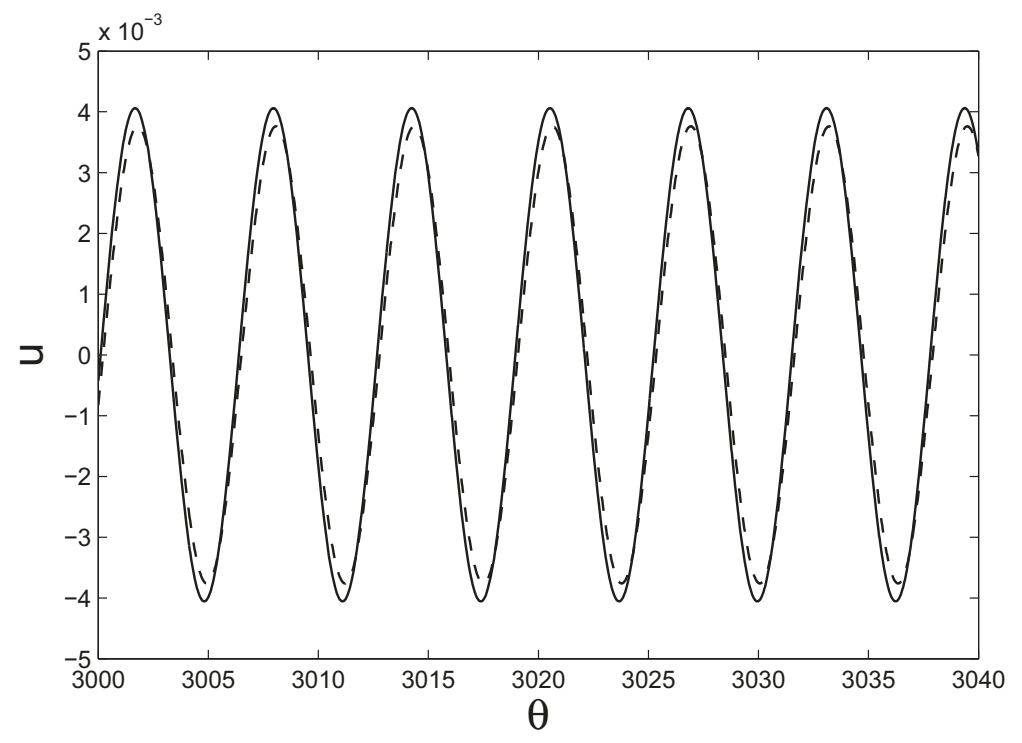

Figure 4.14: Comparison of numerical solution (solid line) to leading-order approximation (dashed line) $v 1(x, \theta)$ with $\alpha=0.95$.

Next, some conclusions are summarized, and some directions for future work are also presented in the coming chapter. 


\section{Chapter 5}

\section{Conclusions and Future Work}

The transition between the continuous and unbounded regimes, and how some features of the nonlinear wave system, such as nonlinearity, boundary conditions and damping play a crucial role in the qualitative nature of the response. Based on the related literature and motivated by observations in the context of acoustic wave systems, the primary goal was to achieve a better insight to understanding the transition between these two resonant-response outcomes and their correlation to the nature of the eigenvalue spectrum. These aspects were studied using various nonlinear wave model problems and served to explain how some features like nonlinearity of form, the nature of the spectrum and how the damping coefficients with many boundary conditions play a main effect in the outcome responses. We obtained an analytical approach, showing that, for some cases, higher modes must be included to fully capture the solution behavior.

Next, some conclusions are summarized, and some directions for future work are also presented in the coming two sections. 


\subsection{Conclusions}

First, we presented two different regimes for the response of acoustic wave systems. The first one is the continuous response as shown in Chapter 2. In this regime, we illustrated how the effect of geometry influenced the response. We introduced the example of an inhomogeneous gas between two concentric spheres and showed that it could lead to a continuous motion even at resonance. Also, we explained the correlation between the continuous response and incommensurate eigenvalues. Then we used a nonlinear geometric acoustic analysis method to estimate the analytical solution when the dominant first harmonic approximation is not valid. The approximation results obtained by this method are in good agreement with the numerical solution. Furthermore, these results support the fact that an unbounded response arises and is correlated with commensurate eigenvalues.

In Chapter 3, we considered some nonlinear model problems that achieve a better understanding of the nature of the transition between continuous and unbounded responses and how the connection between these two regimes has an effect on the outcomes. We used a Runge-Kutta method to carry out the numerical solution and then compared the result with the analytical solution. The nonlinear analysis of these examples was based on perturbation theory and depends on the parameter $\alpha$ in the boundary condition. We illustrated that, while the features of the spectrum are important to specify the type of response, as we clarified in Case 1 and Case 2 in Example 1, the form of the nonlinearity also plays a significant role in determining the nature of the output.

A damped nonlinear model problem was studied in Chapter 4, and an analytical solution for the linear and nonlinear theory was discussed. We investigated many cases of the model problem by varying the boundary condition parameter $\alpha$, providing a better understanding of the continuous transitions that connect and build upon the existing methodologies that are valid on either side. The key to this is how to reconcile 
the differences in scaling that underlies the forcing scale. In fact, in the case $\alpha=1$, we obtain the analytical approximate solutions with the forcing of order $O\left(\epsilon^{3}\right)$ where multiple modes must be included to account for the resonant nonlinear effects and achieve good agreement with numerical simulation. However, for the case $\alpha=0$, we obtain the analytical approximate solutions with forcing of order $O\left(\epsilon^{2}\right)$. In most cases, the approximate analytical solutions are compared with numerical simulation.

In conclusion, the asymptotic approach to nonlinear resonant effects and the model problems provide good insight for understanding the transition between the continuous and unbounded responses, and their correlation to the underlying type of spectrum if it is commensurate or incommensurate. Moreover, we discussed how the response depends on the nonlinearity form, the nature of eigenvalue spectrum and the damping coefficient with different boundary conditions. These new insights will help understand more relevant in acoustic wave regime, and this will contribute to understanding when the solution of nonlinear wave equations would lead to an unbounded solution. In fact, as we have seen, the fixed boundary conditions in (4.2) depend on varying $\alpha$, and this is related to the context of a gas in the tube. For example, in the case $\alpha=1$, we have the same boundary condition where the end of the tube is open. However, when $\alpha=0$, we get the boundary corresponding to the case where the end of the tube is closed.

\section{$5.2 \quad$ Future Directions}

In this section, we discuss potential further extensions of the present study that arise from the work in this thesis. The primary future directions are as follows. 


\section{Analytical and Numerical Studies of Further Nonlinear Wave Model Prob- lems}

Based on the damped nonlinear model problems discussed in Chapter 4, we focued on the model problem where the nonlinearity term was $f(u)=u u_{x}$. We obtained the approximate analytic solutions for many cases and compared with numerical simulation results and saw good agreement, which provided a better understanding of the transition regime. However, we showed in Chapter 3 the effect of the nonlinearity form and the spectrum in the nature of the outcome. Thus there is a substantial effect of the nonlinearity form in the outcome responses. It would be interesting to consider another form of nonlinearity like $f(u)=u^{3} u_{x}$ or higher in the damped model problem in Chapter 4.

However, some difficulties may arise when going to higher order, both in terms of the asymptotic analysis (a must be continued to higher order) and in terms of the numerical computation (transient equilibration process will occur over a longer timescale).

\section{Extend and Generalize the Problem}

Once the role of a general nonlinear form in the damped model has been achieved, and an appropriate analytic approximation for solutions for the general form of the nonlinearity $u^{n} u_{x}$ can be found, then it will be interesting to extend and generalize to more physically relevant systems such as the equation for acoustic wave propagation in bounded media. This in turn will provide new insights into applications such as the design and manufacture of pumps, compressors, etc.

\section{Higher Orders and Higher Dimensions}

In this work, we studied the analytical and numerical approaches to second-order nonlinear wave model problems. Also, we discussed in Chapter 3 how the outcome responses are depending on the form of nonlinearity. Thus we would like to continue the work on studying higher-order nonlinear wave model problems and extend the 
focus to higher dimensions.

\section{Development of a New Approach}

The analytical approach developed and applied in this study, based on perturbation techniques, is one where the leading order solution contains a single mode or multiple modes. It would be interesting to determine the approximate solution by another method like the Tan-Cot method (see Kumar [34] ) and then compare with the analytical solutions found in this thesis. Moreover, to help understand the asymptotic solution with various nonlinearity term (i.e., $f(u)$ ) of the model problem discussed in Chapter 3 and 4, we may apply Kevorkian Energy method. For details we refer to [29]. 


\section{Appendix A}

\section{The Fredholm Alternative \\ Theorem}

In this appendix, we will study the Fredholm Alternative Theorem. Which was used in Chapter 4 to derive the solvability conditions of the amplitudes. Ivar Fredholm studied the existence and uniqueness for a given equation (operator) $L u=f$.

The Fredholm Alternative Theorem includes two parts: first, the equation has exactly one solution for all $f$. Secondly, the equation has many solutions for some $f$ and none for the others. Thus, why it calls alternative. It could occur in many forms as a statement for the solution to system of an algebraic equation, a theorem of integral equations or as a theorem for solutions of boundary-value problems. One of the most common forms is the Fredholm Alternative for the solvability of a linear equations. Before introducing the main theorem, we need to recall the definition of an adjoint operator.

Definition A.0.1. (adjoint operator) The adjoint operator $L^{*}$ of an operator $L$ in a Hilbert space $H$ is an operator such that $\langle L u, v\rangle=\left\langle u, L^{*} v\right\rangle$ for all $u, v$ in $H$.

The Fredholm Alternative Theorem is summarized in the following two theorems. 
Theorem A.0.1. (First Alternative) If $L$ is a bounded linear operator on a Hilbert space $H$, then $L u=f$ has a solution if and only if $\langle f, v\rangle=0$ for every $v$ such that $L^{*} v=0$.

Theorem A.0.2. (Second Alternative) A solution of $L u=f$, if it exists, is unique if and only if $u=0$ is the only solution of $L u=0$.

The second alternative is more familiar than the first one when we have a nonhomogeneous system of $n$ equations and $n$ unknowns. Then the solution for this system is unique if and only if the homogeneous problem has only the trivial solution.

A formulation in terms of boundary value problems is similar. However, in the adjoint operator definition, there is an additional term $S(u, v)$ as a result of some integrations by parts. So we have $\langle L u, v\rangle=S(u, v)+\left\langle u, L^{*} v\right\rangle$, where $S(u, v)$ involves the boundary conditions on $u$ and $v$.

Theorem A.0.3. The solution of the boundary-value problem $L u=f$, with boundary conditions $B u=g$, exists if and only if $\langle f, v\rangle-S(u, v)=0$ for all $v$ such that $L^{*} v=0$ and $B^{*} v=0$.

Example ( see [66] ) Consider the following differential equation

$$
u^{\prime \prime}+u=f(x)
$$

with the boundary conditions $u(0)-u(2 \pi)=\alpha$ and $u^{\prime}(0)-u^{\prime}(2 \pi)=\beta$, where $\alpha$ and $\beta$ are arbitrary constant.

We can write this in operator form, $L u=f$, with $L=\frac{d^{2}}{d x^{2}}+1$.

The classical solutions for homogeneous equation

$$
L v=0, \text { with } v(0)-v(2 \pi)=0 \text { and } v^{\prime}(0)-v^{\prime}(2 \pi)=0 \text {, }
$$


are given by a linear combination of $v=\sin (x)$ and $v=\cos (x)$. Subject to Theorem A.0.3, in order to study the existence of the solution of inhomogeneous case, we need to determine $S(u, v)$ :

$$
\begin{aligned}
S(u, v) & =\left[u^{\prime} v-u v^{\prime}\right]_{0}^{2 \pi} \\
& =u^{\prime}(2 \pi) v(2 \pi)-u(2 \pi) v^{\prime}(2 \pi)-u^{\prime}(0) v(0)+u(0) v^{\prime}(0)
\end{aligned}
$$

For $v=\sin (x)$, this yields

$$
S(u, \sin (x))=-u(2 \pi)+u(0)=\alpha .
$$

Similarly, $S(u, \cos (x))=\beta$.

The solvability conditions for existence of the solution in (A.1), subject to $<f, v>$ $-S(u, v)=0$, are given by

$$
\begin{aligned}
& \int_{0}^{2 \pi} f(x) \sin (x) d x=\alpha \\
& \int_{0}^{2 \pi} f(x) \cos (x) d x=\beta .
\end{aligned}
$$

If $L$ is a differential operator from the first alternative, we notice that, since the homogeneous case has a nontrivial solution, then an inhomogeneous case can have a solution if and only if the right side satisfies a appropriate orthogonality condition. 


\section{Bibliography}

[1] Bender, C. M., Orszag, S. A., 1999: Advanced mathematical methods for scientists and engineers: asymptotic methods and perturbation theory, Springer Science and Business Media, New York.

[2] Betchov. R. 1958: Nonlinear oscillations of a column of gas. Phys. Fluids, 1, 205-212.

[3] Bressan, A., 2000: Hyperbolic systems of conservation laws : the onedimensional cauchy problem. Oxford University Press, England.

[4] Caudrey, P.J., Eilbeck, J.C., Gibbon, J.D., 1975: The sine-Gordon equation as a model classical field theory. Il Nuovo Cimento B, 25(2), 497-512.

[5] Chu, B.T; Ying, P.I.,1963: Thermally driven nonlinear oscillations in a pipe with traveling shock waves. Phys. Fluids,6,1625-1637.

[6] Chester, W.,1964: Resonant oscillations in a closed tube. J. Fluid Mechanics, 18,44-64.

[7] Chester, W.,1981: Resonant oscillations of a gas in an open-ended tube. Proceedings of the Royal Society of London. Series A, 377(1771), 449-467.

[8] Chester, W., 1991: Acoustic resonance in spherically symmetric waves. Proceedings of the Royal Society of London. Series A, 434 (1891), 459 - 463. 
[9] Chester, W., Moser A., 1982 : On an equation which describes the resonant oscillations of a gas in an open-ended tube. Proceedings of the Royal Society of London. Series A, 380 (1779), 409-415.

[10] Chester, W., 1994: Nonlinear resonant oscillations of a gas in a tube of varying cross section. Proceedings of the Royal Society of London. Series A, 444(1922), 591-604.

[11] Chun, Y. D., Kim, Y. H., 2000: Numerical analysis for nonlinear resonant oscillations of gas in axisymmetric closed tubes. J. Acoust. Soc. Am., 108, $2765-2774$.

[12] Cox, E. A., Mortell, M. P., 1983: The Evolution of resonant oscillations in closed tubes. Journal of Applied Mathematics and Physics (ZAMP), 34(6), $845-866$.

[13] Cox, E. A., Mortell, M. P., 1989: The Evolution of resonant acoustic oscillations with damping. Proceedings of the Royal Irish Academy. Section A: Mathematical and Physical Sciences, 89A(2), 147-160.

[14] Cox, E. A., Mortell, M. P., Reck, S., 2002: Nonlinear standing and resonantly forced oscillations in a tube with slowly changing length. SIAM Journal on Applied Mathematics., 62(3) , 965-989.

[15] Amundsen, D.E., Mortell, M. P., Seymour, B.R, 2015: Resonant radial oscillations of an inhomogeneous gas in the frustum of a cone. Z. Angew. Math. Phys., 66, 2647-2663.

[16] Amundsen, D.E., Mortell, M. P., Seymour, B.R, 2017: Nonlinear resonant oscillations in open axisymmetric tubes: continuous solutions. Journal of Applied Mathematics and Physics (ZAMP). 
[17] Ellermeier,W., 1993: Nonlinear acoustics in nonuniform infinite and finite layers. J. Fluid Mech., 257, 183-200.

[18] Ellermeier,W., 1994a: Acoustic resonance of cylindrically symmetric waves. Proc. R. Soc. London, Ser. A, 445(1923), 181-191 .

[19] Ellermeier,W., 1997: Acoustic resonance of radially symmetric waves in a thermoviscous gas. Acta Mech., 121, 97-113.

[20] Debnath, L., 2005: Nonlinear partial differential equations for scientists and engineers. Birkhauser, Boston.

[21] Griffiths, G. W., Schiesser, W. E., 2012: Traveling wave analysis of partial differential equations : numerical and analytical methods with MATLAB and Maple. Academic Press.

[22] Gorkov, A.P., 1963: Nonlinear acoustic oscillations of a column of gas in a closed tube. Inzhenerno-fizicheski jurnal, 3, 246-250.

[23] Holmes, M. H., 1995: Introduction to perturbation methods. Springer Verlag, New York.

[24] Ilinskii, Y. A., Lipkens, B., Lucas, T. S., Van Doren, T. W., 1998: Nonlinear standing waves in an acoustic resonator. J. Acoust. Soc. Am., 104, 2664-2674.

[25] Jimenez,J., 1973: Nonlinear gas oscilliation in pipe. Part I. Theory, J.Fluid Mech., 59, 23- 46.

[26] Kaper, H. G., Garbey, M., 1991: Asymptotic analysis and the numerical solution of partial differential equations. M. Dekker, New York.

[27] Keller, J. J., 1977: Nonlinear acoustic resonances in shock tubes with varying cross-sectional area. ZAMP J. Appl. Math. and Appl. Phy., 28(1), 107-122. 
[28] Keller, J.B., Kogelman, S., 1973: Asymptotic theory of nonlinear wave propagation. SIAM J. Appl. Math., 24(3), 352-361.

[29] Kevorkian, J., 1972: A perturbation method for hyperbolic equations with small nonlinearities. SIAM J. Appl. Math., 22(2), 235-258.

[30] Kevorkian, J., 1990: Partial differential equations; analytical solution techniques.Chapman and Hall, London.

[31] Kevorkian, J., Cole, J. D., 1981: Perturbation methods in applied mathematics. Springer Verlag, New York.

[32] Kurihara, E., Yano, T., 2006 : Nonlinear analysis of periodic modulation in resonances of cylindrical and spherical acoustic standing waves. Phys. Fluids., 18, 117107.

[33] Kurihara,E., Inoue,Y., and Yano, T. , 2005: Nonlinear resonant oscillations and shock waves generated between two coaxial cylinders. Fluid Dyn. Res.,36, 45-60.

[34] Kumar, R., Kumar, M., Kumar, A., 2013: Some soliton solutions of nonLinear partial differential equations by Tan-Cot Method. IOSR Journal of Mathematics (IOSR-JM), 6(6), 23-28.

[35] Lawrenson, C.C., Lipkens, B., Lucas, T.S., Perkins, D.K. and Van Doren, T.W., 1998: Measurements of macrosonic standing waves in oscillating closed cavities. J. Acoust.Soc. America, 104(2), 623-636.

[36] Lettau, E., 1939: Messungen un schwingunger von gassaulen mit stellen fronten in rohrleitgunen. Deut Kraftfahrforsch, 39, 1-17.

[37] LeVeque, R. J., 1992: Numerical methods for conservation laws. Birkhauser Verlag Basel, Boston . 
[38] Randall, R. H., 1951: An introduction to acoustic. Addison Wesley Press, Cambridge.

[39] Russell, L.H., 2013: A course in mathematical methods for physicists. Boca Raton, New York.

[40] Mathews, J. H., Fink, K. D., 2004: Numerical methods using MATLAB. Upper Saddle River, N.J. Pearson.

[41] McOwen, R. C., 2003: Partial differential equations : methods and applications. Upper Saddle, N.J. : Prentice Hall.

[42] Mortell, M.P., Seymour, B.R., 2005: Finite amplitude, shockless, resonant vibrations of an inhomogeneous elastic panel. Math. Mech. Solids, 10(4), 427440.

[43] Mortell, M. P., Mulchrone, K. F., Seymour, B. R., 2009: The evolution of macrosonic standing waves in a resonator. Int. J. Eng. Sci., 47, 1305-1314.

[44] Mortell, M. P., Seymour, B.R, 2004: Nonlinear resonant oscillations in closed tubes of variable cross-section. J. Fluid Mech., 519, 183-199.

[45] Mortell, M. P. Seymour, B.R., 2007: Resonant oscillations of an inhomogeneous gas in a closed cylindrical tube. Discrete Cont. Dyn. Syst. Ser. B, A.I.M.S. 7(3), 619-628.

[46] Mortell, M. P. Seymour, B.R., 2010: The propagation of small amplitude nonlinear waves in a strongly inhomogeneous medium. Mathematics and Mechanics of Solids. 16(6): 637-651.

[47] Nayfeh, A. H., 1981: Introduction to perturbation techniques. Wiley, New York.

[48] Nayfeh, A. H., 1973: Perturbation methods. Wiley, New York. 
[49] Nayfeh, A. H., 1995: Applied nonlinear dynamics : analytical, computational, and experimental methods. Wiley, New York.

[50] Nayfeh, Ali H., Mood, Dean T., 1979 : Nonlinear oscillations. Wiley, New York.

[51] Ockendon, J. R., 2003: Applied partial differential equations. Oxford University Press, New York.

[52] Ockendon, H., Ockendon, J.R., 2001: Nonlinearity in fluid resonances. Meccanica, 36(3), 297-321.

[53] Ockendon, H., Ockendon, J.R., Peake, M. R., Chester,W. , 1993: Geometrical effects in resonant gas oscillations. J. Fluid Mech., 257, 201-217.

[54] Pierce, A. D., 1981: Acoustics an introduction to its physical principles and applications. McGraw-Hill Book Co., New York.

[55] Quarteroni, A., Sacco, R., Saleri, F., 2000: Numerical mathematics. Springer, New York.

[56] Seymour, B. R., Mortell, M. P., Amundsen, D. E., 2011: Resonant oscillations of an inhomogeneous gas between concentric spheres. Proceedings of the Royal Society of London. Series A, 467(2132), 2149-2167.

[57] Seymour, B. R., Mortell, M. P., Amundsen, D. E., 2012: Asymptotic solutions for shocked resonant acoustic oscillations between concentric spheres and coaxial cylinders. AIP Physics of Fluids,24(2), doi:10.1063/1.3687611.

[58] Seymour, B.R., Mortell, M. P., (1973 a): Resonant acoustic oscillations with damping: small rate theory. J. Fluid Mech., 58, 353-373.

[59] Seymour, B.R., Mortell, M. P., (1973 b): Nonlinear resonant oscillations in open tubes. J. Fluid Mech.,60 , 733-749. 
[60] Seymour, B.R., Mortell, M. P., (1975 b): Nonlinear geometrical acoustic. In Mechanics Today, 2 , 251-312.

[61] Seymour, B.R., Mortell, M.P., 1980 : A finite rate theory of resonance in a closed tube: discontinuous solutions of a functional equation. J. Fluid Mechanics., 99, 365-382.

[62] Smoller, J., 1983: Nonlinear partial differential equations. American Mathematical Society, 2nd. ed.

[63] Varley, E., Cumberbatch, E., 1970: Large amplitude waves in stratified media: acoustic pulses, J. Fluid Mech., 43, 513.

[64] Von Mises, R., Friedrichs, K. O., 1971: Fluid dynamics. Springer Verlag, New York.

[65] Waltman, P., 2004: A second course in elementary differential equations. Academic Press, Orlando.

[66] White, R.B., 2005: Asymptotic analysis of differential equations. Imperial College Press, London.

[67] Whitham, G.B., 1974: Linear and Nonlinear waves. Wiley Interscience, New York.

[68] Whitham, G.B., 1953: The propagation of weak spherical shocks in stars. Comm. Pure Applied Math, 6, 397-414.

[69] Wazwaz, A. M., 2005: The tanh and the sine-cosine methods for compact and noncompact solutions of the nonlinear KleinâGordon equation. Applied Mathematics and Computation, 167, 1179-1195. 